\title{
29. PETROLOGY, MINERALOGY, AND CHEMISTRY OF BASALTIC ROCKS: LEG 81 ${ }^{1}$
}

\author{
R. K. Harrison and R. J. Merriman, British Geological Survey, \\ with contributions by \\ Jane Evans, Dawn Hutchison, A. E. Davis, K. A. Holmes, P. Joseph, \\ V. A. Judge, and C. W. Wheatley, British Geological Survey²
}

\begin{abstract}
We detail the petrography and mineralogy of 145 basaltic rocks from the top, middle, and base of flow units identified on shipboard along with associated pyroclastic samples. Our account includes representative electron microprobe analyses of primary and secondary minerals; 28 whole-rock major-oxide analyses; 135 whole-rock analyses each for 21 trace elements; 7 whole-rock rare-earth analyses; and 77 whole-rock X-ray-diffraction analyses.

These data show generally similar petrography, mineralogy, and chemistry for the basalts from all four sites; they are typically subalkaline and consanguineous with limited evolution along the tholeite trend. Limited fractionation is indicated by immobile trace elements; some xenocrystic incorporation from more basic material also occurred. Secondary alteration products indicate early subaerial weathering followed by prolonged interaction with seawater, most likely below $150^{\circ} \mathrm{C}$ at Holes 552, 553A, and 554A. At Hole 555, greenschist alteration affected the deepest rocks (olivine-dolerite) penetrated, at $250-300^{\circ} \mathrm{C}$.
\end{abstract}

\section{INTRODUCTION}

Deep Sea Drilling Project (DSDP) Leg 81 drilled four sites transecting the rifted "passive" margin of the Rockall microcontinent, with the object of studying the sedimentary and igneous evolution of this type of margin (Fig. 1). Of particular interest are the age and nature of the westward-dipping reflectors, which dominate shallow seismic records of the Rockall type of margin (Roberts et al., this volume). Leg 81 located Sites 552 and 553 at the midpoint of the transect, and both bottomed in basaltic rocks above or within the upper dipping reflectors after passing through Paleocene and Tertiary successions. In Hole 552, $31.3 \mathrm{~m}$ of basalts were encountered and in Hole 553A, $183.0 \mathrm{~m}$ of basalts, the lowest $71.0 \mathrm{~m}$ corresponding to dipping reflectors.

Site 554 was located oceanward of these two sites on the crest of the "outer high," separating the zone of dipping reflectors from the oceanic crust to the west. The outer high lies at the edge of oceanic magnetic Anomaly 24B ( 52 m.y. in age, corresponding to the initiation of rifting-spreading between Greenland and Rockall). Hole 554A bottomed in $72 \mathrm{~m}$ of probable pillow basalts of normal and reverse polarity underlying Pleistocene and Tertiary sediments. The basement of the "outer high" here is formed of overlapping normal-polarity basalts which may represent the first-formed oceanic crust. Site 555 was located midway between Hatton and Edoras banks, some $40 \mathrm{~km}$ east of the zone of dipping reflectors drilled at Site 553. The objective here was to compare the subsidence history of these banks with that

\footnotetext{
${ }^{1}$ Roberts, D, G., Schnitker, D., et al., Init. Repts. DSDP, 81: Washington (U.S. Govt. Printing Office).

2 Addresses: (Harrison, Merriman, Wheatley), British Geological Survey, Exhibition Road, London SW7 2DE, United Kingdom; (Evans, Hutchison, Davis, Holmes, Joseph, Judge) British Geological Survey, Gray's Inn Road, London, WCIX 8NG.
}

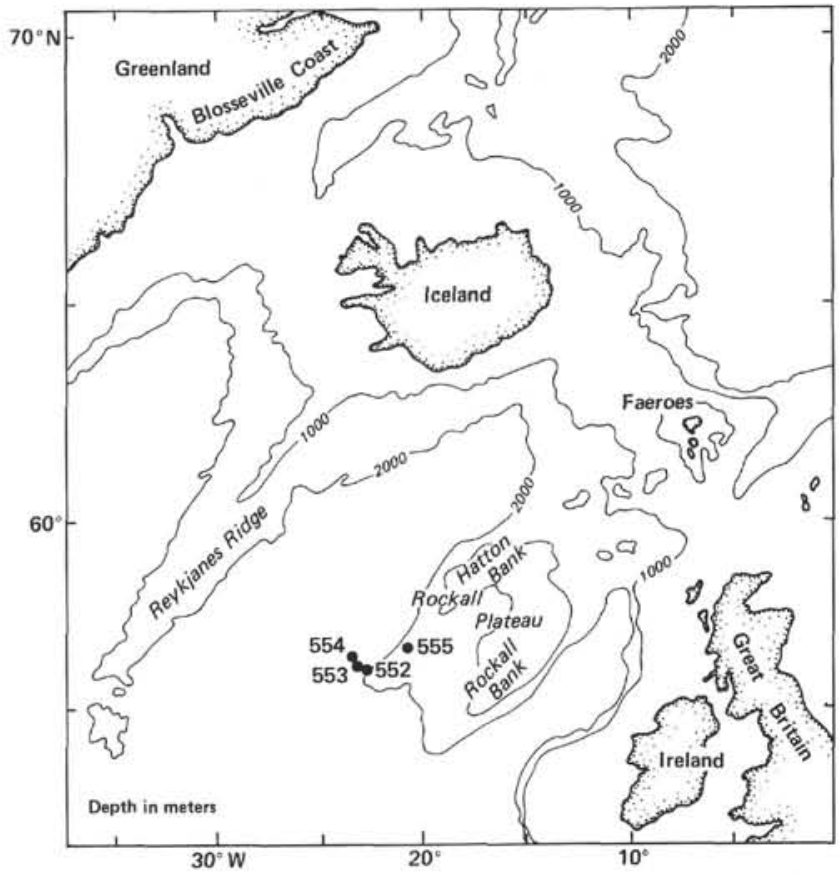

Figure 1. Map of the North Atlantic showing Leg 81 sites and other localities referred to in the text.

found at the deeper sites. After penetrating Pleistocene and Miocene sediments, a major hiatus was found between the early Eocene and Miocene. The early Eocene succession contained tuffs and sediments, the former increasing downward and passing into pillow basalts interbedded with autoclastic breccias. These volcanics may represent the dipping reflectors to the west. Late Paleocene marine mudstones underlie these rocks which rest in turn on about $294 \mathrm{~m}$ of massive basalt of reversed polarity (Roberts et al., this volume). 


\section{R. K. HARRISON ET AL.}

We record here the petrography and mineralogy of sampled basalts and associated rocks from the four sites. However, since the basalts are mainly microcrystalline and their primary mineralogy relatively uniform, we have also investigated their major and trace-element chemistry, mainly to determine any inter- or intrahole variations, to classify and characterize, and so to allow comparison with other DSDP basalt sites. For this, we have relied heavily on the very detailed shipboard logs (Roberts et al., this volume) to relate our (unavoidably restricted) samples to the flow units and sequences identified therein for each of the sites.

\section{PETROGRAPHY}

\section{METHODS}

We selected samples from near the top, middle, and base of individual lava flow units where these were identified in the shipboard log. We examined 4 sections from the restricted sequence of Hole 552; 83 thin-sectioned cores, at intervals ranging from $20 \mathrm{~cm}$ to $10 \mathrm{~m}$, from Hole 553A; 8 sections of the small thickness penetrated in Hole 554A basalts; and 51 sections from Hole 555 basalts and dolerites, at intervals from $50 \mathrm{~cm}$ to $15 \mathrm{~m}$. We also examined 19 thin-polished sections of cores selected for microprobe analyses of individual minerals, based on our initial petrographical examination. Because of alteration, especially to smectite, many specimens required special treatment for sectioning. From our examination of the thin sections, we selected 31 samples for X-ray diffraction analysis of secondary minerals. Twenty major oxide analyses were made by beta-probe of the main and leastaltered petrographical varieties, and we report analyses for 22 trace elements by direct reader spectrography of most of the sectioned samples; rare-earth elements of 7 samples were analyzed by neutron activation. We made 12 density determinations of the least-altered samples from Hole 555 cores, but because of the prevalence of vesicles, further determinations were not justified.

\section{Hole 552}

A basalt sample from Subunit Va, (Sample 552-21-3, $75-77 \mathrm{~cm})$ is dark greenish gray $(5 \mathrm{GY} 4 / 1)$, vesicular, aphyric, and heavily smectitized. Spheroidal vesicles ${ }^{3}$ (averaging $1 \mathrm{~mm}$ diameter) are lined or partly filled with fibroradial brown smectite and microframboidal pyrite. The texture is microphyric with common, randomly oriented labradorite laths (averaging $0.4 \times 0.04 \mathrm{~mm})\left(\mathrm{An}_{60}\right)$ set in a microcrystalline $(0.06 \times 0.006 \mathrm{~mm})$ groundmass. Clinopyroxene is colorless $(0.25 \mathrm{~mm}$ across $)$ and subhedral but is rarely unaltered. The turbid smectitic base is charged with opaque iron oxide. Microcrystalline felts of skeletal iron oxide in a devitrified glass base surround vesicles. Two basalt samples of Subunit Vb (Samples 552-22-1, 137-144 cm and 552-22-2, 35-38 cm) are dark gray (N3), contain abundant spheroidal to irregular vesicles, averaging $1.5 \mathrm{~mm}$ diameter, partly filled with fibroradial smectite. The first basalt also shows subspherical regions 1-2 mm across, of devitrified microcrystalline material charged with skeletal opaque ore, and resembling the mesostasis of tholeiitic dyke rocks. This material may also enclose smectite-filled vesicles which in turn are surrounded by tangentially arranged plagioclase laths.

The possibility that these represent an immiscible Ferich liquid was investigated by electron microprobe scans

\footnotetext{
${ }^{3}$ The term vesicle is used throughout and denotes cavities resulting from gas bubbles (vesicle: sensu stricto) and also those partly or wholly filled with minerals (amygdales).
}

across the quench-textured areas and compared with similar scans of adjacent microcrystalline lava. These analyses, however, reveal no significant differences between the contrasting textural portions, and it is concluded that the quenched "spheres" represent volatile-rich bubbles of liquid in a more viscous or partly solidified melt, which were quenched by sudden degassing. The texture is a random latticework of labradorite laths averaging $0.6 \times 0.06 \mathrm{~mm}$ with colorless anhedra of clinopyroxene about $0.4 \mathrm{~mm}$ across in a groundmass that is microcrystalline or devitrified glass (intersertal texture) altered to smectite with opaque dusty ore. Electron microprobe analyses show that plagioclase microphenocrysts have a composition $\mathrm{An}_{63}$, the clinopyroxene is augite $\mathrm{Mg}_{39} \mathrm{Fe}_{21} \mathrm{Ca}_{40}$, and the opaque is Ti magnetite. A second basalt sample from this subunit is coarse-grained, with labradorite laths $\left(\mathrm{An}_{57}\right)$ averaging about $0.65 \times 0.06 \mathrm{~mm}$ in partly subophitic intergrowth with colorless to pale green clinopyroxene $(0.3 \mathrm{~mm})$. Interstitial iron-titanium oxide and much devitrified, smectitized glassy mesostasis, some charged with opaque $\mathrm{Fe}-\mathrm{Ti}$ oxide needles, are present. Abundant smectite-lined vesicles interconnect and areas of quenchtextured basalt again suggest sudden degassing of the lava.

\section{Hole 553A}

\section{Textures and Lithologies}

Samples from the three subunits (Va,b,c) include: massive, homogeneous aphyric basalts, with much variation in vesicle shapes, size distribution, orientation, and mineral infilling; microphyric basalts; flow-brecciated basalts, vitroclastic tuffs, and possible agglomerates. Basalts (excluding vesicles) are mainly hues of gray, bluish gray, or more commonly greenish gray. Vitroclastic tuffs in Subunit $\mathrm{Vb}$, in contrast, are dusky red $(10 \mathrm{R} 2 / 2)$ to blackish red (near 5R/2/2); scoriaceous tops of certain flows (e.g., Sample 553A-52-3, 8-11 cm) may be of similar color, and autobrecciated material within flows (e.g., Sample 553A-55-3, 71-74 cm) is pale purple as a result of ferric oxide.

The texture of the basalts irrespective of position within flow units is mainly trachytic and hyalopilitic with variable content of plagioclase microphenocrysts. Samples from near tops of flows may be aphyric or weakly to moderately microphyric with less than $10 \%$ volume microphenocrysts. In the former, plagioclase occurs as felts of needles or laths in the range $0.1 \times 0.01 \mathrm{~mm}$ to 0.02 $\times 0.001 \mathrm{~mm}$, with composition $\mathrm{An}_{57}$ to $\mathrm{An}_{60}$. There are coarser $(0.4 \times 0.06 \mathrm{~mm})$ and finer aphyric $(0.04 \times$ $0.002 \mathrm{~mm}$ ) patches (Sample 553A-46-4, 122-124 cm) and also microphyric associated with aphyric material giving a pseudobreccia appearance (Sample 553A-45-4, 16-20 $\mathrm{cm}$; Plate 1, Fig. 3). In the microphyric or glomerophyric examples, plagioclase laths range from $0.3 \times 0.06 \mathrm{~mm}$ $\left(\mathrm{An}_{52}\right)$, to $3.0 \times 1.8 \mathrm{~mm}\left(\mathrm{An}_{60}\right)$ but are mainly about $1.54 \times 0.8 \mathrm{~mm}$. The groundmass plagioclase laths average about $0.04 \times 0.003 \mathrm{~mm}$ across $\left(\mathrm{An}_{57}\right.$ to $\left.\mathrm{An}_{60}\right)$. Ferromagnesian and accessory minerals are mainly dispersed as minute anhedral, interstitial smectitized grains seldom exceeding $0.03 \mathrm{~mm}$. 
Samples from within flows differ to some extent from those from near the tops, being predominantly plagioclase microphyric or glomerophyric (as in Subunit Vc, Samples 553A-51-1, 54-57 cm and 553A-59-4, 93-95 cm: see Plate 1, Figs. 5 and 6, respectively). Clinopyroxene rarely occurs in the microphenocrystic clusters, but more generally interstitially in the groundmass. Plagioclase microphenocrysts range from $0.2 \times 0.06 \mathrm{~mm}$ toward the top of the sequence in Subunit Vc to $3.0 \times 2.0 \mathrm{~mm}$ in feldspar-phyric samples near the base (Samples 553A$56-3,91-94 \mathrm{~cm}$ and 553A-59-4, 93-95 cm). The groundmass feldspar laths do not co-vary in size with the microphenocrysts. Thus in the feldspar-phyric samples the groundmass needles average only $0.06 \times 0.01 \mathrm{~mm}$, and these sizes are also common in the feldspar-microphyric varieties. The midflow samples also differ from those near tops in generally containing less-altered clinopyroxene, which is nearly always interstitial to the groundmass feldspar and averages about $0.02 \mathrm{~mm}$.

Samples from near the base of flows range from aphyric to feldspar-phyric (e.g., in Subunit Vb, Sample 553A47-4, 81-84 cm, Plate 1, Fig. 4). In aphyric types, felted plagioclase needles average $0.06 \times 0.002 \mathrm{~mm}$ with intergranular euhedral clinopyroxene averaging $0.01 \mathrm{~mm}$. Phyric and microphyric types have phenocrystic plagioclase laths ranging from $3.0 \times 2.0$ to $0.5 \times 0.3 \mathrm{~mm}$, the groundmass being very fine grained to the limits of optical resolution. Clinopyroxene is interstitial, anhedral, and very fine grained $(0.01-0.02 \mathrm{~mm})$.

In general, therefore, samples near the tops and bases of flows tend to be aphyric (although phyric near some bases) and plagioclase-microphyric or plagioclase-phyric to glomerophyric elsewhere. Clinopyroxene and accessories are mainly interstitial in the groundmass and only rarely form microphenocrysts. There does not appear to be any direct relationship between size, shape, and abundance of vesicles and the textures of the basalts.

\section{Mineral Composition}

The least-altered basalts indicate a simple assemblage of primary minerals: essential plagioclase and clinopyroxene as microphenocrysts (where present) and forming the groundmass, with accessory opaque ore, principally magnetite, and variable proportions of devitrified glassy residuum. Mineralogically the basalts are "tholeiitic," although the use of this term in the context of ocean-floor basalts has been criticized (e.g., Cann, 1971). Rare (pseudomorphed) olivine was observed in only three samples in Subunits Va and Vb (Samples 553A-40-1, $79-82 \mathrm{~cm}$; 553A-46-4, 122-124 cm; and 553A-48-6, 122$125 \mathrm{~cm}$ ). A trace of possible orthopyroxene occurs in Sample 553A-46-1, 121-125 cm. There is no discernible variation in mineral composition or proportions with sample depth or with position in a flow unit. We modally analyzed a selection of the least-altered basalts by point counter (see Table 3 ), and these included the main textural and mineralogical variants. In the phyric samples, phenocrysts range from 1 to $5 \%$ (sparsely to moderately phyric) and are principally plagioclase and rarely augite. In the aphyric varieties, and in the groundmass of the phyric types, clinopyroxene generally exceeds pla- gioclase: plagioclase (32-41\% volume augite $41-51 \%$ ). Titaniferous magnetite and other opaques are mainly minor $(4-16 \%)$. The remainder of the rock is mainly secondary smectite, but it is not possible to attribute this specifically to replaced primary ferromagnesians or to altered glassy mesostasis. An averaged composition of the freshest basalts is approximately plagioclase (principally labradorite), $36 \%$ volume; clinopyroxene, $48 \%$; titaniferous magnetite, $8 \%$; and smectite plus other secondaries, $8 \%$. Olivine is absent or sparse, as in Samples $553 \mathrm{~A}-40-1,70-82 \mathrm{~cm}$; 553A-46-4, 122-124 cm; and $553 \mathrm{~A}-48-6,122-125 \mathrm{~cm}$. A trace of orthopyroxene occurs in Sample 553A-46-1, 121-125 cm.

There is no discernible variation in modal composition or in mineral composition with sample depth in the hole, or in the flow units. We summarize primary mineral data in Table 1, with many optical determinations of the plagioclase composition, together with precise electron microprobe analyses of plagioclase microphenocrysts, groundmass plagioclase, clinopyroxene, and opaque oxides. The average microphenocryst plagioclase composition on optical data is $\mathrm{An}_{60}$ (range $\mathrm{An}_{51}-\mathrm{An}_{86}$ : labradorite to bytownite), groundmass plagioclase $\mathrm{An}_{56}$ (range $\mathrm{An}_{30}-\mathrm{An}_{70}$ : andesine to labradorite). Microprobe analyses show that microphenocrysts in feldspar-phyric basalts are more anorthite rich than co-existing groundmass plagioclase, the former reaching $\mathrm{An}_{86}$ toward the base of the succession. A consistent disparity between groundmass and microphenocryst composition (see Table 6) combined with a lack of zonation and marginal resorption of some plagioclase microphenocrysts suggests that the latter are fractionated xenocrysts.

Of six samples analyzed for pyroxene in Units $\mathrm{Vb}$ and $\mathrm{Vc}$, five differ little within the augite range of $\mathrm{Mg}(42-$ 48) : $\mathrm{Fe}(18-25): \mathrm{Ca}(33-36)$ (mean $\mathrm{Mg} 46: \mathrm{Fe} 20: \mathrm{Ca} 34)$ and indicate little variation in magma composition. Sample 553A-46-1, 121-125 cm contains both augite ( $\mathrm{Mg} 42$ : $\mathrm{Fe} 25: \mathrm{Ca} 33)$ and $\mathrm{Mg}$-pigeonite ( $\mathrm{Mg} \mathrm{52:Fe} \mathrm{34:Ca} \mathrm{14)} \mathrm{in-}$ dicating a tholeiitic affinity. The principal opaque phase is titanium-magnetite disseminated as irregular grains in the groundmass.

\section{Vesicles and Secondary Minerals}

Flow tops are mainly highly amygdaloidal and variably filled with smectite, celadonite, or less commonly, silica. Vesicles range up to $1 \mathrm{~cm}$ across but may extend to give a pseudobreccia texture; some may be fluxioned. Vesicles may also be sparse near flow tops; they show similar variation toward flow centers, ranging up to $2 \mathrm{~cm}$ in length. Ellipsoidal-spheroidal examples range up to about $7 \mathrm{~mm}$ across. Toward flow bases, vesicles are mainly dispersed and less than $3 \mathrm{~mm}$ diameter, but exceptionally (for example, in Sample 553A-56-1, 3-6 cm) irregular vesicles and vugs up to $1 \mathrm{~cm}$ across are common, indicating gas phases trapped beneath the lava flow. Thin sections show that smectite and celadonite are the most common fillings of vesicles throughout the sampled succession. Where both smectite and celadonite occur in a vesicle, smectite generally forms the radially microfibrous or cryptocrystalline brown rim and celadonite a radially fibrous or fibrous aggregate pleochroic 
Table 1. Summary of electron microprobe, optical, and X-ray diffraction analyses of basalts and associated rocks, Holes 552 and 553A.

\begin{tabular}{|c|c|c|c|c|c|c|}
\hline \multirow[b]{3}{*}{$\begin{array}{c}\text { Sample } \\
\text { (interval in } \mathrm{cm} \text { ) }\end{array}$} & \multicolumn{5}{|c|}{$\begin{array}{c}\text { Primary minerals }{ }^{\mathrm{a}} \\
\text { Optical and electron microprobe analyses }\end{array}$} & \multirow{3}{*}{$\begin{array}{c}\text { Secondary minerals }{ }^{\mathrm{b}} \\
\text { X-ray diffraction analyses }\end{array}$} \\
\hline & \multicolumn{2}{|c|}{ Plagioclase $(\% \mathrm{An})$} & \multirow[b]{2}{*}{$\begin{array}{l}\text { Pyroxene } \\
\mathrm{Mg}: \mathrm{Fe}: \mathrm{Ca}\end{array}$} & \multirow[b]{2}{*}{$\begin{array}{c}\text { Olivine } \\
\text { (alteration) }\end{array}$} & \multirow[b]{2}{*}{ Opaques } & \\
\hline & $\begin{array}{l}\text { Ground- } \\
\text { mass }\end{array}$ & $\begin{array}{l}\text { Microphe- } \\
\text { nocrysts }\end{array}$ & & & & \\
\hline \multicolumn{7}{|l|}{ Hole 552} \\
\hline $22-1,137-141$ & 41 & 63 & Aug. $39: 21: 40$ & & Ti-mag. & (sap.) \\
\hline \multicolumn{7}{|l|}{ Hole 553} \\
\hline $\begin{array}{l}38-1,62-65 \\
38-2,58-59 \\
39-1,2-4 \\
40-1,9-12 \\
40-1,79-82 \\
40-4,80-83 \\
42-2,42-46 \\
43-1,20-23 \\
43-2,111-114 \\
44-2,48-51\end{array}$ & $\begin{array}{c}(30) \\
(54) \\
50-62 \\
(54) \\
(57) \\
(54) \\
(54 \\
70 \\
(57) \\
(54)\end{array}$ & $\begin{array}{l}- \\
-\overline{82} \\
- \\
(54) \\
(54) \\
(57) \\
76 \\
(57) \\
(57)\end{array}$ & & $\begin{array}{l}\text { sap.-ps. } \\
\text { sap.-ps. }\end{array}$ & Ti-mag. & $\begin{array}{l}\text { Na-sap.>Ca-sap., cel., cal. } \\
\text { Na-sap.>cel. } \\
\text { (sap.>cel.) } \\
\text { Na-sap. cel. }\end{array}$ \\
\hline \multicolumn{7}{|l|}{ Subunit $\mathrm{Vb}$} \\
\hline $45-1,44-47$ & (tuff) & $\begin{array}{l}76 \text { (in } \\
\text { glass) }\end{array}$ & Aug. 48:18:34 & & & (sap., cel.) \\
\hline $45-1,87-91$ & $\begin{array}{l}68 \text { (in } \\
\text { glass) }\end{array}$ & & & & & Na-sap. > Ca-sap. cel., hem. (tr) \\
\hline $45-2,114-118$ & $(50)$ & $(54)$ & & & & Na-sap. $>$ cel. \\
\hline $45-3,79-83$ & (54) & (54) & & & & Na-sap. >cel. \\
\hline $45-5,17-19$ & (54) & (54) & & & & Ca-sap.> Na-sap., cel. \\
\hline $46-1,121-125$ & 64 & - & $\begin{array}{l}\text { Aug. } 42: 25: 33 \\
\text { Mg-pig. } 52: 34: 14\end{array}$ & & Ti-mag. & Na-sap. \\
\hline $46-3,56-59$ & (54) & (54) & & & & (sap.) \\
\hline $46-4,53-56$ & - & - & & & & (sap.) \\
\hline $46-4,122-124$ & - & $(52)$ & & sap.-ps. & & Na-sap. $>$ cel. \\
\hline $46-6,33-35$ & $(51)$ & (51) & & sap.-ps. & & (sap. >cel.) \\
\hline $47-3,73-76$ & - & (52) & & & & (sap. > cel.) \\
\hline $47-4,81-84$ & $(30-59)$ & (60) & & & & Na-sap. > cel. \\
\hline $47-4,128-130$ & - & - & & & & Na-sap. > cel. \\
\hline $48-1,13-16$ & - & (54) & & & & (sap. \cel.) \\
\hline $48-2,60-65$ & (54) & (54) & & & & Ca-sap. > Na-sap., cel. \\
\hline $48-3,97-101$ & 56 & (52) & & & & (sap.) \\
\hline $48-6,53-58$ & (57) & (57) & & & & (sap.) \\
\hline $48-6,74-76$ & (60) & (57) & & & & (sap. > cel.) \\
\hline $48-6,35-87$ & (Tuff) & & & & & Na-sap. $>$ cel. \\
\hline $48-6,122-125$ & $(60)$ & (54) & & sap.-ps. & & (sap.) \\
\hline $50-1,67-71$ & (57) & - & & & & Na-sap. > cel. \\
\hline $50-2,20-22$ & (60) & - & & & & Na-sap. $>$ cel. \\
\hline $50-3,32-35$ & (59) & - & & & & (sap.) \\
\hline $50-3,71-75$ & - & - & & & & Na-sap. > cel. \\
\hline \multicolumn{7}{|l|}{ Subunit $V_{c}$} \\
\hline $51-1,54-57$ & 68 & 82 & Aug. 46:20:34 & & & Ca-sap., nont., cal. \\
\hline $51-2,77-80$ & (57) & - & & & & (sap.) \\
\hline $52-1,78-82$ & (57) & - & & & & (sap.>cel.) \\
\hline $52-3,8-11$ & - & - & & & & Na-sap. >cel. \\
\hline $52-3,140-143$ & - & - & & & & Na-sap. > Ca-sap., cel. \\
\hline $53-1,77-80$ & $(56)$ & (57) & & & & \\
\hline $53-2,85-88$ & (56) & - & & & & \\
\hline $53-4,31-34$ & - & (57) & & & & Na-sap. >Ca-sap., cel. \\
\hline $53-4,76-79$ & (60) & (60) & & & & \\
\hline $54-1,64-67$ & - & (57) & & & & Na-sap. >Ca-sap., cel. \\
\hline $54-1,89-92$ & - & (57) & & & & \\
\hline $54-4,81-95$ & (57) & - & & & & \\
\hline $54-5,83-86$ & (57) & $(60)$ & & & & cel., Ca-sap. \\
\hline $55-1,36-38$ & - & - & & & & Na-sap.>Ca-sap., cel. \\
\hline $55-3,71-74$ & - & (57) & & & & \\
\hline $55-3,113-116$ & 65 & 77 & Aug. 47:17:36 & & Ti-mag. & cel., Ca-sap. \\
\hline $55-6,44-47$ & (57) & - & & & & \\
\hline $56-1,5-6$ & (58) & (57) & & & & \\
\hline $56-2,81-84$ & (54) & - & & & & \\
\hline $56-3,91-94$ & (54) & - & & & & \\
\hline $57-2,35-37$ & 67 & 86 & Aug. $47: 18: 35$ & & & \\
\hline $58-1,89-91$ & - & $(60)$ & & & & Na-sap., Ca-sap., cel. \\
\hline $59-2,117-120$ & - & $(60)$ & & & & \\
\hline $59-1,36-38$ & (58) & - & & & & Na-sap. > Ca-sap., cel. \\
\hline
\end{tabular}

Note: For list of symbols see Table 2.

aptical determination of anorthite content are shown in parentheses.

b Optical identification of secondary minerals are shown in parentheses. 
in hues of green, olive green, or blue green. In some samples (e.g., Sample 553A-38-2, 58-59 cm) the outer radially fibrous smectite appears to be continuous with the inner celadonite, but precise distinction optically is not possible. In some vesicles smectite alone occurs as radially fibrous or structureless masses and in others celadonite occurs alone as aggregates of microcrystalline fibers. Toward the lower part of the succession in Sample 553A-56-1, 5-6 cm, smectite and celadonite may be finely intercalated as vermiform radial aggregates. Chalcedony is sparse, but fills the cores of celadoniterimmed vesicles in Sample 553A-50-2, 20-22 cm. Calcite occurs sporadically in larger vesicles (as in Sample 553A$53-2,85-88 \mathrm{~cm}$ ) forming dendritic intergrowths with smectite. In some of the lowest samples celadonite appears to predominate over smectite.

\section{Fragmental Rocks}

The two samples of vitroclastic tuffs from Samples 553A-45-1, 44-47 cm and 553A-45-1, 87-91 cm (Subunit $\mathrm{Vb})$ are closely packed, poorly sorted aggregates of microlithic lava, pumice with spheroidal smectite-filled vesicles showing little compression, and abundant isotopic orange-red argillized glass shards (Plate 1, Fig. 2). Microphyric lava particles contain possible olivine pseudomorphs. The fine matrix is mainly comminuted, very angular orange-red glass particles averaging $0.08 \mathrm{~mm}$, and olive-green clay material. The color of the argillized glass results from dispersed hematite. A lithic tuff from Sample 553A-43-1, 62-66 cm (Subunit Va) shows basaltic fragments up to $1.5 \mathrm{~cm}$ across, set in a matrix of smectite and celadonite, which also fill vesicles. The basalts include microlithic aphyric patches with plagioclase needles (averaging $0.06 \times 0.003 \mathrm{~mm}$ ) and nests of coarser plagioclase $(0.4 \times 0.04 \mathrm{~mm})$, altered ferromagnesians, and disseminated ferric oxide. Some fragments are opaque as a result of intense iron oxide impregnation. The lava fragments appear to have fractured during flow, with later cementation by the clay minerals. Other samples (Samples 553A-45-2, 114-118 cm and 553A-45-4, 16-20 $\mathrm{cm}$, Subunit $\mathrm{Vb}$ ) are brecciated, with clasts of microphyric highly vesicular basalt set in a fine matrix of smectitic material veined by celadonite; they probably represent flow breccias (? cf. Aa-lavas). Sample 553A-52-3, $8-11 \mathrm{~cm}$ (flow top in Subunit Vc) is reddened, with aphyric, smectitic, hematitized basalt fragments in a fine clay matrix. Another (Sample 553A-54-1, 89-92 cm) probably owes its complex basalt textures to admixture of heterogeneous lava during flow and different cooling regimes, with no distinct clasts.

\section{Hole 554A}

\section{Textures and Lithologies}

Samples of basalt from Unit V are light gray (N7) to light-olive gray (5Y6/1) and microcrystalline. Amygdales toward the top of lava flows are abundant (as in Sample 554A-7-1, 63-66 cm), spheroidal to ellipsoidal, up to $8 \mathrm{~mm}$ across, and filled with goethite and smectite, or sparse (as in Sample 554A-8-1, 99-102 cm) in massive, light-olive gray (5Y 6/1) microcrystalline basalt. The base of a flow unit (Sample 554A-7-4, 25-28 cm) contains scattered spheroidal (1-2 mm diameter) vesicles. The samples from near the flow tops are sparsely plagioclase, or plagioclase-clinopyroxene-microphyric with microphenocrysts from $0.3 \times 0.02 \mathrm{~mm}$ to $0.6 \times 0.05 \mathrm{~mm}$ (Sample 554A-8-1, 99-102 cm). The groundmass is a finely microcrystalline felt of plagioclase needles averaging about $0.1 \times 0.03 \mathrm{~mm}$, with intergranular clinopyroxene $(0.01 \mathrm{~mm})$ and minor magnetite. A trace of an olivine pseudomorph occurs in Sample 554A-8-1, 99$102 \mathrm{~cm}$. From the base of a flow unit (Sample 554A-7-4, $25-28 \mathrm{~cm}$ ) the basalt is aphyric with altered glassy mesostasis. Felted plagioclase needles average $0.05 \times 0.01 \mathrm{~mm}$ with conspicuous intergranular clinopyroxene $(0.01 \mathrm{~mm})$.

Sample 554A-14-1, 36-39 cm is homogeneously microcrystalline basalt, medium light gray (N6), with rare vesicles.

\section{Mineral Composition}

The two samples from near flow tops are microphyric with labradorite and clinopyroxene microphenocrysts $(0.3$ $\times 0.2 \mathrm{~mm}$ ) in Sample 554A-7-1, 63-66 cm; sparse labradorite $\left(\mathrm{An}_{68}\right)$ laths attain $0.6 \times 0.05 \mathrm{~mm}$ in Sample $554 \mathrm{~A}-8-1,99-102 \mathrm{~cm}$. The groundmass is a finely microcrystalline (to $0.1 \times 0.03 \mathrm{~mm}$ ) felt of labradorite $\left(\mathrm{An}_{67}\right)$ needles with conspicuous intergranular subcalcic ferroaugite $(0.01 \mathrm{~mm})$ (Table 2$)$ and minor opaque iron-titanium oxide grains. Modal analysis indicates about $35 \%$ volume plagioclase, $55 \%$ clinopyroxene, and $10 \%$ opaque oxides and secondary minerals. Deep red smectite (after olivine) occurs in the second sample, which also contains some carbonate.

The sample from the middle of a flow (Sample 554A$7-3,10-12 \mathrm{~cm}$ ) is closely similar to the flow-top samples both in texture and modal mineralogy (Table 3). Electron microprobe analyses show that groundmass and phenocrystic plagioclase are closely similar in composition $\left(\mathrm{An}_{67}\right.$ and $\mathrm{An}_{68}$, respectively) and that pyroxene microphenocrysts are subcalcic ferroaugites $\left(\mathrm{Mg}_{33} \mathrm{Fe}_{46} \mathrm{Ca}_{21}\right)$.

Sample 554A-7-4, 25-28 cm (from the base of a flow) is aphyric with altered glassy patches with carbonate; the felted plagioclase needles average $0.05 \times 0.01 \mathrm{~mm}$, and there is conspicuous intergranular $(0.01 \mathrm{~mm})$ clinopyroxene.

Yellowish-gray (5Y 7/2) basaltic conglomerate in Sample $554 \mathrm{~A}-9-1,75-78 \mathrm{~cm}$, is plagioclase-phyric with laths up to $1.2 \times 0.9 \mathrm{~mm}$ in a groundmass of plagioclase needles $(0.06 \times 0.004 \mathrm{~mm})$ and interstitial clinopyroxene; the mode is obscured by much altered glassy mesostasis but the proportion of clinopyroxene to plagioclase appears to be less than in the flow units.

A sample from near the bottom of the hole (Sample 554A-14-1, 36-39 cm) contains rare vesicles, is patchily microphyric with nests of slender plagioclase laths and clinopyroxene crystals (up to $0.2 \mathrm{~mm}$ ) set in a microcrystalline ground of plagioclase needles $(0.03 \times 0.002 \mathrm{~mm})$, minutely interstitial clinopyroxene $(0.03 \mathrm{~mm})$, and glassy mesostasis (Plate 2, Fig. 1).

\section{Hole 555}

Basalt flows, hyaloclastites, and tuffs, occur in the lowest Unit (IV); interbeds of detrital sediment occur in 
Table 2. Summary of electron microprobe, optical, and X-ray diffraction analyses of basalts and associated rocks, Holes 554A and 555.

\begin{tabular}{|c|c|c|c|c|c|c|}
\hline \multirow[b]{3}{*}{$\begin{array}{c}\text { Sample } \\
\text { (interval in } \mathrm{cm} \text { ) }\end{array}$} & \multicolumn{5}{|c|}{$\begin{array}{c}\text { Primary minerals }{ }^{\mathrm{a}} \\
\text { Optical and electron microprobe analyses }\end{array}$} & \multirow{3}{*}{$\begin{array}{l}\text { Secondary minerals }{ }^{\mathrm{b}} \\
\text { X-ray diffraction analyses }\end{array}$} \\
\hline & \multicolumn{2}{|c|}{ Plagioclase (\%An) } & \multirow[b]{2}{*}{$\begin{array}{l}\text { Pyroxene } \\
\mathrm{Mg}: \mathrm{Fe}: \mathrm{Ca}\end{array}$} & \multirow[b]{2}{*}{$\begin{array}{c}\text { Olivine } \\
\text { (alteration) }\end{array}$} & \multirow[b]{2}{*}{ Opaques } & \\
\hline & $\begin{array}{l}\text { Ground- } \\
\text { mass }\end{array}$ & $\begin{array}{l}\text { Microphe- } \\
\text { nocrysts }\end{array}$ & & & & \\
\hline \multicolumn{7}{|l|}{ Hole 554A } \\
\hline $7-1,63-66$ & & & & & & Cel., cel./sap. \\
\hline $7-3,10-12$ & 67 & 68 & $\begin{array}{l}\text { S.c.f. aug. } \\
33: 46: 21\end{array}$ & & & Cel., cel./sap. \\
\hline $7-4,25-28$ & & & & & & Cel., cel./sap., cal. \\
\hline $8-1,99-102$ & & (80) & & Sap.-ps. & & Cel., cel./sap., cal., hem. \\
\hline $9-1,75-78$ & & & & Sap.-ps. & & Cel., cel./sap., cal., hem. \\
\hline $14-1,36-39$ & & & & & & Cel., cel./sap. \\
\hline \multirow{2}{*}{\multicolumn{7}{|c|}{$\begin{array}{l}\text { Hole } 555 \\
\text { Subunit IVa }\end{array}$}} \\
\hline & & & & & & \\
\hline $68-2,85-88$ & (55) & (56) & & Sap.-ps. & & Na-sap. > cel., chal. \\
\hline $68-3,122-126$ & (48) & (57) & & Sap.-ps. & & Ca-sap., cel., serp., cal. \\
\hline $69-2,37-40$ & (56) & 76 & E. di. $49: 10: 41$ & Sap.-ps. & & Ca-sap., cel., cal. \\
\hline $69-3,113-116$ & 48 & 74 & Aug. 46:15:39 & & Ti-mag. & Na-sap., cel., cal. \\
\hline $70-1,135-139$ & & & & & & Na-sap., cel. \\
\hline $75-2,91-94$ & & & & & & Anal. > Na-sap. > cel., hem. \\
\hline $76-2,67-70$ & & & & & & Anal. $>$ Na-sap. $>$ cel. $>$ hem. \\
\hline $76-2,117-120$ & & & & & & $\begin{array}{l}\text { Na-sap.> Ca-sap., anal., cel. } \\
\text { hem., ch., chal. }\end{array}$ \\
\hline $76-4,8-10$ & & & & & & $\begin{array}{l}\text { Na-sap., Ca-sap., cel./sap.. } \\
\text { cel., anal., hem., ch., chal. }\end{array}$ \\
\hline $76-5,22-24$ & & & & & & $\begin{array}{l}\text { anal. }>\text { Na-sap. > Ca-sap., cel., hem., } \\
\text { chal. }\end{array}$ \\
\hline $77-4,74-77$ & & & & Sap.-ps. & & (anal. > Na-sap., Ca-sap. $>$ cel., hem.) \\
\hline $77-5,144-147$ & (Tuffs) & & & & & Anal. > Na-sap. >cel., hem. \\
\hline $79-3,114-117$ & & & & & & Anal. $>$ Na-sap. $>$ cel., hem. \\
\hline $81-2,61-64$ & \multicolumn{2}{|c|}{ (Agglomerate) } & & & & $\begin{array}{l}\text { Na-sap.>Ca-sap., cel., anal., } \\
\text { ch., hem. }\end{array}$ \\
\hline $81-2,133-136$ & (56) & & & & & \\
\hline $81-3,110-113$ & 52 & 52 & Aug. 41:22:37 & & & Na-sap. > Ca-sap, anal., hem., ch. \\
\hline $82-1,36-38$ & & & & & & $\begin{array}{l}\text { Na-sap.> Ca-sap., cel., anal., } \\
\text { hem., ch. }\end{array}$ \\
\hline $82-1,136-139$ & (57) & & & & & \\
\hline $82-2,79-81$ & (Tuff) & & & & & $\begin{array}{l}\text { Na-sap.>cel., Ca-sap., anal., } \\
\text { hem., ch. }\end{array}$ \\
\hline $83-1,42-45$ & 45 & 45 & Aug. 46:22:32 & & Ti-mag. & \\
\hline \multicolumn{7}{|l|}{ Subunit IVb } \\
\hline $90-1,67-69$ & & & & & & Ca-sap. >cel. \\
\hline $90-1,125-128$ & (60) & & & & & \\
\hline $90-2,65-70$ & (57) & & & & & \\
\hline $91-1,94-96$ & & & & & & Ca-sap.>cel. \\
\hline $93-2,136-138$ & & & & & & Ca-sap.>cel., ch. \\
\hline \multicolumn{7}{|l|}{ Subunit IVc } \\
\hline $95-1,40-43$ & & & & & & Na-sap., Ca-sap. >cel., ch. \\
\hline $95-1,97-100$ & 74 & 74 & Aug. 44:28:28 & Fo82 & Ti-mag. & $\begin{array}{l}\text { Na-sap., Ca-sap. > cel., serp. ch. } \\
\text { (amph.) }\end{array}$ \\
\hline $96-2,95-98$ & (57) & (57) & & & & (sap., cel. serp., ch. amph.) \\
\hline $97-3,36-39$ & 66 & 66 & Aug. 47:14:39 & & Ti-mag. & (sap., cel., ch.) \\
\hline $97-6,80-83$ & (56) & (56) & & & & (sap., cel., ch.) \\
\hline $96-5,85-88$ & & & & & & (sap., cel., ch.) \\
\hline $96-5,108-110$ & 74 & 74 & Aug. 46:14:40 & Fo76 & Ilmenite & Ca-sap. > (cel., ch.) \\
\hline
\end{tabular}

Note: aug. = augite, s.c.f. aug. = subcalcic ferroaugite, e.di. = endiopside, $\mathrm{Mg}$-pig. = magnesium pigeonite, $\mathrm{Ti}$-mag. $=$ titaniferous magnetite, sap.-ps = saponite pseudomorphs after olivine, amph. = fibrous amphibole, chal. = chalcedony, cal. $=$ calcite, Nasap. $=$ sodium-saponite, Ca-sap. = calcium-saponite, sap. = saponite (optically), cel. = celadonite, cel./sap. = celadonite/saponite mixed layer mineral, nont. = nontronite, serp. $=$ serpentine, ch. $=$ chlorite, anal. $=$ analcime, hem. $=$ hematite.

a Optical determinations of anorthite content are shown in parentheses.

b Optical identifications of secondary minerals are shown in parentheses.

the two upper subunits (IVa, IVb) and a massive basalt dolerite forms the lowest (Unit IVc). We sectioned 33 samples of the basalt flows and 7 of volcaniclastic material from Subunits IVa and IVb, plus 12 samples of the massive basalt Subunit IVc. The 10 lava flow units of
Subunits IVa and IVb identified on shipboard are simple or composite, commonly with pillows, and mainly less than $1 \mathrm{~m}$ thick. The pillows show thin $(5-\mathrm{mm})$ rinds of altered dark glass which may be brecciated and vesicular interiors coarsening toward their centers. 
Table 3. Modal analyses of basalts from Holes 553A and 554A and of basalts and dolerites from Hole 555.

\begin{tabular}{|c|c|c|c|c|c|c|c|c|c|c|}
\hline \multirow{2}{*}{$\begin{array}{c}\text { Sample } \\
\text { (interval in cm) }\end{array}$} & \multirow[b]{2}{*}{ Brief lithology } & \multicolumn{3}{|c|}{ Phenocrysts (vol.\%) } & \multicolumn{5}{|c|}{ Groundmass (vol.\%) } & \multirow[b]{2}{*}{ Total } \\
\hline & & Plag. & Cpx. & Total & Plag. & Cpx. & Oliv. & Magnet. & Smectite & \\
\hline \multicolumn{11}{|l|}{ Hole 553A } \\
\hline $40-1,79-82$ & Sparsely phyric basalt & 1 & - & 1 & 35 & 51 & - & 7 & 6 & 99 \\
\hline $42-2,42-46$ & Sparsely phyric basalt & 2 & - & 2 & 41 & 41 & - & 4 & 12 & 98 \\
\hline $45-5,17-19$ & Sparsely phyric basalt & 1 & - & 1 & 36 & 51 & - & 8 & 4 & 99 \\
\hline $46-4,53-56$ & Aphyric basalt & - & - & - & 32 & 49 & - & 10 & 9 & 100 \\
\hline $48-3,97-101$ & $\begin{array}{l}\text { Moderately phyric } \\
\text { basalt }\end{array}$ & 3 & 1 & 4 & 29 & 51 & - & 8 & 8 & 96 \\
\hline $48-6,122-125$ & $\begin{array}{l}\text { Moderately phyric } \\
\text { basalt }\end{array}$ & 5 & - & 5 & 33 & 50 & tr & 5 & 7 & 95 \\
\hline $51-2,77-80$ & Aphyric basalt & - & - & - & 33 & 50 & - & 7 & 10 & 100 \\
\hline $55-3,113-116$ & Sparsely phyric basalt & 1 & - & 1 & 32 & 43 & - & 16 & 8 & 99 \\
\hline $58-1,89-91$ & Sparsely phyric basalt & tr & - & $\operatorname{tr}$ & 37 & 37 & - & 4 & 22 & 100 \\
\hline \multicolumn{11}{|l|}{ Hole 554A } \\
\hline $7-3,10-12$ & Sparsely phyric basalt & 1 & - & 1 & 29 & 53 & - & 13 & 4 & 99 \\
\hline \multicolumn{11}{|l|}{ Hole 555} \\
\hline $68-3,122-126$ & $\begin{array}{l}\text { Moderately phyric } \\
\text { basalt }\end{array}$ & 8 & 2 & 10 & 37 & 36 & $\operatorname{tr}$ & 7 & 10 & 90 \\
\hline $69-2,21-24$ & Glomerophyric basalt & 7 & 1 & 8 & 30 & 45 & $\operatorname{tr}$ & 7 & 10 & 92 \\
\hline $69-4,85-88$ & $\begin{array}{l}\text { Moderately phyric } \\
\text { basalt }\end{array}$ & 7 & 2 & 9 & 29 & 46 & tr & 7 & 9 & 91 \\
\hline $76-1,69-74$ & Dolerite & - & - & 0 & 38 & 40 & 0 & 1 & 21 & 100 \\
\hline $90-1,100-104$ & Aphyric basalt & - & - & 0 & 43 & 33 & tr & 4 & 20 & 100 \\
\hline $98-5,56-59$ & Olivine-dolerite & - & - & 0 & 49 & 37 & 6 & 3 & 5 & 100 \\
\hline $96-3,110-113$ & Olivine-dolerite & - & - & 0 & 46 & 40 & 5 & 3 & 6 & 100 \\
\hline
\end{tabular}

Note: $\mathrm{tr}=$ trace, dash indicates absence.

\section{General Features of Basalts of Subunits IVa and IVb}

Basalts above the pyroclastic sequence of Subunit IV show hues of greenish gray to greenish black (5G 2/1), with sporadic vesicles filled with smectite, chalcedony, calcite, or celadonite, and ranging from spheroidal to ellipsoidal, as at the base of a flow in Sample 555-70-1, $135-138 \mathrm{~cm}$. Cumulophyric plagioclase laths (up to 2.1 $\times 0.6 \mathrm{~mm}$ ) and clinopyroxene plates up to $6 \mathrm{~mm}$ across occur in the uppermost flow unit (Plate 2, Fig. 2), set in a microcrystalline groundmass of plagioclase needles averaging about $0.1 \times 0.02 \mathrm{~mm}$, unaltered intergranular clinopyroxene, and minor opaque ore. A trace of pseudomorphed olivine $(0.8 \times 0.6 \mathrm{~mm})$ occurs in Sample $555-69-3,113-116 \mathrm{~mm}$. Mesostasis is altered mainly to smectite and celadonite. Aphyric or sparsely microphyric basalts occur in the composite pillow lava units, and except for a fine plagioclase needle mesh $(0.1 \times 0.02 \mathrm{~mm})$ are heavily altered to smectite; glassy rinds are devitrified to smectite. Unaltered clinopyroxene granules occur, for example, in Samples 555-76-1, 10-13 cm and 555-76-5, 22-24 cm (Plate 2, Fig. 3). Beneath the volcaniclastic sequences of Subunit IVa, samples of composite pillow lavas away from glassy selvedges coarsen to subophitic dolerite (e.g., in the middle of a flow in Samples $555-81-3,110-113 \mathrm{~cm}$ and $555-82-1,136-139 \mathrm{~cm}$ ), in which plagioclase laths average $0.6 \times 0.07 \mathrm{~mm}$ and partly intergrown clinopyroxene averages $0.3 \mathrm{~mm}$. There is a little accessory magnetite, sporadic analcime, and much secondary clay.

Samples of the three units in Subunit IVb are mainly aphyric except for a plagioclase-microphyric $(1.5 \times$
$0.6 \mathrm{~mm}$ ) basalt in the middle of a flow in Sample $555-93-2,136-138 \mathrm{~cm}$. These basalts are hues of greenish gray becoming medium dark gray (N4) toward the lower part of the subunit. Vesicles rarely exceed $3 \mathrm{~mm}$ across and are mainly sparse even toward flow tops. Coarser (ophitic) textures with plagioclase laths average $1.0 \times 0.1 \mathrm{~mm}$ and intergrown clinopyroxene occur within the flows as in Sample 555-83-1, 42-45 cm (Plate 2, Fig. 5), but elsewhere the basalts are microcrystalline and equigranular with plagioclase needles averaging 0.04 $\times 0.004 \mathrm{~mm}$ (as in Sample 555-90-1, 67-69 cm), and intergranular clinopyroxene $(0.03 \times 0.06 \mathrm{~mm})$ in a smectite base.

\section{Pyroclastic Rocks of Subunits IVa and IVb}

We report on six samples of pyroclastic rocks including hyaloclastites or vitric tuffs from Sections 555-77-4, 77-5, 78-2, 79-3, 80-2, an agglomerate in Sample 555A81-2, 61-64 cm, and from Subunit IVb, a tuff in Sample $555-82-2,79-81 \mathrm{~cm}$. The hyaloclastic rocks consist of highly angular argillized glass particles up to $4 \mathrm{~mm}$ across varying from deep olive to grass green with yellow selvedges, clear, or containing scattered vesicles and crystallites of plagioclase. The glass is otherwise uniformly isotropic (with $n=1.566$ to $1.578 \pm 0.003$ ) to weakly anisotropic and shows networks of shrinkage cracks (Plate 2, Fig. 4); glass particles are generally separated by matrix rather than being self-supporting. Fragments of microphyric to aphyric lava occur between the glass particles and contain slender labradorite laths averaging $0.3 \times 0.03 \mathrm{~mm}$ and anhedra of clinopyroxene. These 
laths may be pseudomorphed by microcrystalline clay material (as in Sample 555-78-2, 97-100 cm).

An agglomerate in Sample 555-81-2, 61-64 cm contains angular fragments of dark brown cumulomicrophyric devitrified glassy lava with fluxioned margins and aligned microliths and vesicles. Clusters of labradorite laths and pseudomorphed(?) olivine grains reach $3 \mathrm{~mm}$ across. The matrix is a finely clastic aggregate of glass particles, feldspar crystals, and argillized material.

Tuff in Sample 555-82-2, 79-81 cm includes a particle of pillow lava showing a glassy rind and microphyric interior with plagioclase laths attaining $0.6 \times 0.03 \mathrm{~mm}$. The matrix consists of feldspar crystals, clinopyroxene, particles of altered glass, lava, quartz, apatite, biotite, and opaque oxides in a smectite-rich base. The feldspars include sodic plagioclase, microcline, and orthoclase and indicate an input from a wide variety of source rock other than basalt.

$\mathrm{X}$-ray diffraction (XRD) analyses (Table 2) show that the glassy fragmental rocks are altered to Na-saponite with celadonite, analcime, and hematite. The agglomerate and tuff are altered similarly although they also contain chlorite.

\section{Mineralogy of the Basalts of Subunits IVa and IVb}

Optical, electron microprobe, and XRD data are summarized in Table 2 and modal analyses of selected samples appear in Table 3. Plagioclase phenocrysts in the microphyric basalts of Subunit IVa range from $\mathrm{An}_{45}$ (andesine) to $\mathrm{An}_{76}$ (bytownite), and the groundmass plagioclase from $\mathrm{An}_{45}$ to $\mathrm{An}_{57}$ (labradorite). Coexisting plagioclases in the lower part of Subunit IVa show no compositional difference between groundmass and microphenocrysts (Table 4). Marked differences, however, occur in the upper part of the subunit and in the absence of zoning and suggest that the microphenocrysts are fractionated xenocrysts. In the same subunit an au- gitic pyroxene differs little from the lowest to the highest basalts and shows a range of $\mathrm{Mg}(41-46): \mathrm{Fe}(15-22)$ : $\mathrm{Ca}(32-39)$, except for Fe-poor endiopside (Mg 49 : Fe 10 : $\mathrm{Ca} 41$ ) in Sample $555-69-2,37-40 \mathrm{~cm}$. The opaque ore is titanomagnetite. Modal analyses of phyric basalts give $8-10 \%$ volume phenocrysts (plagioclase $7-8 \%$, clinopyroxene $1-2 \%$ ). Groundmass consists of $29-37 \%$ plagioclase, 36-46\% clinopyroxene, a trace of olivine, $7 \%$ titanomagnetite, and $9-10 \%$ secondary minerals.

\section{Subunit IVc}

Sample 555-95-1, 40-43 cm, near the chilled top of this single massive unit, is a greenish gray, aphyric microcrystalline basalt formed of a random mesh of plagioclase needles $(0.12 \times 0.02 \mathrm{~mm})(\sim 55 \%)$ and intergranular clinopyroxene $(\sim 30 \%)$ with minor opaque oxide, sparse orthopyroxene, and interstitial material replaced by smectite. In contrast to all other basic rocks of this and the other two sites (Sites 553 and 554), the underlying rocks are olivine-bearing ophitic to subophitic dolerites becoming coarse grained in the central position (Plate 2, Fig. 6). They are also conspicuous by change of color to bluish hues (mainly light bluish gray 5 to 7/1) compared with the greenish gray of most of the basalts. Vesicles are rare and reach $2 \mathrm{~mm}$ across. These dolerites consist of an equigranular intergrowth of labradorite (averaging $0.7 \times 0.07 \mathrm{~mm})$ and clinopyroxene $(0.3 \mathrm{~mm})$, with fresh and altered olivine crystals up to $0.6 \mathrm{~mm}$ across, minor opaque oxides, and an altered smectitic mesostasis. Plagioclase compositions are in the range labradorite to bytownite $\left(\mathrm{An}_{56}-\mathrm{An}_{74}\right)$, and the clinopyroxene is augite in the range $\mathrm{Mg} \mathrm{44-47:Fe} \mathrm{14-28:} \mathrm{Ca}$ 28-40. Microprobe analyses (Table 2) also show the olivine to be forsteritic $\left(\mathrm{Fo}_{76-82}\right)$, and the opaque ore is titanomagnetite. Modal analyses (Table 3 ) give plagioclase 46-49\% volume; augite, 37-40\%; titanomagnetite, $3 \%$; smectite and other secondaries, 5-6\%.

Table 4. Representative plagioclase analyses (Holes 552, 554A, and 555) normalized to eight oxygens.

\begin{tabular}{|c|c|c|c|c|c|c|c|c|}
\hline & \multicolumn{8}{|c|}{ Sample (interval in $\mathrm{cm})^{\mathrm{a}}$} \\
\hline & $\begin{array}{c}552-22-1, \\
137-141 \text { (MP) }\end{array}$ & $\begin{array}{c}552-22-1 \\
137-141(\mathrm{G})\end{array}$ & $\begin{array}{l}554 \mathrm{~A}-7-3 \\
10-12(\mathrm{G})\end{array}$ & $\begin{array}{c}554 \mathrm{~A}-7-3 \\
10-12 \text { (MP) }\end{array}$ & $\begin{array}{c}555-69-3, \\
113-116(\mathrm{G})\end{array}$ & $\begin{array}{c}\text { 555-69-3, } \\
113-116(\mathrm{P})\end{array}$ & $\begin{array}{c}555-83-1 \\
42-45 \text { (MP) }\end{array}$ & $\begin{array}{c}555-96-5 \\
108-110(\mathrm{O})\end{array}$ \\
\hline $\mathrm{SiO}_{2}$ & 52.75 & 59.29 & 51.52 & 52.37 & 56.53 & 49.71 & 57.57 & 49.54 \\
\hline $\mathrm{Al}_{2} \mathrm{O}_{3}$ & 28.87 & 24.46 & 29.13 & 29.68 & 25.97 & 30.97 & 26.24 & 30.75 \\
\hline $\mathrm{FeO}^{*}$ & 0.89 & 1.22 & 1.06 & 0.87 & 0.81 & 0.57 & 0.94 & 0.69 \\
\hline $\mathrm{CaO}$ & 12.42 & 8.09 & 13.45 & 13.80 & 9.43 & 15.08 & 9.30 & 15.01 \\
\hline $\mathrm{Na}_{2} \mathrm{O}$ & 4.08 & 6.44 & 3.74 & 3.64 & 5.76 & 2.89 & 6.26 & 2.87 \\
\hline $\mathrm{K}_{2} \mathrm{O}$ & 0.05 & 0.19 & 0.15 & 0.09 & 0.16 & 0.00 & 0.07 & 0.01 \\
\hline Total & 99.06 & 99.69 & 99.05 & 100.45 & 98.66 & 99.22 & 100.38 & 98.87 \\
\hline $\mathrm{Si}$ & 2.42 & 2.67 & 2.38 & 2.38 & 2.58 & 2.29 & 2.58 & 2.29 \\
\hline $\mathrm{Al}$ & 1.56 & 1.30 & 1.58 & 1.59 & 1.40 & 1.68 & 1.39 & 1.68 \\
\hline $\mathrm{Fe}$ & 0.03 & 0.05 & 0.04 & 0.03 & 0.03 & 0.02 & 0.04 & 0.03 \\
\hline $\mathrm{Ca}$ & 0.61 & 0.39 & 0.66 & 0.67 & 0.46 & 0.74 & 0.45 & 0.74 \\
\hline $\mathrm{Na}$ & 0.36 & 0.56 & 0.33 & 0.32 & 0.51 & 0.26 & 0.54 & 0.26 \\
\hline K & 0.01 & 0.01 & 0.01 & 0.01 & 0.01 & 0.00 & 0.01 & 0.00 \\
\hline An & 62.56 & 40.50 & 65.94 & 67.37 & 49.09 & 74.25 & 44.92 & 74.33 \\
\hline $\mathrm{Ab}$ & 37.13 & 58.36 & 33.17 & 32.13 & 51.99 & 25.75 & 54.67 & 25.67 \\
\hline Or & 0.31 & 1.14 & 0.89 & 0.50 & 0.92 & - & 0.40 & - \\
\hline
\end{tabular}

Note: Dash indicates absence. $\mathrm{FeO}^{*}$ includes $\mathrm{Fe}_{2} \mathrm{O}_{3}$ recalculated as $\mathrm{FeO}$.

${ }^{a}$ Grain type is indicated in parentheses after sample number. $G=$ groundmass, $M P=$ microphenocrysts, $P=$ phenocrysts, and $\mathrm{O}=$ ophitic. 


\section{Basaltic Rocks from Sites 552-555}

The samples of lava flows from all four sites are basalts showing similar ranges of textures and broadly similar primary mineralogy within the limits of sampling (see Tables 4-9). That is, most phenocrysts in phyric basalts are plagioclase mainly in the labradorite to bytownite range (with some andesine at Site 555), but a few are clinopyroxenes in the augite range. The groundmass of these and the aphyric basalts is an intergrowth of plagioclase, in the range andesine to labradorite, and mainly augite, although $\mathrm{Mg}$-pigeonite was also found in Hole 553A, and subcalcic ferroaugite in Hole 554A. The opaque ore is titaniferous magnetite. The primary mineralogy is generally similar in the lithologically distinct basal olivine-dolerite (IVc) of Hole 555, with essential labradorite, augite, and minor Ti-magnetite. However, here olivine is the characteristic accessory, with minor ilmenite. But traces of olivine also occur sporadically in the lava flows of Holes 553A, 554A, and 555. Modal analyses give similar ranges of primary minerals in the basalts of the four holes but the olivine-dolerites of

Table 5. Representative clinopyroxene analyses (Holes 552, 554A, and 555) normalized to six oxygens.

\begin{tabular}{|c|c|c|c|c|c|c|c|}
\hline & \multicolumn{7}{|c|}{ Sample (interval in $\mathrm{cm})^{\mathrm{a}}$} \\
\hline & $\begin{array}{c}552-22-1, \\
137-141(G)\end{array}$ & $\begin{array}{l}554 \mathrm{~A}-7-3 \\
10-12(\mathrm{G})\end{array}$ & $\begin{array}{l}555-69-2 \\
37-40(0)\end{array}$ & $\begin{array}{c}555-69-3 \\
113-116(\mathrm{G})\end{array}$ & $\begin{array}{c}555-81-3 \\
110-113(G)\end{array}$ & $\begin{array}{c}555-95-1 \\
97-100(\mathrm{O})\end{array}$ & $\begin{array}{c}555-96-5 \\
108-110(0)\end{array}$ \\
\hline $\mathrm{SiO}_{2}$ & 49.02 & 49.16 & 53.15 & 51.18 & 50.50 & 51.87 & 52.72 \\
\hline $\mathrm{Al}_{2} \mathrm{O}_{3}$ & 3.69 & 1.11 & 2.07 & 2.09 & 2.23 & 1.22 & 1.89 \\
\hline $\mathrm{FeO} *^{*}$ & 12.58 & 26.91 & 6.15 & 9.30 & 13.21 & 16.99 & 8.77 \\
\hline $\mathrm{MnO}$ & 0.04 & 0.61 & 0.05 & 0.20 & 0.33 & 0.26 & 0.20 \\
\hline $\mathrm{MgO}$ & 13.27 & 10.67 & 16.99 & 15.71 & 14.15 & 15.00 & 16.09 \\
\hline $\mathrm{CaO}$ & 19.21 & 9.67 & 19.92 & 18.17 & 17.49 & 13.65 & 19.87 \\
\hline $\mathrm{TiO}_{2}$ & 1.92 & 0.78 & 0.34 & 0.83 & 0.82 & 0.43 & 0.60 \\
\hline $\mathrm{Cr}_{2} \mathrm{O}_{3}$ & 0.20 & 0.00 & 0.69 & 0.22 & 0.18 & 0.00 & 0.07 \\
\hline Total & 99.93 & 98.91 & 99.36 & 97.70 & 98.91 & 99.42 & 100.21 \\
\hline $\mathrm{Si}_{\text {IV }}$ & 1.85 & 1.96 & 1.96 & 1.94 & 1.92 & 1.97 & 1.95 \\
\hline $\mathrm{Al}_{\mathrm{VI}}^{\mathrm{IV}}$ & 0.15 & 0.04 & 0.04 & 0.06 & 0.08 & 0.03 & 0.05 \\
\hline $\mathrm{Al}^{\mathrm{VI}}$ & 0.02 & 0.01 & 0.05 & 0.03 & 0.02 & 0.03 & 0.03 \\
\hline $\mathrm{Fe}$ & 0.40 & 0.90 & 0.19 & 0.30 & 0.42 & 0.54 & 0.27 \\
\hline Mn & 0.00 & 0.02 & 0.00 & 0.01 & 0.01 & 0.01 & 0.01 \\
\hline $\mathrm{Mg}$ & 0.75 & 0.63 & 0.93 & 0.89 & 0.80 & 0.85 & 0.89 \\
\hline $\mathrm{Ca}$ & 0.78 & 0.41 & 0.79 & 0.74 & 0.71 & 0.56 & 0.79 \\
\hline $\mathrm{Ti}$ & 0.06 & 0.02 & 0.01 & 0.02 & 0.02 & 0.01 & 0.02 \\
\hline $\mathrm{Cr}$ & 0.01 & 0.00 & 0.02 & 0.01 & 0.01 & 0.00 & 0.00 \\
\hline $\mathrm{Mg} / \mathrm{Mg}+\mathrm{Fe}^{2+} \%$ & 65.22 & 41.40 & 83.13 & 75.04 & 65.60 & 61.12 & 76.56 \\
\hline Wo & 40.47 & 21.23 & 41.21 & 38.41 & 36.84 & 28.59 & 40.47 \\
\hline En & 38.86 & 32.61 & 48.87 & 46.22 & 41.43 & 43.65 & 45.57 \\
\hline Fs & 20.68 & 46.16 & 9.92 & 15.37 & 21.72 & 27.76 & 13.95 \\
\hline
\end{tabular}

Note: $\mathrm{FeO}^{*}$ includes $\mathrm{Fe}_{2} \mathrm{O}_{3}$ recalculated as $\mathrm{FeO}$.

${ }^{\mathrm{a}}$ Grain type is indicated in parentheses after sample number. $\mathrm{G}=$ groundmass, $\mathrm{O}=$ ophitic.

Table 6. Representative plagioclase analyses (Hole 553A) normalized to eight oxygens.

\begin{tabular}{|c|c|c|c|c|c|c|c|c|c|}
\hline & \multicolumn{9}{|c|}{ Sample (interval in cm) ${ }^{\mathbf{a}}$} \\
\hline & $\begin{array}{c}553 \mathrm{~A}-39-1 \\
2-4(\mathrm{G})\end{array}$ & $\begin{array}{l}553 \mathrm{~A}-39-1, \\
2-4 \text { (MP) }\end{array}$ & $\begin{array}{l}553 A-43-1, \\
20-23(G)\end{array}$ & $\begin{array}{l}553 \mathrm{~A}-43-1 \\
20-23 \text { (MP) }\end{array}$ & $\begin{array}{c}553 \mathrm{~A}-46-1, \\
121-125(\mathrm{G})\end{array}$ & $\begin{array}{l}\text { 553A-51-1, } \\
54-57(\mathrm{G})\end{array}$ & $\begin{array}{l}\text { 553A-51-1, } \\
54-57 \text { (MP) }\end{array}$ & $\begin{array}{l}553 \mathrm{~A}-57-2, \\
35-37(\mathrm{G})\end{array}$ & $\begin{array}{l}553 \mathrm{~A}-57-2, \\
35-37 \text { (MP) }\end{array}$ \\
\hline $\mathrm{SiO}_{2}$ & 52.26 & 47.06 & 51.57 & 48.99 & 52.56 & 49.81 & 47.41 & 49.07 & 45.95 \\
\hline $\mathrm{Al}_{2} \mathrm{O}_{3}$ & 27.71 & 31.27 & 29.33 & 30.07 & 29.51 & 27.71 & 32.68 & 29.65 & 31.92 \\
\hline $\mathrm{FeO}{ }^{*}$ & .2 .17 & 0.70 & 0.95 & 0.69 & 1.13 & 1.37 & 0.62 & 1.02 & 0.50 \\
\hline $\mathrm{CaO}$ & 12.16 & 16.45 & 14.00 & 14.94 & 13.40 & 13.15 & 16.94 & 12.28 & 16.37 \\
\hline $\mathrm{Na}_{2} \mathrm{O}$ & 4.05 & 1.99 & 3.34 & 2.57 & 4.20 & 3.41 & 1.70 & 3.40 & 1.49 \\
\hline $\mathrm{K}_{2} \mathrm{O}$ & 0.06 & 0.03 & 0.00 & 0.01 & 0.08 & 0.07 & 0.00 & 0.05 & 0.03 \\
\hline Total & 98.41 & 97.50 & 99.19 & 97.27 & 100.88 & 95.52 & 99.35 & 92.77 & 96.26 \\
\hline $\mathrm{Si}$ & 2.43 & 2.22 & 2.37 & 2.30 & 2.38 & 2.38 & 2.19 & 2.41 & 2.19 \\
\hline Al & 1.52 & 1.74 & 1.59 & 1.67 & 1.57 & 1.56 & 1.78 & 1.56 & 1.79 \\
\hline $\mathrm{Fe}$ & 0.08 & 0.03 & 0.04 & 0.03 & 0.04 & 0.06 & 0.02 & 0.04 & 0.02 \\
\hline $\mathrm{Ca}$ & 0.61 & 0.83 & 0.69 & 0.75 & 0.65 & 0.67 & 0.84 & 0.65 & 0.84 \\
\hline $\mathrm{Na}$ & 0.37 & 0.18 & 0.30 & 0.23 & 0.37 & 0.32 & 0.15 & 0.32 & 0.14 \\
\hline K & 0.01 & 0.00 & 0.00 & 0.00 & 0.01 & 0.01 & 0.00 & 0.01 & 0.01 \\
\hline An & 62.11 & 82.05 & 69.84 & 76.27 & 63.48 & 67.81 & 84.68 & 66.43 & 85.66 \\
\hline $\mathrm{Ab}$ & 37.47 & 17.95 & 30.16 & 23.73 & 36.04 & 31.79 & 15.32 & 33.26 & 14.14 \\
\hline Or & 0.41 & - & - & - & 0.49 & 0.40 & - & 0.31 & 0.20 \\
\hline
\end{tabular}

Note: Dash indicates absence. $\mathrm{FeO} *$ includes $\mathrm{Fe}_{2} \mathrm{O}_{3}$ recalculated as $\mathrm{FeO}$.

${ }^{\mathrm{a}}$ Grain type indicated in parentheses after sample number. $\mathrm{G}=$ groundmass, $\mathrm{MP}=$ microphenocrysts. 
Table 7. Representative clinopyroxene analyses (Hole 553A) normalized to six oxygens.

\begin{tabular}{|c|c|c|c|c|c|c|}
\hline & \multicolumn{6}{|c|}{ Sample (interval in $\mathrm{cm})^{\mathbf{a}}$} \\
\hline & $\begin{array}{l}\text { 553A-45-1, } \\
44-47 \text { (MP) }\end{array}$ & $\begin{array}{l}553 \mathrm{~A}-46-1 \\
121-125(\mathrm{G})\end{array}$ & $\begin{array}{c}553 \mathrm{~A}-46-1, \\
121-125(\mathrm{G})\end{array}$ & $\begin{array}{l}553 \mathrm{~A}-51-1, \\
54-57(\mathrm{G})\end{array}$ & $\begin{array}{c}\text { 553A-55-3, } \\
113-116(\mathrm{G})\end{array}$ & $\begin{array}{l}553 \mathrm{~A}-57-2, \\
35-37(\mathrm{G})\end{array}$ \\
\hline $\mathrm{SiO}_{2}$ & 50.71 & 51.91 & 52.93 & 50.75 & 51.80 & 48.32 \\
\hline $\mathrm{Al}_{2} \mathrm{O}_{3}$ & 2.35 & 1.60 & 1.01 & 2.06 & 1.81 & 6.79 \\
\hline $\mathrm{FeO}^{*}$ & 10.13 & 15.53 & 20.97 & 12.35 & 10.26 & 9.27 \\
\hline $\mathrm{MnO}$ & 0.34 & 0.38 & 0.56 & 0.44 & 0.21 & 0.18 \\
\hline $\mathrm{MgO}$ & 15.58 & 14.63 & 18.33 & 15.49 & 16.41 & 13.49 \\
\hline $\mathrm{CaO}$ & 15.26 & 15.68 & 6.83 & 16.01 & 17.34 & 13.69 \\
\hline $\mathrm{TiO}_{2}$ & 0.47 & 0.54 & 0.47 & 0.44 & 0.30 & 0.47 \\
\hline $\mathrm{Cr}_{2} \mathrm{O}_{3}$ & - & 0.00 & 0.00 & 0.07 & 0.08 & - \\
\hline Total & 94.84 & 100.27 & 101.10 & 96.61 & 98.21 & 92.21 \\
\hline $\mathrm{Si}_{\mathrm{IV}}$ & 1.97 & 1.95 & 1.97 & 1.94 & 1.95 & 1.91 \\
\hline $\mathrm{Al}_{\mathrm{v}}^{\mathrm{IV}}$ & 0.03 & 0.05 & 0.03 & 0.06 & 0.05 & 0.09 \\
\hline $\mathrm{Al}^{\mathrm{VI}}$ & 0.08 & 0.02 & 0.01 & 0.03 & 0.03 & 0.23 \\
\hline $\mathrm{Fe}$ & 0.33 & 0.49 & 0.65 & 0.40 & 0.32 & 0.31 \\
\hline $\mathrm{Mn}$ & 0.01 & 0.01 & 0.02 & 0.01 & 0.01 & 0.01 \\
\hline $\mathrm{Mg}$ & 0.90 & 0.82 & 1.02 & 0.88 & 0.92 & 0.79 \\
\hline $\mathrm{Ca}$ & 0.63 & 0.63 & 0.27 & 0.66 & 0.70 & 0.58 \\
\hline $\mathrm{Ti}$ & 0.01 & 0.02 & 0.01 & 0.01 & 0.01 & 0.01 \\
\hline $\mathrm{Cr}$ & - & 0.00 & 0.00 & 0.00 & 0.00 & - \\
\hline $\mathrm{Mg} / \mathrm{Mg}+\mathrm{Fe}^{2+} \%$ & 73.25 & 62.67 & 60.92 & 69.09 & 74.06 & 72.18 \\
\hline Wo & 34.01 & 32.54 & 14.04 & 33.92 & 35.99 & 34.48 \\
\hline En & 48.34 & 42.28 & 52.37 & 45.66 & 47.40 & 47.29 \\
\hline Fs & 17.65 & 25.18 & 33.59 & 20.42 & 16.61 & 18.23 \\
\hline
\end{tabular}

Note: Dash indicates no data. $\mathrm{FeO}^{*}$ includes $\mathrm{Fe}_{2} \mathrm{O}_{3}$ recalculated as $\mathrm{FeO}$.

${ }^{a}$ Grain type indicated in parentheses after sample number. $\mathrm{G}=$ groundmass, $\mathrm{MP}=$ microphenocryst.

Table 8. Representative olivine analyses (Hole 555) normalized to four oxygens.

\begin{tabular}{lcr}
\hline & \multicolumn{2}{c}{ Sample } \\
& \multicolumn{2}{c}{ (interval in cm) } \\
\cline { 2 - 3 } & $555-95-1$, & $555-96-5$ \\
& $97-100$ & $108-110$ \\
\hline $\mathrm{SiO}_{2}$ & 39.84 & 38.60 \\
$\mathrm{FeO}^{*}$ & 16.79 & 22.26 \\
$\mathrm{MnO}$ & 0.31 & 0.21 \\
$\mathrm{MgO}$ & 42.58 & 38.59 \\
$\mathrm{CaO}$ & 0.41 & 0.33 \\
$\mathrm{TiO} \mathrm{O}_{2}$ & 0.00 & 0.01 \\
$\mathrm{Cr}_{2} \mathrm{O}_{3}$ & 0.00 & 0.07 \\
$\mathrm{Total}$ & 99.93 & 100.07 \\
& & \\
$\mathrm{Si}$ & 1.01 & 1.00 \\
$\mathrm{Fe}$ & 0.36 & 0.48 \\
$\mathrm{Mn}$ & 0.01 & 0.01 \\
$\mathrm{Mg}$ & 1.61 & 1.49 \\
$\mathrm{Ca}$ & 0.01 & 0.01 \\
$\mathrm{Ti}$ & - & 0.00 \\
$\mathrm{Cr}$ & - & 0.01 \\
$\mathrm{Mg} / \mathrm{Mg}+\mathrm{Fe}^{2+} \%$ & 81.87 & 75.53 \\
\hline
\end{tabular}

Note: Dash indicates no data. $\mathrm{FeO}^{*}$ includes $\mathrm{Fe}_{2} \mathrm{O}_{3}$ recalculated as $\mathrm{FeO}$.

Hole 555 contain higher plagioclase and lower clinopyroxene than the basalts. No systematic downhole variations in mineralogy were detected at any of the sites.

Electron microprobe analyses reveal consistent differences between groundmass and phenocrystic plagioclase in Hole 553A basalts and also in the one sample examined from Hole 552. These differences are also apparent in the upper part of Subunit IVa but are absent from the remainder of Hole 555 basalts and dolerites, and they do not appear in the one analyzed sample from Hole 554A. The strongly calcic nature of the phenocrysts in Hole 553A lavas and, in all cases, a lack of zonation coupled with, in some cases, marginal resorption, would seem to rule out an intratelluric origin, suggesting instead that the phenocrysts were fractionated from a more primitive magma. Thus these xenocrystic lavas of Hole 553A appear to share some specific petrographical characteristics with the poorly sampled lavas of Hole 552 and the uppermost lavas of Hole 555 but not with the other basalts examined.

\section{CHEMISTRY}

\section{Major Oxides}

We report 16 whole-rock analyses, made by beta-probe, of the least-altered basalt samples of Hole 553A. All have $\mathrm{Fe}_{2} \mathrm{O}_{3}$ less than $7.0 \%$ and $\left(\mathrm{H}_{2} \mathrm{O}^{+}+\mathrm{CO}_{2}\right)$ contents of less than $5 \%$ (except two samples which slightly exceeded that amount), which is an acceptable limit for altered rocks. Three similarly least-altered basalts were analyzed from Hole 554A and two from Hole 555. Six olivine-dolerites were analyzed from the basal Unit IVc of Hole 555. Two vitroclastic tuffs (from Holes 553A and 555) were also analyzed for comparative purposes; they contain high $\mathrm{Fe}_{2} \mathrm{O}_{3}$ and $\mathrm{H}_{2} \mathrm{O}$. Results are tabulated in Tables 10-13, and expressed on a moisture-free basis; $\mathrm{H}_{2} \mathrm{O}\left(-105^{\circ} \mathrm{C}\right)$ contents are listed separately.

There is little variation in major oxides in the basalts of Hole 553A. No significant variation is apparent with depth of sample or between flow tops, middles, or bases. The ranges noted in Table 10 reflect variations in secondary minerals (vugs, mesostasis, alteration); the intrasample variation in, for example, vesicle proportions 
Table 9. Representative analyses of opaque oxides from Holes 552, 553A, and 555.

\begin{tabular}{lrrrrrr}
\hline & \multicolumn{5}{c}{ Sample (interval in cm) } \\
\cline { 2 - 7 } & $552-22-1$, & $553 \mathrm{~A}-39-1$, & $553 \mathrm{~A}-55-3$, & $555-69-3$, & $555-95-1$, & $555-96-5$, \\
& $137-141$ & $2-4$ & $113-116$ & $113-116$ & $97-100$ & $108-110$ \\
\hline $\mathrm{SiO}_{2}$ & 0.41 & 0.65 & 0.35 & 0.60 & 1.40 & 0.55 \\
$\mathrm{TiO}_{2}$ & 21.91 & 17.39 & 13.20 & 26.33 & 23.13 & 50.11 \\
$\mathrm{Al}_{2} \mathrm{O}_{3}$ & 2.46 & 1.05 & 1.93 & 1.50 & 1.26 & 0.36 \\
$\mathrm{Cr}_{2} \mathrm{O}_{3}$ & 0.12 & 0.17 & 0.00 & 0.23 & 0.01 & 0.16 \\
$\mathrm{Fe}_{2} \mathrm{O}_{3}$ & 22.57 & 30.76 & 38.57 & 12.12 & 17.72 & - \\
$\mathrm{FeO}$ & 49.06 & 44.67 & 39.09 & 52.63 & 51.30 & 45.51 \\
$\mathrm{MnO}$ & 0.48 & 0.23 & 2.32 & 1.29 & 1.12 & 0.42 \\
$\mathrm{MgO}$ & 1.56 & 1.28 & 1.03 & 0.83 & 0.85 & 2.33 \\
$\mathrm{Total}$ & 98.57 & 96.20 & 96.49 & 95.53 & 96.79 & 99.44 \\
& & & & & & \\
$\mathrm{Si}$ & 0.12 & 0.20 & 0.11 & 0.19 & 0.43 & 0.03 \\
$\mathrm{Ti}$ & 4.91 & 4.04 & 3.06 & 6.11 & 5.30 & 1.90 \\
$\mathrm{Al}$ & 0.86 & 0.38 & 0.70 & 0.55 & 0.45 & 0.02 \\
$\mathrm{Cr}$ & 0.03 & 0.04 & 0.00 & 0.06 & 0.00 & 0.01 \\
$\mathrm{Fe}{ }^{3+}$ & 5.06 & 7.14 & 8.96 & 2.81 & 4.07 & - \\
$\mathrm{Fe}$ & 12.21 & 11.53 & 10.09 & 13.58 & 13.08 & 1.92 \\
$\mathrm{Mn}$ & 0.12 & 0.06 & 0.61 & 0.34 & 0.29 & 0.02 \\
$\mathrm{Mg}$ & 0.69 & 0.59 & 0.47 & 0.38 & 0.39 & 0.18 \\
$\mathrm{No}$ of & 32 & 32 & 32 & 32 & 32 & 6 \\
\hline
\end{tabular}

Note: Dash indicates no data. $\mathrm{Fe}^{3+}$ and $\mathrm{Fe}^{2+}$ estimated on the basis of 24 cations per unit cell.

Table 10. Whole-rock chemical analyses ${ }^{\mathrm{a}}$ of basalts and a tuff from Hole 553A (wt.\%). ${ }^{\mathrm{b}}$

\begin{tabular}{|c|c|c|c|c|c|c|c|c|c|c|c|c|c|c|c|c|}
\hline $\begin{array}{l}\text { Core-Section } \\
\text { (interval in } \mathrm{cm} \text { ) }\end{array}$ & Lithology & $\mathrm{SiO}_{2}$ & $\mathrm{Al}_{2} \mathrm{O}_{3}$ & $\mathrm{Fe}_{2} \mathrm{O}_{3}$ & $\mathrm{FeO}$ & $\mathrm{MgO}$ & $\mathrm{CaO}$ & $\mathrm{Na}_{2} \mathrm{O}$ & $\mathrm{K}_{2} \mathrm{O}$ & $\mathrm{H}_{2} \mathrm{O}^{+}$ & $\mathrm{CO}_{2}$ & $\mathrm{TiO}_{2}$ & $\mathrm{MnO}$ & $\mathrm{P}_{2} \mathrm{O}_{5}$ & Total & $\mathrm{H}_{2} \mathrm{O}^{-}$ \\
\hline $38-2,56-59$ & Basalt & 52.0 & 14.0 & 6.48 & 6.82 & 7.61 & 9.96 & 2.46 & 0.16 & 1.61 & 0.13 & 1.32 & 0.32 & 0.10 & 102.97 & 2.50 \\
\hline $40-1,79-82$ & Basalt & 54.7 & 14.0 & 6.65 & 6.21 & 5.24 & 8.57 & 2.88 & 0.10 & 1.31 & c & 1.46 & 0.38 & 0.14 & 101.64 & 1.55 \\
\hline $45-1,44-47$ & Tuff & 52.1 & 11.4 & 16.3 & 1.32 & 5.13 & 4.11 & 2.11 & 1.14 & 4.95 & c & 1.45 & 0.13 & 0.04 & 100.18 & 8.55 \\
\hline $45-5,17-19$ & $\begin{array}{l}\text { Basalt, } \\
\text { cpx-rich }\end{array}$ & 50.3 & 13.8 & 5.50 & 7.52 & 7.28 & 11.3 & 2.16 & 0.06 & 1.44 & 0.00 & 1.19 & 0.42 & 0.13 & 101.10 & 2.02 \\
\hline $46-1,121-125$ & Basalt & 50.3 & 13.5 & 3.47 & 9.54 & 7.35 & 11.5 & 2.01 & 0.05 & 0.87 & 0.06 & 1.13 & 0.47 & 0.17 & 100.42 & 0.79 \\
\hline $46-3,56-59$ & Basalt & 50.8 & 13.7 & 3.62 & 9.34 & 7.32 & 11.5 & 2.10 & 0.06 & 0.81 & $\mathrm{c}$ & 1.16 & 0.49 & 0.11 & 101.01 & 0.85 \\
\hline $46-4,53-56$ & Basalt & 50.2 & 13.5 & 4.08 & 8.61 & 7.38 & 11.5 & 2.03 & 0.06 & 0.90 & c & 1.13 & 0.37 & 0.12 & 99.88 & 1.10 \\
\hline $47-3,73-76$ & Basalt & 49.7 & 13.2 & 6.26 & 8.18 & 6.90 & 10.1 & 2.34 & 0.08 & 1.61 & 0.02 & 1.52 & 0.38 & 0.12 & 100.41 & 2.18 \\
\hline $48-2,60-65$ & Basalt & 49.6 & 13.4 & 5.10 & 9.03 & 6.86 & 10.4 & 2.20 & 0.06 & 1.38 & 0.00 & 1.50 & 0.53 & 0.12 & 100.18 & 1.65 \\
\hline $51-1,54-57$ & Basalt & 49.1 & 13.7 & 6.34 & 6.49 & 7.92 & 11.0 & 2.08 & 0.06 & 1.75 & 0.05 & 0.99 & 0.38 & 0.08 & 99.94 & 2.23 \\
\hline $51-2,77-80$ & Basalt & 50.2 & 13.8 & 4.3 & 7.89 & 7.85 & 11.2 & 2.06 & 0.06 & 1.45 & 0.06 & 1.01 & 0.38 & 0.07 & 100.33 & 1.66 \\
\hline $53-1,77-80$ & Basalt & 49.8 & 13.9 & 5.80 & 6.22 & 8.26 & 10.9 & 2.17 & 0.06 & 1.62 & 0.30 & 1.07 & 0.40 & 0.08 & 100.58 & 3.24 \\
\hline $54-4,81-85$ & Basalt & 50.0 & 13.2 & 4.78 & 9.64 & 6.56 & 10.2 & 2.33 & 0.06 & 1.19 & 0.14 & 1.35 & 0.54 & 0.12 & 100.11 & 1.94 \\
\hline $55-5,92-95$ & Basalt & 50.0 & 13.6 & 4.19 & 8.65 & 7.18 & 11.1 & 2.08 & 0.05 & 0.89 & 0.20 & 1.15 & 0.50 & 0.08 & 99.67 & 1.78 \\
\hline $55-6,44-47$ & Basalt & 50.4 & 13.7 & 4.04 & 8.87 & 7.02 & 11.1 & 2.16 & 0.06 & 1.08 & 0.16 & 1.17 & 0.50 & 0.09 & 100.35 & 1.32 \\
\hline $56-1,3-6$ & Basalt & 48.7 & 13.8 & 3.66 & 8.33 & 7.18 & 11.1 & 2.16 & 0.06 & 1.85 & 0.96 & 1.18 & 0.65 & 0.08 & 99.71 & 2.48 \\
\hline \multirow[t]{3}{*}{$59-2,115-118$} & Basalt & 49.5 & 15.6 & 5.06 & 5.12 & 8.12 & 10.7 & 2.16 & 0.04 & 2.26 & 0.00 & 1.08 & 0.33 & 0.07 & 100.04 & 2.95 \\
\hline & Mean ${ }^{d}$ & 50.3 & 13.8 & 4.96 & 7.90 & 7.25 & 10.7 & 2.21 & 0.07 & 1.37 & 0.16 & 1.28 & 0.44 & 0.11 & 100.50 & 1.89 \\
\hline & Range $^{\mathrm{d}}$ & 6.0 & 2.4 & 3.18 & 4.52 & 3.02 & 3.03 & 0.87 & 0.12 & 1.45 & 0.96 & 0.47 & 0.33 & 0.10 & 3.30 & 2.16 \\
\hline
\end{tabular}

Analysts: Dawn Hutchison, A. E. Davis and K. A. Holmes.

${ }^{b}$ Results expressed on a moisture-free basis.

c Not determined (insufficient sample).

d Excluding the tuff sample.

and infillings might otherwise suggest more variations in bulk chemistry. The greatest ranges in terms of the means of the six major oxides are shown by $\mathrm{Fe}_{2} \mathrm{O}_{3}, \mathrm{FeO}$, $\mathrm{MgO}$, and $\mathrm{CaO}$. The means of the basalts compare favorably with tholeiitic basalt (Nockolds' means of 137 analyses, 1954) and ocean-floor tholeiitic basalt (Nockolds et al., 1978) (Table 11).

The $\mathrm{K}_{2} \mathrm{O}$ of the Hole $553 \mathrm{~A}$ basalts is considerably lower than that of geographically close olivine-basalts of DSDP Leg 12, Sites 116 and 117 (Laughton et al., 1972), but compares closely with a basalt from the MidAtlantic Ridge, from DSDP Leg 49 (Luyendyk, Cann, et al., 1979). Many other basalts from the site, however, contain much higher $\mathrm{Fe}_{2} \mathrm{O}_{3}$ than do the present samples.

Analyses of three basalts from Hole 554A (Table 12) are closely similar except for higher $\mathrm{Fe}_{2} \mathrm{O}_{3}$ in Sample $554 \mathrm{~A}-7-1,63-66 \mathrm{~cm}$. Their means are close to the Hole $553 \mathrm{~A}$ basalts except for significantly higher $\mathrm{K}_{2} \mathrm{O}$ and lower $\mathrm{MgO}$ in the former.

Two Hole 555 basalts (Table 13) approach the means of the other two sites. $\mathrm{MgO}$ contents of Hole 555 basalts, however, are closer to those of Hole 554A; also their $\mathrm{Na}_{2} \mathrm{O}$ and $\mathrm{TiO}_{2}$ contents are a little higher than at the other sites. Five olivine-dolerites from Hole 555 con- 
Table 11. Comparison of major oxides of basalts from Holes 409 and 553A and ocean floor and tholeitic basalts.

\begin{tabular}{lllllllllllll}
\hline & $\mathrm{SiO}_{2}$ & $\mathrm{Al}_{2} \mathrm{O}_{3}$ & $\mathrm{Fe}_{2} \mathrm{O}_{3}$ & $\mathrm{FeO}$ & $\mathrm{MgO}$ & $\mathrm{CaO}$ & $\mathrm{Na}_{2} \mathrm{O}$ & $\mathrm{K}_{2} \mathrm{O}$ & $\mathrm{H}_{2} \mathrm{O}^{+}$ & $\mathrm{TiO}_{2}$ & $\mathrm{MnO}^{2}$ & $\mathrm{P}_{2} \mathrm{O}_{5}$ \\
\hline Hole 553A basalts & 50.3 & 13.8 & 4.96 & 7.90 & 7.25 & 10.7 & 2.21 & 0.07 & 1.37 & 1.28 & 0.44 & 0.11 \\
Tholeiitic basalts & 50.83 & 14.07 & 2.88 & 9.06 & 6.34 & 10.42 & 2.23 & 0.82 & 0.91 & 2.03 & 0.18 & 0.23 \\
$\begin{array}{l}\text { Ocean-floor tholeiitic } \\
\text { basalts }\end{array}$ & 49.99 & 15.65 & 1.74 & 8.06 & 7.98 & 11.36 & 2.70 & 0.19 & 0.60 & 1.40 & 0.19 & 0.23 \\
Hole 409 basalts & 49.91 & 14.09 & 2.46 & 8.14 & 8.15 & 11.89 & 2.21 & 0.07 & 0.69 & 1.17 & 0.17 & 0.14 \\
\hline
\end{tabular}

Table 12. Whole-rock chemical analyses ${ }^{\mathrm{a}}$ of basalts from Hole 554A (wt.\%) ${ }^{\mathrm{b}}$.

\begin{tabular}{|c|c|c|c|c|c|c|c|c|c|c|c|c|c|c|c|c|}
\hline $\begin{array}{l}\text { Core-Section } \\
\text { (interval in cm) }\end{array}$ & Lithology & $\mathrm{SiO}_{2}$ & $\mathrm{Al}_{2} \mathrm{O}_{3}$ & $\mathrm{Fe}_{2} \mathrm{O}_{3}$ & $\mathrm{FeO}$ & $\mathrm{MgO}$ & $\mathrm{CaO}$ & $\mathrm{Na}_{2} \mathrm{O}$ & $\mathrm{K}_{2} \mathrm{O}$ & $\mathrm{H}_{2} \mathrm{O}^{+}$ & $\mathrm{CO}_{2}$ & $\mathrm{TiO}_{2}$ & $\mathrm{MnO}$ & $\mathrm{P}_{2} \mathrm{O}_{5}$ & Total & $\mathrm{H}_{2} \mathrm{O}^{-}$ \\
\hline $7-1,63-66$ & Basalt & 50.8 & 13.6 & 6.34 & 6.87 & 6.58 & 11.4 & 2.14 & 0.34 & 0.74 & 0.06 & 1.08 & 0.30 & 0.08 & 100.33 & 0.74 \\
\hline $7-3,10-12$ & Basalt & 51.5 & 14.0 & 3.85 & 7.79 & 6.70 & 11.7 & 2.28 & 0.19 & 0.77 & 0.02 & 1.06 & 0.31 & 0.07 & 100.24 & 0.77 \\
\hline \multirow[t]{3}{*}{$7-4,25-28$} & Basalt & 51.2 & 13.8 & 3.85 & 8.24 & 6.67 & 11.5 & 2.24 & 0.24 & 0.72 & 0.04 & 1.06 & 0.34 & 0.10 & 100.00 & 0.72 \\
\hline & Mean & 51.2 & 13.8 & 4.68 & 7.63 & 6.65 & 11.5 & 2.22 & 0.26 & 0.74 & 0.04 & 1.07 & 0.32 & 0.08 & 100.19 & 0.74 \\
\hline & Range & 0.7 & 0.4 & 2.49 & 1.37 & 0.12 & 0.3 & 0.14 & 0.15 & 0.05 & 0.04 & 0.02 & 0.04 & 0.03 & 0.33 & 0.05 \\
\hline
\end{tabular}

a Analysts: Dawn Hutchison, A. E. Davis and K. A. Holmes.

${ }^{b}$ Results expressed on a moisture-free basis.

Table 13. Chemical analyses ${ }^{a}$ of basalts, a tuff and olivine-dolerites from Hole 555A (wt.\%).

\begin{tabular}{|c|c|c|c|c|c|c|c|c|c|c|c|c|c|c|c|c|}
\hline $\begin{array}{l}\text { Core-Section } \\
\text { (interval in } \mathrm{cm} \text { ) }\end{array}$ & Lithology & $\mathrm{SiO}_{2}$ & $\mathrm{Al}_{2} \mathrm{O}_{3}$ & $\mathrm{Fe}_{2} \mathrm{O}_{3}$ & $\mathrm{FeO}$ & $\mathrm{MgO}$ & $\mathrm{CaO}$ & $\mathrm{Na}_{2} \mathrm{O}$ & $\mathrm{K}_{2} \mathrm{O}$ & $\mathrm{H}_{2} \mathrm{O}^{+}$ & $\mathrm{CO}_{2}$ & $\mathrm{TiO}_{2}$ & $\mathrm{MnO}$ & $\mathrm{P}_{2} \mathrm{O}_{5}$ & Total & $\mathrm{H}_{2} \mathrm{O}^{-}$ \\
\hline $69-2,113-116$ & Basalt & 49.9 & 14.0 & 3.82 & 8.61 & 5.96 & 11.4 & 2.60 & 0.11 & 1.40 & 0.26 & 1.68 & 0.42 & 0.14 & 100.30 & 1.33 \\
\hline $77-4,74-77$ & Tuff & 47.1 & 12.4 & 9.66 & 3.22 & 9.49 & 2.8 & 4.82 & 0.12 & 8.86 & 0.34 & 1.26 & 0.26 & 0.08 & 100.41 & 9.20 \\
\hline $90-1,125-128$ & Basalt & 49.5 & 13.2 & 5.81 & 7.20 & 6.99 & 10.9 & 2.42 & 0.07 & 2.13 & 0.00 & 1.34 & 0.39 & 0.08 & 100.03 & 2.26 \\
\hline $95-1,40-43$ & Olivine-dolerite & 49.7 & 13.9 & 2.82 & 8.79 & 7.50 & 12.5 & 2.10 & 0.09 & 1.19 & 0.10 & 1.08 & 0.34 & 0.08 & 99.49 & 0.56 \\
\hline $96-2,95-98$ & Olivine-dolerite & 49.6 & 13.8 & 3.62 & 8.22 & 7.25 & 12.4 & 2.11 & 0.09 & 1.37 & 0.00 & 1.15 & 0.40 & 0.08 & 100.09 & 0.66 \\
\hline $97-3,36-39$ & Olivine-dolerite & 50.3 & 13.7 & 4.16 & 8.23 & 6.31 & 12.2 & 2.50 & 0.05 & 1.15 & 0.06 & 1.42 & 0.34 & 0.08 & 100.50 & 1.17 \\
\hline $97-6,80-83$ & Olivine-dolerite & 49.6 & 14.2 & 3.43 & 7.70 & 7.74 & 12.6 & 2.13 & 0.05 & 1.62 & 0.05 & 1.01 & 0.35 & 0.07 & 100.70 & 1.18 \\
\hline \multirow[t]{3}{*}{$96-5,108-110$} & Olivine-dolerite & 49.6 & 14.1 & 3.26 & 8.16 & 7.76 & 12.5 & 2.18 & 0.06 & 2.12 & 0.00 & 1.07 & 0.39 & 0.06 & 101.26 & 0.94 \\
\hline & Mean ${ }^{c}$ & 49.7 & 13.8 & 3.85 & 8.13 & 7.07 & 12.1 & 2.29 & 0.09 & 1.57 & 0.05 & 1.25 & 0.36 & 0.08 & 100.35 & 1.16 \\
\hline & Range $^{c}$ & 0.8 & $\begin{array}{l}1.0 \\
1.0\end{array}$ & 2.55 & $\begin{array}{l}0.13 \\
1.59\end{array}$ & 1.80 & 1.7 & 0.50 & 0.07 & 0.98 & 0.26 & 0.57 & 0.07 & 0.08 & 1.77 & 1.60 \\
\hline
\end{tabular}

a Analysts: Dawn Hutchison, A. E. Davis and K. A. Holmes.

besults expressed on a moisture-free basis.

${ }^{c}$ Excluding the tuff sample.

tain higher $\mathrm{CaO}$ on average than all other basalts but otherwise are closely similar chemically.

The vitroclastic tuff sample from Hole 553A has markedly higher $\mathrm{Fe}_{2} \mathrm{O}_{3}, \mathrm{H}_{2} \mathrm{O}^{+}$, and $\mathrm{K}_{2} \mathrm{O}$, and lower $\mathrm{FeO}, \mathrm{CaO}$, $\mathrm{MgO}, \mathrm{P}_{2} \mathrm{O}_{5}$, and $\mathrm{MnO}$ compared with basalts. However, $\mathrm{SiO}_{2}, \mathrm{Al}_{2} \mathrm{O}_{3}, \mathrm{Na}_{2} \mathrm{O}$, and $\mathrm{TiO}_{2}$ are similar. The tuff from Hole 555 is similarly enriched and depleted relative to the basalts, except that $\mathrm{Na}_{2} \mathrm{O}$ is here greater in the tuff and $\mathrm{P}_{2} \mathrm{O}_{5}$ similar to that of the basalts.

Plotted on a $\mathrm{SiO}_{2}$-alkali diagram (Fig. 2), all of the analyzed basalts and dolerites fall in the subalkaline basalt field of Irvine and Baragar (1971) ("tholeiitic" series of Kuno, 1960). One high- $\mathrm{SiO}_{2}$ sample (Sample $553 \mathrm{~A}-40-1,79-82 \mathrm{~cm}$ ) from Hole 553A falls in the andesite range of the proposed classification of the IUGS (basalt: andesite limit at $\mathbf{5 2 \%} \mathrm{SiO}_{2}$ ). There is little systematic variation of alkalies with $\mathrm{SiO}_{2}$, with between 2 and $3 \%\left(\mathrm{Na}_{2} \mathrm{O}+\mathrm{K}_{2} \mathrm{O}\right)$. In this range also lie the $\mathrm{SiO}_{2}-$ poorer, mainly subaerial, Blosseville Coast basalts of East Greenland, from the rifted counterpart of the Greenland-Rockall continent (Brooks et al., 1976) and the opposing Faeroes basalts (Bollingberg et al., 1975). A tuff from Hole 555 lies in the alkaline basalt field whereas that from Hole 553A lies at the basalt/andesite boundary.
Plots of the major oxides on the solidification index (SI) variation diagram (Fig. 3 ) reveal some positive and negative linear correlations which suggest that at some sites the flows are related by fractionation from a common parent magma. This is clearly displayed by basalts from Hole 553A which show negative linear correlation for $\mathrm{FeO}, \mathrm{MnO}, \mathrm{Na}_{2} \mathrm{O}, \mathrm{TiO}_{2}$, and $\mathrm{P}_{2} \mathrm{O}_{5}$, and correspondingly positive linear correlation for $\mathrm{MgO}$. Poor correlation between $\mathrm{SI}$ and $\mathrm{CaO}$ or $\mathrm{Al}_{2} \mathrm{O}_{3}$ may reflect the widespread occurrence of xenocrystic plagioclase in the Hole $553 \mathrm{~A}$ basalts. The $\mathrm{K}_{2} \mathrm{O}$-deficient nature of these basalts is emphasized by the barely discernible negative correlation with SI. Similar trends were obtained by combining plots of Hole 555 basalts and dolerites, suggesting that the two rock types are, respectively, more and less fractionated portions of the same parental magma. The small group of basalts from Hole 554A possess higher $\mathrm{SiO}_{2}$ values, higher $\mathrm{K}_{2} \mathrm{O}$, and generally show anomalous correlations with SI compared to the other basalt groups. Figures 4 and 5 illustrate the negative linear correlation between $\mathrm{MgO}$ and both alkalis and $\mathrm{TiO}_{2}$ for all the basalts analyzed.

In the FMA diagram (Fig. 6), the Leg 81 basaltic rocks cluster closely in the $\mathrm{FeO} 45-60 \%$ range $\left(\mathrm{Na}_{2} \mathrm{O}+\right.$ $\mathrm{K}_{2} \mathrm{O}$ ) $10-20 \%$, $\mathrm{MgO} 30-46 \%$; they show some align- 


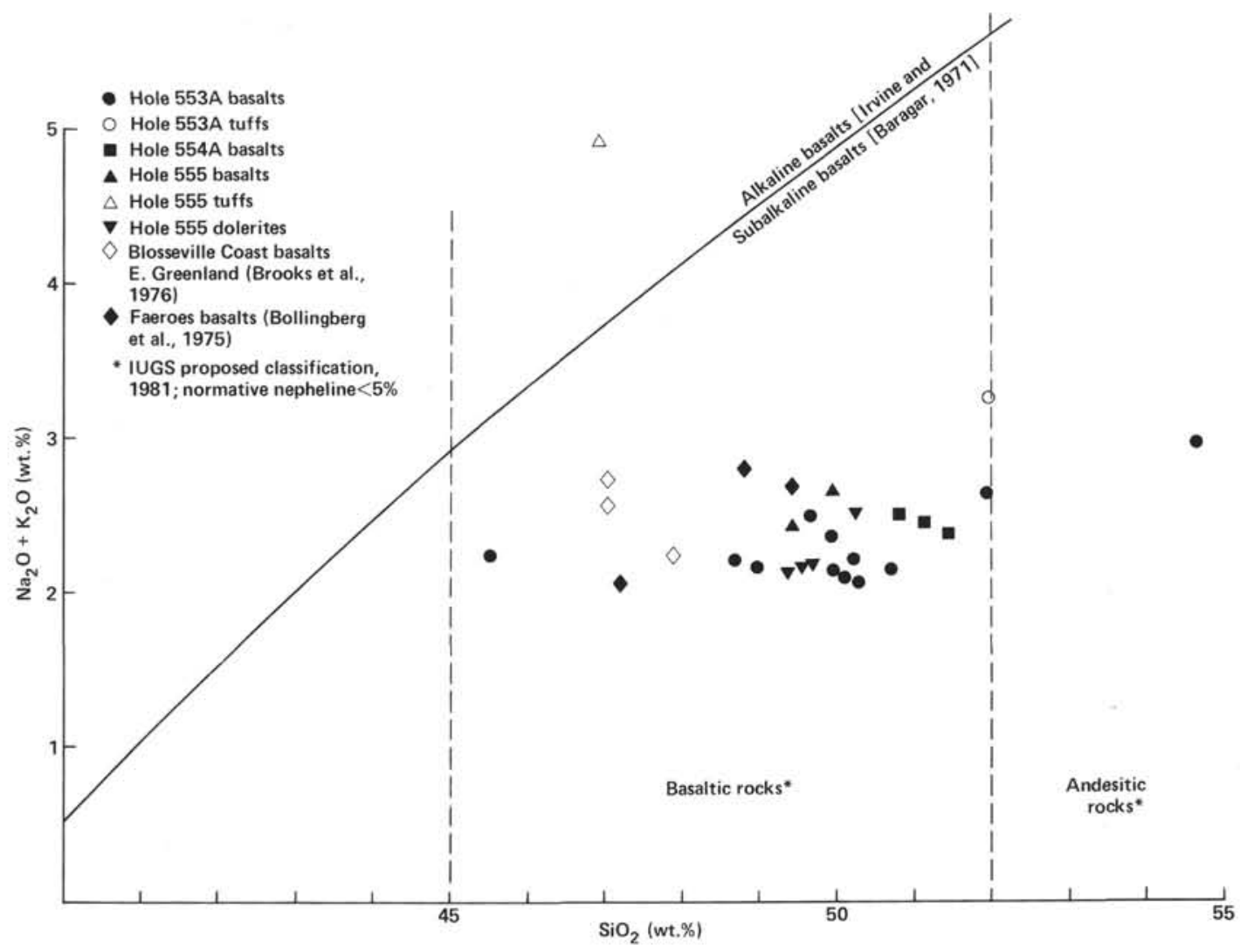

Figure 2. Alkali-silica variation diagram for basalts from Holes 553A, 554A, and 555 and for dolerites from Hole 555.

ment along the Fe-enrichment trend of the "tholeiite" type. Compared with Leg 49 basalts from the Mid-Atlantic (Reykjanes) Ridge (Wood et al., 1979), the present basalts lie mainly in their field. There is little apparent difference between samples from Sites 553, 554, and 555 . (See Figs. 7, 8.)

\section{Norms}

CIPW norms based on the full analyses are listed in Table 14 (Hole 553A basalts) and Table 15 (Hole 554A and 555 dolerite) together with agpaicity coefficients (Na $+\mathrm{K}$ )/ $\mathrm{Al}$ and differentiation indices (sums of weight percent normative salic minerals). Although the analyzed basalts are the least altered of all the samples examined, with $\mathrm{Fe}_{2} \mathrm{O}_{3}$ less than $7.0 \%$ and $\left(\mathrm{H}_{2} \mathrm{O}^{+}+\mathrm{CO}_{2}\right)$ contents of mainly less than $5 \%$, nevertheless all contain minor alteration products-smectite and celadonite; therefore, the norms must be treated with some caution. Allowing for this alteration, however, basalts are quartz normative (the dolerites of Hole 555 only slightly so), and strongly hypersthene normative, thus substantiating their tholeiitic character. No normative olivine occurs in the calculation, although sporadically minor to trace modal olivine occurs in some samples.

\section{Trace- and Minor-Element Geochemistry}

In order to ascertain the general levels of trace elements in basalts and volcaniclastic rocks of Holes 553A, $554 \mathrm{~A}$, and 555, their intra- and interhole and interflow variations, analyses were made in duplicate for 21 trace elements by direct-reader emission spectrography. ${ }^{4} \mathrm{We}$ list all results in Tables 16-18 and a summary of means and ranges in Table 19. Average detection limits are (in ppm) : Li, 0.4; Rb, 2.5; Cu, 0.7; Ag, 0.8; $\mathrm{Be}, 0.5 ; \mathrm{Sr}, 1$; $\mathrm{Zn}, 12$; B, 7; Ga, 6; Y, 7; La, 30; Sn, 6; Pb, 20; Zr, 30; $\mathrm{Bi}, 7$;, 9 ; $\mathrm{Nb}, 14$; $\mathrm{Cr}, 7$; Mo, 3; $\mathrm{Co}, 6$; Ni, 7. Rare earths-La, Ce, Nd, Sm, Eu, Tb, Ho, Yb, and $\mathrm{Lu}-$ determined on seven samples by Dr. Susan Parry, University of London Reactor Center, are listed in Table 20, and chondrite-normalized values plotted in Figure 9.

\section{Interflow Variation: Hole 553A}

\section{Alkali Metals, Group IA: Li, Rb}

$\mathrm{Na}$ and $\mathrm{K}$ show, on the whole, little variation within the basalt lava flows, but more variation is shown by $\mathrm{Li}$ and $\mathrm{Rb}$. $\mathrm{Li}$ range: $0.0-9.3$ (mean 5.32, s.d. 1.39 ), $\mathrm{Rb}$ range: $0.0-24.5$ (mean 1.87 , s.d. 4.12 ). However some flow tops and particularly flow breccias and pyroclastic rocks contain above average $\mathrm{Li}$ (mean 10.06 , s.d. 6.76) and $\mathrm{Rb}$ (mean 11.79, s.d. 6.66), but these variations are not systematic. Because of extensive alteration (mainly to smectite) it is not possible to attribute the higher values to any one primary phase. $\mathrm{Li}$ is partitioned between, inter alia, micas and pyroxenes during the crystallization of a magma. In weathering, $\mathrm{Li}$ and $\mathrm{Rb}$ are incorporated in hydrolyzates, thus explaining the high values in

\footnotetext{
${ }^{4}$ Analysts: K. A. Holmes, P. Joseph, and V. A. Judge, Analytical Chemistry Unit, Institute of Geological Sciences, London.
} 


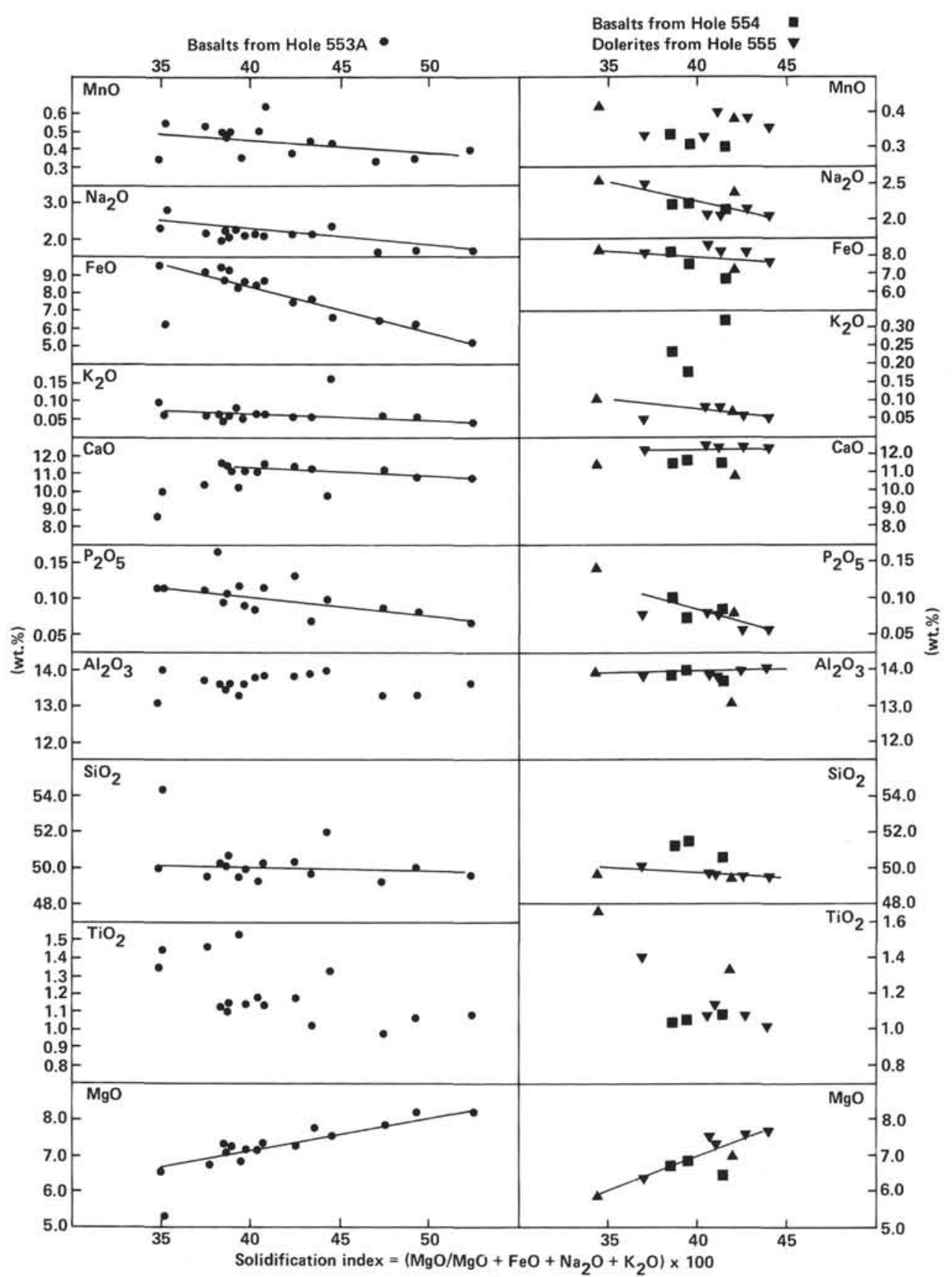

Figure 3. Variation diagram for major oxides versus solidification index $(\mathrm{SI}=\mathrm{MgO} / \mathrm{MgO}+\mathrm{FeO}+$ $\mathrm{Na}_{2} \mathrm{O}+\mathrm{K}_{2} \mathrm{O}$ ) for basalts from Holes 553A, 554A, 555 and for dolerites from Hole 555.

the argillized volcaniclastics. Although published data for $\mathrm{Li}$ in seafloor basalts are very sparse, most $\mathrm{Rb}$ values in Hole $553 \mathrm{~A}$ basalts are of similar range to these, for example, from the Franco-American Mid Ocean Undersea Study (FAMOUS) area (Roex et al., 1981), or normal mid-ocean ridge basalts (MORB) $\left(22-25^{\circ} \mathrm{N}\right)$ (Bryan et al., 1981), or from International Phase of Ocean Drilling (IPOD) Leg 49 (Wood et al., 1979).

\section{Group 1B: $\mathrm{Cu}, \mathrm{Ag}$}

There is no systematic variation of these with depth, position in flows, lithology, or mineralogy. The $\mathrm{Cu}$ values are considerably higher than those from the FAMOUS area (Roex et al., 1981) and from "normal MORB" $\left(22-25^{\circ} \mathrm{N}\right)$ (Bryan et al., 1981). No data for $\mathrm{Ag}$ are available for comparison, although the levels are extremely low.

\section{Group IIA: Be, $\mathrm{Sr}$}

The Be contents are all extremely low, and no significance is attached to their apparent variation. Sr values are very constant throughout the basalts, but a little lower in some of the volcaniclastic rocks. Because plagioclase (the main host of $\mathrm{Sr}$ ) remains little altered in even the most heavily smectitized basalt, the contents of $\mathrm{Sr}$ do not vary with alteration. There is no apparent var- 


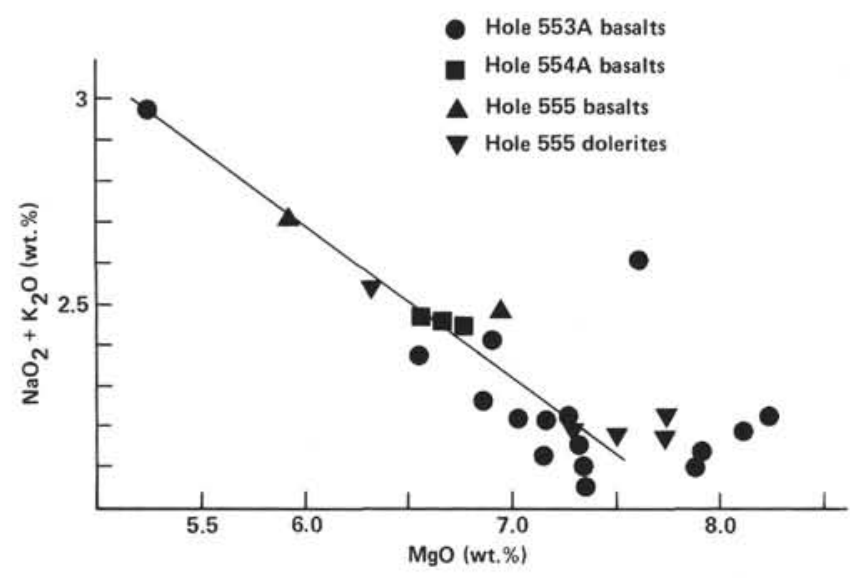

Figure 4. Alkali: $\mathrm{MgO}$ diagram for basalts from Holes 553A, 554A, and 555 and for dolerites from Hole 555 .

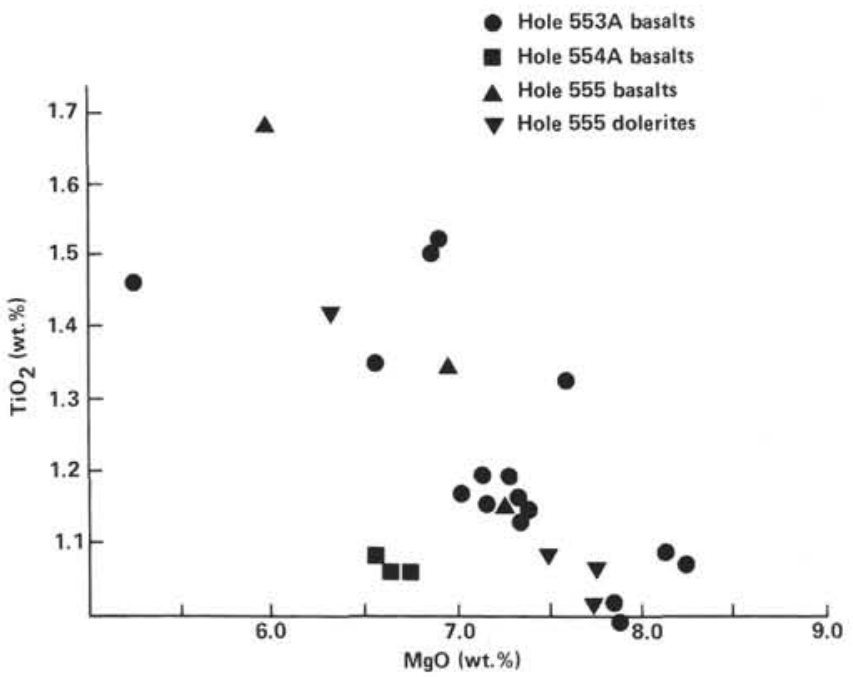

Figure 5. $\mathrm{TiO}_{2}: \mathrm{MgO}$ diagram for basalts from Holes 553A, 554A, and 555 and for dolerites from Hole 555 .

iation with position in flow. The basalt $\mathrm{Sr}$ values are slightly lower than for normal MORB $\left(22-25^{\circ} \mathrm{N}\right)$ and for basalts from the FAMOUS area but fall in the range of Mid-Atlantic Ridge (MAR) basalts from the Central North Atlantic (White and Schilling, 1978).

\section{Group IIB: $\mathrm{Zn}$}

This shows no systematic variation throughout the samples. The range is similar to that for normal MORB and seafloor basalts elsewhere (e.g., IPOD Leg 49).

\section{Group IIIA: B, Ga}

Boron, being an oxyphile, is commonly enriched in volcanic emanations. The highest values here occur in the volcaniclastic rocks (53.3-60.0 ppm) in the upper sequence. But other relatively high values occur sporadically in the basalts, some (but not all) relating to flow tops. Gallium, on weathering, tends to concentrate in hydrolyzates. However, the values are relatively constant irrespective of position in flows, although perhaps slightly lower in the volcaniclastics.

\section{Group IIIB: $Y, L a$}

Values of Y vary relatively little throughout the sequence. The highest value (100.2 ppm) relates to an agglomeratic basalt; other peaks relate to vitroclastic tuffs or basalts, irrespective of lithology or flow position. The mean Y content (31.32, s.d. 11.65) lies in the range for IPOD Leg 49 basalts. La values are sporadic (mean 3.41, s.d. 4.6) without systematic variation with Y. In view of the low La results no inferences can be drawn; the mean lies in the range for normal MORB $\left(22-25^{\circ} \mathrm{N}\right)$; the element is discussed below in the context of the rare earth suite. In the discriminant plot of $\mathrm{Y}(\times 3)$ versus $\mathrm{Ti}(\div 100)$ versus $\mathrm{Zr}$ (Fig. 10), the Hole 553A basalts plot mainly in the ocean-floor basalt and calc-alkali basalt fields, with scatter to the within-plate basalt field.

\section{Group IVA: $\mathrm{Sn}, \mathrm{Pb}$}

The Sn values vary little at very low levels throughout the sequence (mean 4.68, s.d. 1.78). The $\mathrm{Pb}$ values are also very low (mean 2.73, s.d. 4.48); the highest Sn contents appear to relate to some higher volcaniclastic rocks as well as to some tops of flows, but there is no systematic relationship. There is a sparsity of data on Sn and $\mathrm{Pb}$ in seafloor basalts for comparison.

\section{Group IVB: $\mathrm{Zr}$}

Values vary relatively little throughout the sequence (mean 112, s.d. 29.7), and there is no relationship to lithology, position in flow, or alteration. The values follow those for normal MORB $\left(22-25^{\circ} \mathrm{N}\right)$, and for IPOD Leg 49 basalts, but are mainly twice those for FAMOUS basalts. There is a positive correlation between $\mathrm{Zr}$ and $\mathrm{Y}$ (Fig. 11), but none between $\mathrm{Zr}$ and $\mathrm{Nb}$, which are alleged to covary in cogenetic suites (Erlank and Kable, 1976).

\section{Group VA: Bi}

The values reported are all very low and there is no relationship between the sporadic variation and lithology or other factors.

\section{Group VB:, $\mathrm{Nb}$}

$\mathrm{V}$ and $\mathrm{Nb}$ show positive correlation (Fig. 12) as expected geochemically. There is no correlation between $\mathrm{V}$ and $\mathrm{Ti}$, although $\mathrm{V}$ is commonly enriched in $\mathrm{Ti}-\mathrm{Fe}$ oxides during initial crystallization of a magma. Group V is probably mainly held in the secondary hydrolyzates, although there is no apparent correlation between degree of basalt smectitization and V content. The mean (316, s.d. 65.6) falls in the range recorded for IPOD Leg 49 basalts and normal MORB $\left(22-25^{\circ} \mathrm{N}\right)$. The positive linear correlation with $\mathrm{Nb}$ suggests that it too is mainly held in the secondary clay minerals; in this respect the salts of $\mathrm{Nb}$ (and $\mathrm{Ta}$ ) are readily hydrolyzed during weathering (Rankama and Sahama, 1950).

\section{Group VIB: $\mathrm{Cr}$, Mo}

The Mo values are very low and variable (mean 1.4, s.d. 1.9). $\mathrm{Cr}$ (mean for basalts 83.95 , s.d. 56.23 ) is relatively enriched in the lowest samples (attaining $257 \mathrm{ppm}$ ) 


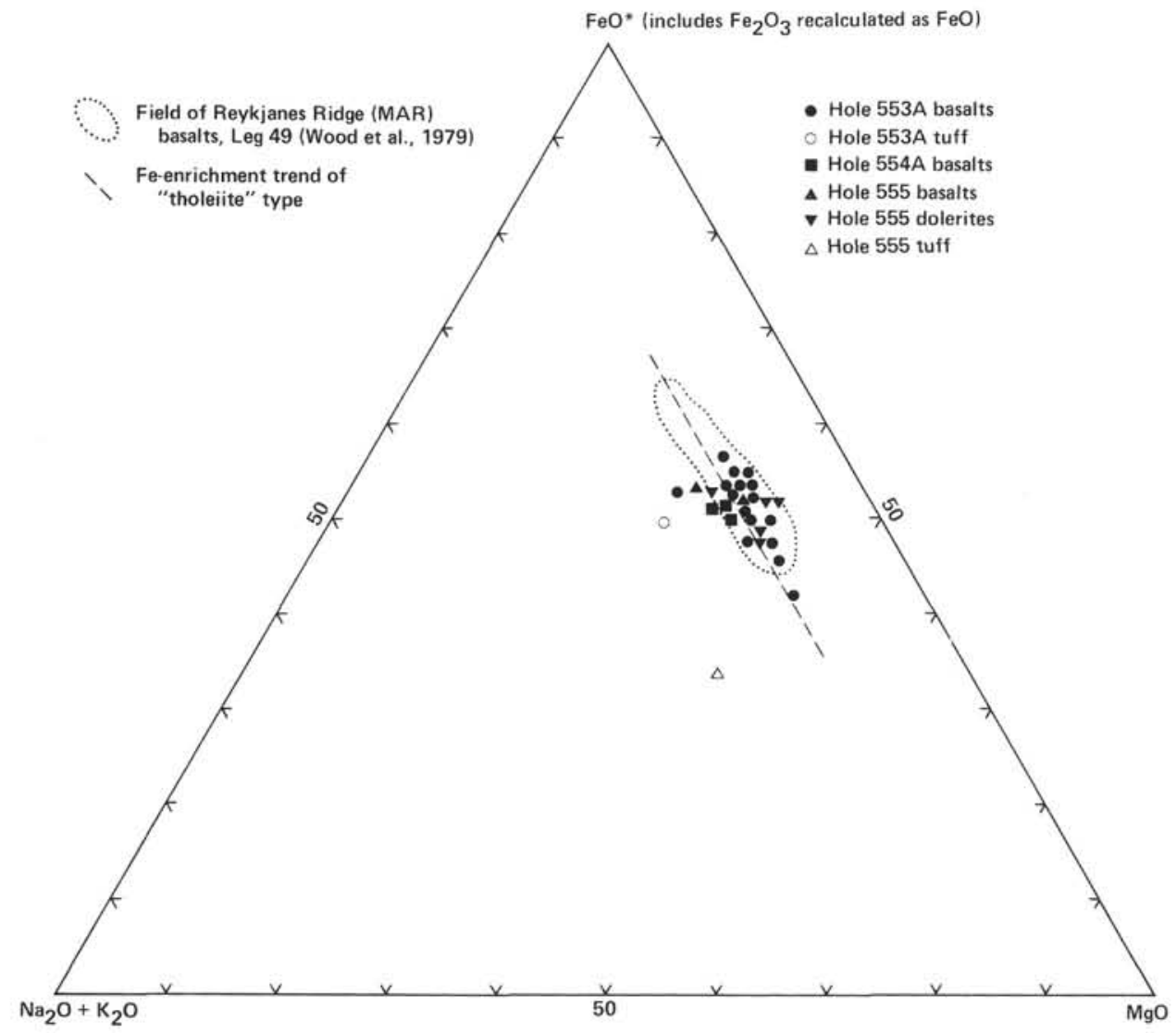

Figure 6. FMA diagram for basalts from Holes 553A, 554A, and 555 and for dolerites from Hole 555.

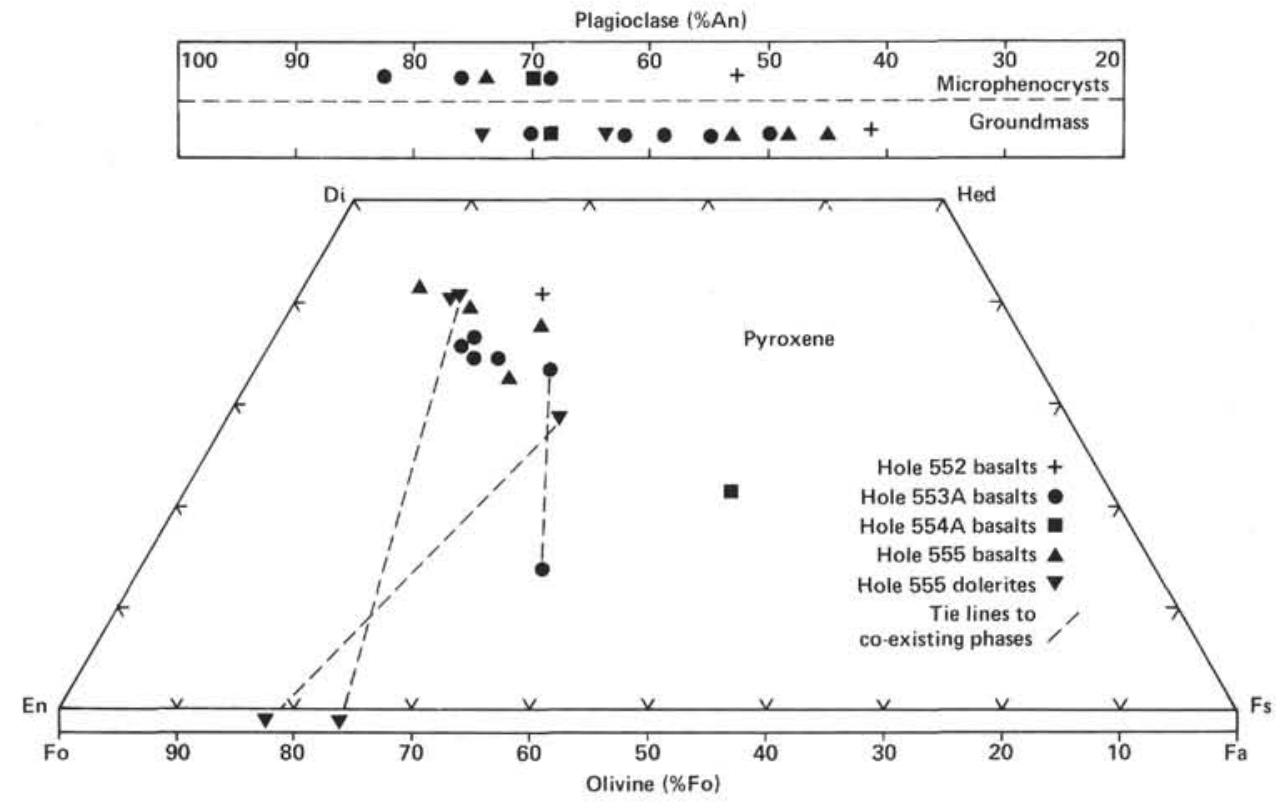

Figure 7. Composition of primary silicates in basalts from Holes 552, 553A, 554A, and 555 and in dolerites from Hole 555. 


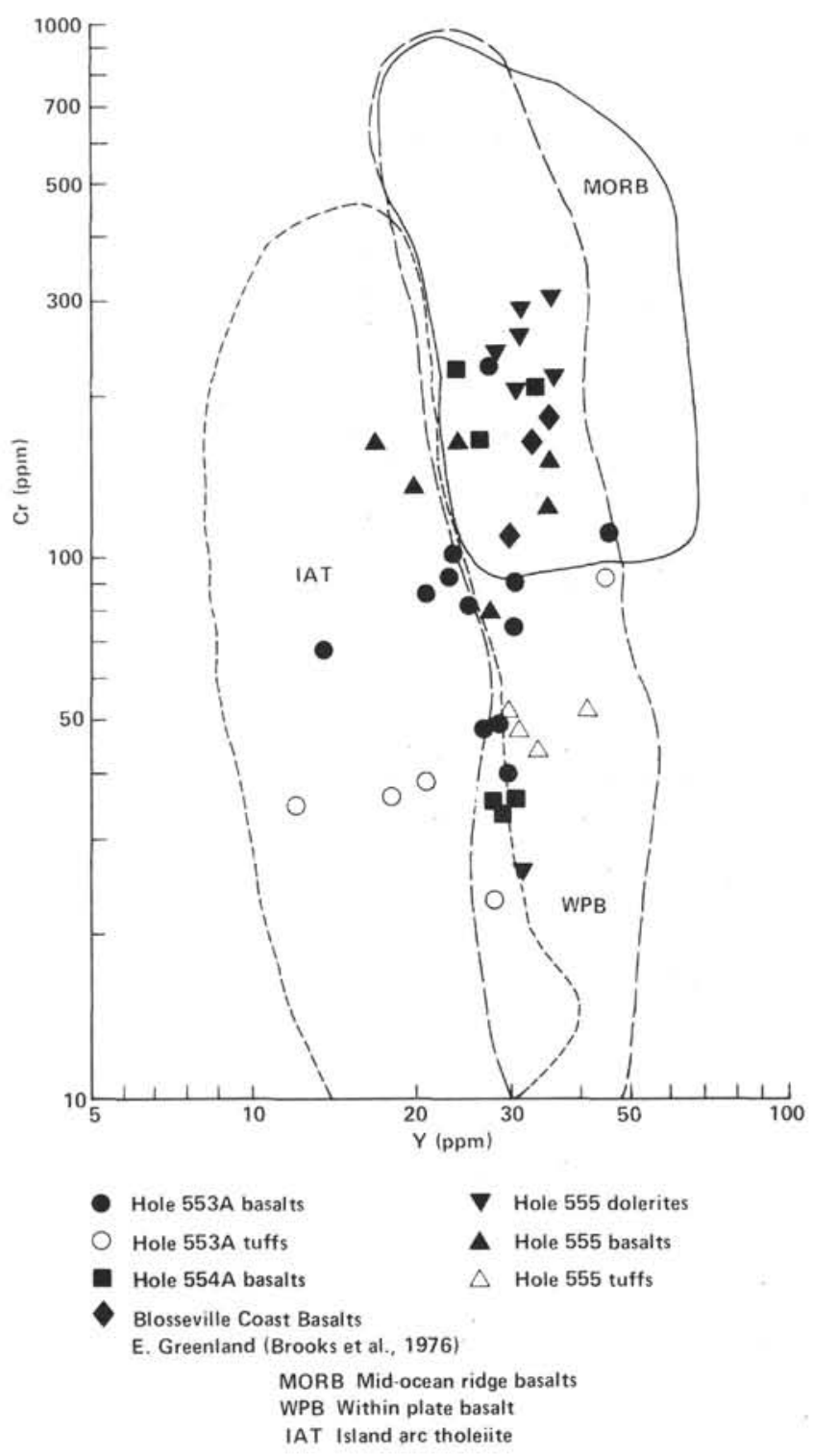

Figure 8. Discriminant diagram of $\mathrm{Cr}$ versus $\mathrm{Y}$ for basalts from Holes 553A, 554A, and 555; dolerites from Hole 555; and for East Greenland basalts.

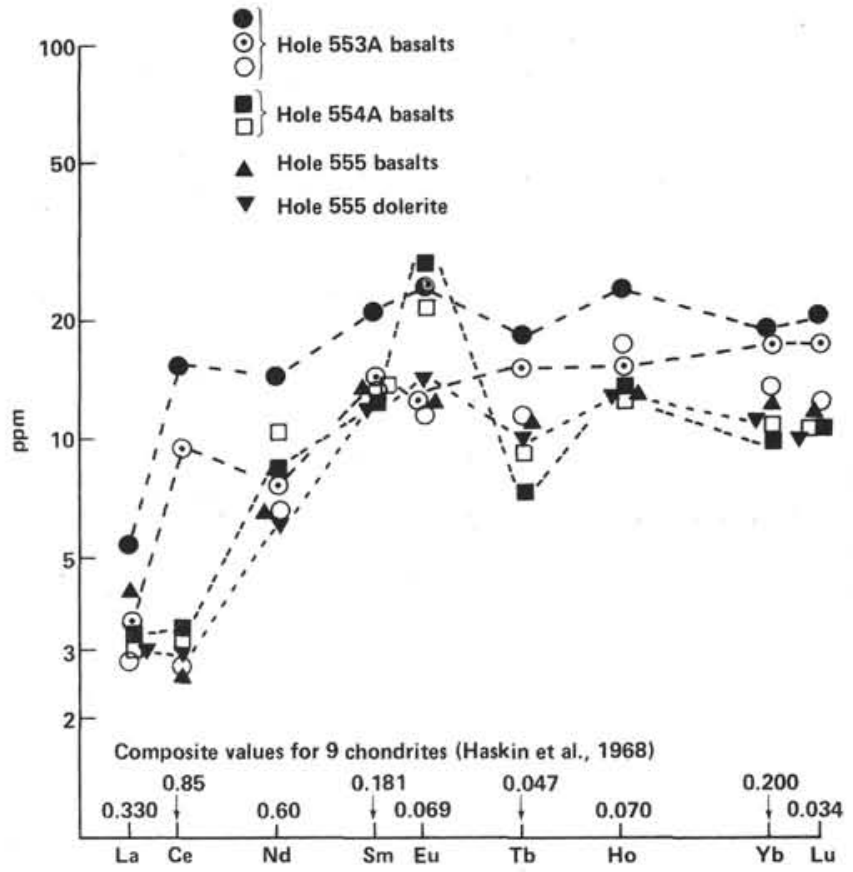

Figure 9. Chondrite-normalized REE patterns for basalts from Holes $553 \mathrm{~A}, 554 \mathrm{~A}$, and 555 and for a dolerite from Hole 555 .

of slightly or partly saponitized feldspar-phyric basalts. The lower values occur in the upper volcaniclastic rocks (down to $20.3 \mathrm{ppm}$ ). Cr generally correlates positively with $\mathrm{Ni}$; both elements tend to concentrate in hydrolyzates during weathering, but there is no apparent relationship in the present samples between $\mathrm{Cr}$ (and $\mathrm{Ni}$ ) and degree of argillization of the basalts. Apart from the basalt units noted, the $\mathrm{Cr}$ contents are considerably lower than those recorded for IPOD Leg 49 basalts, or from normal MORB $\left(22-25^{\circ} \mathrm{N}\right)$, but approach those of plagioclase-pyroxene basalts from the FAMOUS area.

\section{Group VIII: $\mathrm{Co}, \mathrm{Ni}$}

Although geochemically related, these elements behave differently in petrogenesis and alteration processes (Rankama and Sahama, 1950). However, the mean values are the same (117) in the basalts, although differ in the vitroclastic rocks (Co 85 ppm, s.d. 26.32; Ni 105 ppm,

Table 14. CIPW norms of basalts from Hole 553A, Leg 81 .

\begin{tabular}{|c|c|c|c|c|c|c|c|c|c|c|c|c|c|c|c|c|c|}
\hline & \multirow[b]{2}{*}{$\begin{array}{l}\text { Normative } \\
\text { mineral }\end{array}$} & \multicolumn{16}{|c|}{ Core-Section (interval in $\mathrm{cm}$ ) } \\
\hline & & $\begin{array}{c}38-2 \\
56-59\end{array}$ & $\begin{array}{l}40-1 \\
79-82\end{array}$ & $\begin{array}{l}45-5 \\
17-19\end{array}$ & $\begin{array}{c}46-1 \\
121-125\end{array}$ & $\begin{array}{l}46-3 \\
56-59\end{array}$ & $\begin{array}{l}46-4 \\
53-56\end{array}$ & $\begin{array}{l}47-3 \\
73-76\end{array}$ & $\begin{array}{l}48-2 \\
60-65\end{array}$ & $\begin{array}{l}51-1 \\
54-57\end{array}$ & $\begin{array}{c}51-2 \\
77-80\end{array}$ & $\begin{array}{l}53-1 \\
77-80\end{array}$ & $\begin{array}{l}54-4, \\
81-85\end{array}$ & $\begin{array}{l}55-5 \\
92-95\end{array}$ & $\begin{array}{l}55-6 \\
44-47\end{array}$ & $\begin{array}{c}56-1 \\
3-6\end{array}$ & $\begin{array}{c}59-2 \\
115-118\end{array}$ \\
\hline$Q$ & \multirow{4}{*}{$\mathrm{F}$} & 6.97 & 13.41 & 5.06 & 3.27 & 3.27 & 3.97 & 5.81 & 4.75 & 4.80 & 3.86 & 4.92 & 4.73 & 4.37 & 4.24 & 3.55 & 4.49 \\
\hline or) & & 0.93 & 0.59 & 0.36 & 0.30 & 0.35 & 0.36 & 0.48 & 0.36 & 0.36 & 0.36 & 0.36 & 0.36 & 0.30 & 0.36 & 0.36 & 0.24 \\
\hline ab & & 20.51 & 24.26 & 18.32 & 17.06 & 17.71 & 17.33 & 20.02 & 18.82 & 17.90 & 17.61 & 18.53 & 19.91 & 17.80 & 18.39 & 18.65 & 18.67 \\
\hline an & & 26.33 & 24.90 & 27.88 & $27: 80$ & 27.73 & 27.83 & 25.59 & 26.84 & 28.39 & 28.55 & 28.31 & 25.66 & 27.97 & 27.72 & 28.39 & 33.50 \\
\hline di? & \multirow{3}{*}{$P$} & 16.72 & 13.24 & 22.18 & 22.93 & 23.30 & 23.60 & 19.53 & 20.02 & 21.01 & 21.68 & 19.03 & 19.50 & 21.15 & 21.34 & 17.21 & 16.09 \\
\hline hy\} & & 16.22 & 10.86 & 15.60 & 20.86 & 19.91 & 18.45 & 16.10 & 18.53 & 15.92 & 19.36 & 17.38 & 19.61 & 19.38 & 19.21 & 21.66 & 17.21 \\
\hline $\mathrm{mt}$ & & 9.77 & 9.61 & 8.00 & 5.06 & 5.24 & 5.98 & 9.19 & 7.49 & 9.36 & 6.31 & 8.50 & 7.01 & 6.15 & 5.90 & 5.42 & 7.50 \\
\hline \multicolumn{2}{|l|}{ il } & 2.47 & 2.76 & 2.27 & 2.16 & 2.20 & 2.17 & 2.92 & 2.88 & 1.92 & 1.94 & 2.05 & 2.59 & 2.21 & 2.24 & 2.29 & 2.10 \\
\hline \multirow{2}{*}{\multicolumn{2}{|c|}{ ap }} & 0.23 & 0.32 & 0.30 & 0.40 & 0.25 & 0.28 & 0.28 & 0.28 & 0.19 & 0.16 & 0.19 & 0.28 & 0.19 & 0.21 & 0.19 & 0.17 \\
\hline & & 0.29 & 0.00 & 0.00 & 0.14 & 0.00 & 0.00 & 0.05 & 0.00 & 0.12 & 0.14 & 0.69 & 0.32 & 0.46 & 0.37 & 2.23 & 0.00 \\
\hline \multicolumn{2}{|c|}{$\begin{array}{l}\text { cc } \\
\text { Agpaicity coefficient }\end{array}$} & 0.30 & 0.35 & 0.26 & 0.25 & 0.26 & 0.25 & 0.30 & 0.27 & 0.25 & 0.25 & 0.26 & 0.30 & 0.26 & 0.26 & 0.26 & 0.23 \\
\hline \multicolumn{2}{|c|}{ Differentiation index } & 54.75 & 63.16 & 51.62 & 48.43 & 49.07 & 49.49 & 51.90 & 50.76 & 51.45 & 50.38 & 52.12 & 50.66 & 50.43 & 50.70 & 50.96 & 56.90 \\
\hline
\end{tabular}


Table 15. CIPW norms of basalts and dolerites from Holes 554A and 555A, Leg 81 .

\begin{tabular}{|c|c|c|c|c|c|c|c|c|c|c|}
\hline \multirow[b]{2}{*}{$\begin{array}{c}\text { Normative } \\
\text { mineral }\end{array}$} & \multicolumn{10}{|c|}{ Sample (interval in $\mathrm{cm}$ ) } \\
\hline & $\begin{array}{c}554 A-7-1 \\
63-66\end{array}$ & $\begin{array}{c}554 \mathrm{~A}-7-3, \\
10-12\end{array}$ & $\begin{array}{c}554 \mathrm{~A}-7-4 \\
25-28\end{array}$ & $\begin{array}{c}555 A-69-3 \\
113-116\end{array}$ & $\begin{array}{c}555 A-90-1 \\
125-128\end{array}$ & $\begin{array}{c}555 \mathrm{~A}-95-1 \\
97-100\end{array}$ & $\begin{array}{c}\text { 555A-96-2, } \\
95-98\end{array}$ & $\begin{array}{c}555 A-97-3, \\
36-39\end{array}$ & $\begin{array}{c}555 A-97-6 \\
80-83\end{array}$ & $\begin{array}{c}555 \mathrm{~A}-96-5 \\
108-110\end{array}$ \\
\hline Q & 6.72 & 4.68 & 4.47 & 3.33 & 4.72 & 0.82 & 2.01 & 3.14 & 1.20 & 0.53 \\
\hline or) & 2.02 & 1.13 & 1.43 & 0.66 & 0.42 & 0.54 & 0.54 & 0.30 & 0.30 & 0.36 \\
\hline $\mathrm{ab} \quad \mathrm{F}$ & 18.16 & 19.37 & 19.07 & 22.22 & 20.89 & 17.95 & 18.06 & 21.27 & 18.20 & 18.58 \\
\hline an & 26.61 & 27.56 & 27.09 & 26.50 & 25.49 & 28.55 & 28.28 & 26.19 & 29.36 & 28.76 \\
\hline di $p$ & 23.49 & 24.56 & 24.01 & 22.97 & 23.48 & 27.44 & 27.25 & 27.55 & 26.78 & 27.13 \\
\hline hy $P$ & 11.35 & 14.82 & 15.92 & 14.54 & 13.57 & 18.28 & 16.11 & 12.41 & 16.90 & 17.65 \\
\hline $\mathrm{mt}$ & 9.23 & 5.61 & 5.62 & 5.60 & 8.61 & 4.14 & 5.32 & 6.07 & 5.03 & 4.77 \\
\hline il & 2.06 & 2.02 & 2.03 & 3.23 & 2.60 & 2.07 & 2.21 & 2.72 & 1.94 & 2.05 \\
\hline ap & 0.19 & 0.16 & 0.23 & 0.33 & 0.19 & 0.19 & 0.19 & 0.19 & 0.16 & 0.14 \\
\hline cc & 0.14 & 0.05 & 0.09 & 0.60 & 0.00 & 0.00 & 0.00 & 0.14 & 0.11 & 0.00 \\
\hline Agpaicity coefficient & 0.29 & 0.28 & 0.29 & 0.31 & 0.31 & 0.26 & 0.26 & 0.30 & 0.25 & 0.26 \\
\hline Differentiation index & 53.51 & 52.74 & 52.06 & 52.70 & 51.52 & 47.86 & 48.89 & 50.90 & 49.05 & 48.24 \\
\hline
\end{tabular}

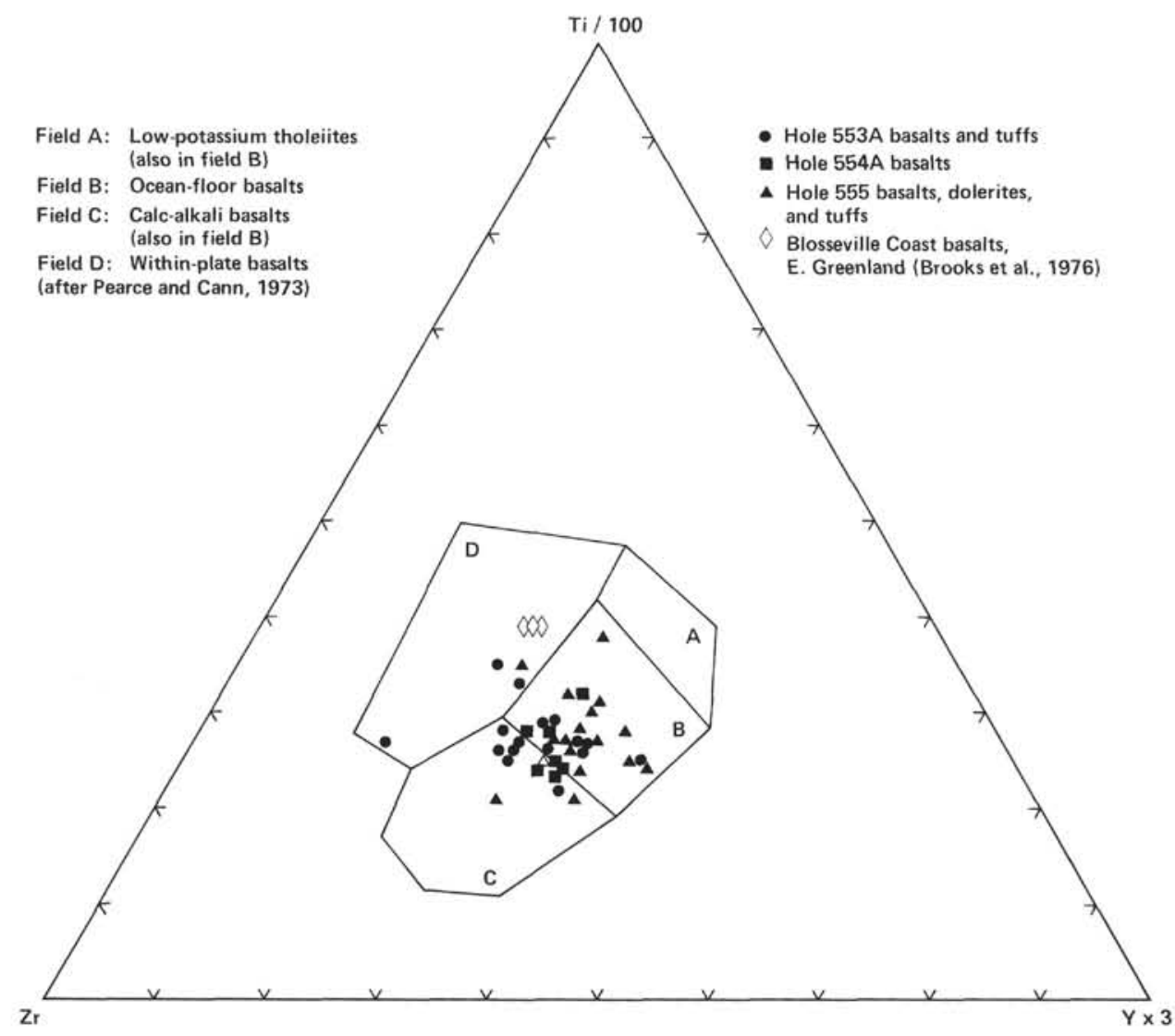

Figure 10. $\mathrm{Zr}: \mathrm{Ti} / 100: \mathrm{Y} \times 3$ diagram for basalts and tuffs from Hole $553 \mathrm{~A}$, basalts from Hole $554 \mathrm{~A}$, and of basalts, dolerites, and tuffs from Hole 555 .

s.d. 39.55). Similar enrichment and depletion with depth are shown between $\mathrm{Co}$ and $\mathrm{Ni}$, although the lowest basalts have low Co contents. The Co values are approximately twice those of other seafloor basalts-of IPOD Leg 49, of the FAMOUS area, and of normal MORB $\left(22-25^{\circ} \mathrm{N}\right)$. The $\mathrm{Ni}$ results fall in the general ranges of those seafloor basalts.

\section{Interflow Variation: Hole 554A}

Six samples only were available for analysis and since these are lithologically closely similar basalts, little sig- nificance can be deduced from the trace-element data (Table 17). The mean values for $\mathrm{Li}$ and $\mathrm{Rb}$ are more than twice those of Hole 553A basalts (Tables 16, 17). In this respect, $\mathrm{K}_{2} \mathrm{O}$ contents are also much higher than in the latter. Boron is considerably higher in the Hole 554A basalts (approaching the vitroclastic rocks of the other sites), suggesting much interaction with seawater or other supply of B. Other trace elements (except $\mathrm{Pb}$ ) are similar to those of Hole 553A basalts; thus the comparisons with published data on seafloor basalts noted above relate also to Site 554 basalts. 


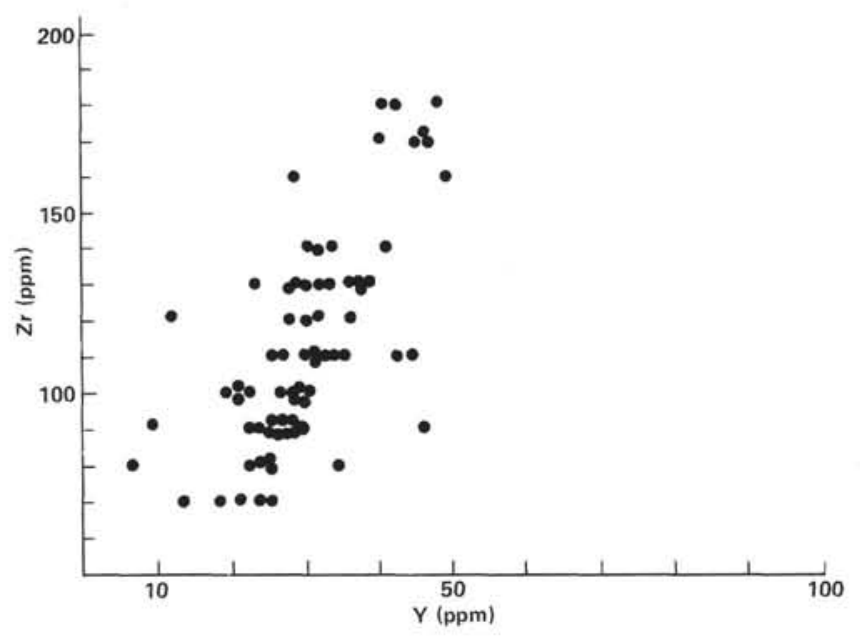

Figure 11. $\mathrm{Zr}$ :Y diagram for basalts from Hole 553A.

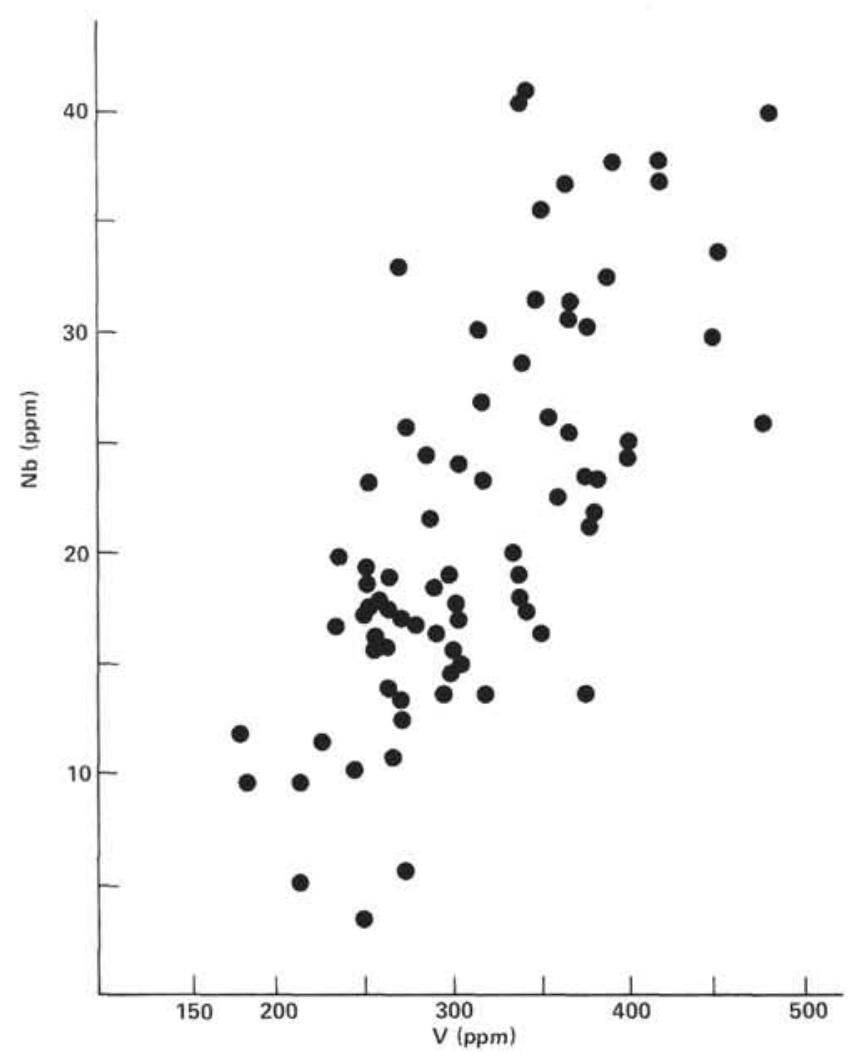

Figure 12. $\mathrm{Nb}: \mathrm{V}$ diagram for basalts from Hole 553A.

\section{Interflow Variations: Hole 555 and Comparisons}

\section{$\mathrm{Li}, \boldsymbol{R b}$}

There is a general correspondence between high- $\mathrm{Li}$ and volcaniclastic rocks and with some highly altered (saponitized) basalts; this enrichment is attributed to incorporation in the main alteration products-saponite and celadonite. Low $\mathrm{Li}$ values relate especially to the olivine-dolerites of Unit Vc, which, significantly, are little altered. The $\mathrm{Rb}$ data are more erratic, although the high- est value $(26.6 \mathrm{ppm})$ is for a saponitized lapilli tuff, while the olivine-dolerites all show low values (less than $2.9 \mathrm{ppm} \mathrm{Rb}$ ). The mean Li content of Hole 553A basalts (5.32) is, however, a little lower than that (6.0) for the fresh olivine-dolerites of Hole 555. Both alkali metals are relatively enriched in the volcaniclastic rocks of the two holes. Basalts of Hole 554A are more enriched in $\mathrm{Li}$ and $\mathrm{Rb}$ than the basalts of the other two holes, although the former are, on average, no more highly saponitized.

\section{$\mathrm{Cu}, \mathrm{Ag}$}

There is little significant variation in $\mathrm{Cu}$, although the lowest values occur in some of the volcaniclastic rocks. The mean $\mathrm{Cu}$ content for the basalts (232.6) is a little higher than that for Hole 553A basalts and distinctly higher than for Site 554 basalts. The $\mathrm{Cu}$ contents of Hole 555 basalts and olivine-dolerites are similar; saponitization appears to have little general effect on $\mathrm{Cu}$ contents. The volcaniclastics of Holes $553 \mathrm{~A}$ and 555 have closely similar mean $\mathrm{Cu}$ contents, which are still appreciably higher than for Hole 554A basalts. $\mathrm{Ag}$ is mainly below the limit of detection in Hole 555 .

\section{$\mathrm{Be}, \mathrm{Sr}$}

The Be contents are all low and there is no significant variation with lithology or other factor. The mean $\mathrm{Sr}$ content (103.3 ppm) for the basalts is a little higher than for Holes 553A and 554A basalts. The $\mathrm{Sr}$ values for the volcaniclastics are lower than for the basalts but are similar to those of Hole 553A; those for the olivine-dolerites are closely similar to the volcaniclastics and alteration has not appreciably affected the Sr contents.

\section{$\mathrm{Zn}$}

$\mathrm{Zn}$ contents range widely in the samples; there is no discernible variation with lithology, petrography, or degree of alteration. The means are close to those of the other two sites.

\section{$B, G a$}

The highest B values occur in the volcaniclastic rocks with one exception. Some very saponitized basalts are also enriched in B. The mean B content is close to that of the volcaniclastic rocks of Hole 553A. The mean B content of the Hole 555 basalts is also close to that of the basalts of Hole $553 \mathrm{~A}$, but both are considerably lower than that of Hole 554A basalts. The olivine-dolerites of Hole 555 have higher B contents than the basalts. Ga shows little variation irrespective of lithology at Site 555 (in common with the analyzed rocks from Holes 553A and 554A).

\section{$\mathrm{Sn}, \mathrm{Pb}$}

The Sn contents are very low and within the limits of error, apparently erratic without any relation to petrography, degree of alteration, or position within flows. The values of $\mathrm{Sn}$ are of similar order to those of the other sites. The $\mathrm{Pb}$ values, however, show a marked enrichment in the olivine-dolerite (Unit Vc), whereas the 
Table 16. Trace elements of basalts and pyroclastic rocks from Hole 553A (ppm).

\begin{tabular}{|c|c|c|c|c|c|c|c|c|c|c|c|c|c|c|c|c|c|c|c|c|c|c|c|}
\hline \multirow{2}{*}{$\begin{array}{l}\text { Core-Section } \\
\text { (interval in } \mathrm{cm} \text { ) }\end{array}$} & \multirow[b]{2}{*}{ Brief lithology } & \multirow{2}{*}{$\begin{array}{l}\text { Flow } \\
\text { position }\end{array}$} & \multicolumn{2}{|c|}{ IA } & \multicolumn{2}{|c|}{ IB } & \multicolumn{2}{|c|}{ IIA } & \multirow{2}{*}{$\frac{\text { IIB }}{\mathrm{Zn}}$} & \multicolumn{2}{|c|}{ IIIA } & \multicolumn{2}{|c|}{ IIIB } & \multicolumn{2}{|c|}{ IVA } & \multirow{2}{*}{$\frac{\text { IVB }}{\mathrm{Zr}}$} & \multirow{2}{*}{$\frac{\mathrm{VA}}{\mathrm{Bi}}$} & \multicolumn{2}{|c|}{ VB } & \multicolumn{2}{|c|}{ VIB } & \multicolumn{2}{|c|}{ VIII } \\
\hline & & & $\mathrm{Li}$ & $\mathrm{Rb}$ & $\mathrm{Cu}$ & $\mathrm{Ag}$ & $\mathrm{Be}$ & $\mathrm{Sr}$ & & B & $\mathrm{Ga}$ & $\mathrm{Y}$ & $\mathrm{La}$ & $\mathrm{Sn}$ & $\mathrm{Pb}$ & & & $\mathrm{v}$ & $\mathrm{Nb}$ & $\mathrm{Cr}$ & Mo & Co & $\mathrm{Ni}$ \\
\hline $38-1,62-65$ & Sap. basalt, aphyric & (B) & 6.7 & 0.0 & 260.8 & 0.0 & 0.0 & 108.8 & 139.2 & 0.0 & 17.5 & 23.5 & 0.0 & 4.3 & 0.0 & 130 & 0.0 & 379.0 & 13.6 & 100.5 & 0.0 & 114.0 & 112.8 \\
\hline \multirow[t]{2}{*}{$38-2,56-69$} & Partly sap. basalt, aphyric & (M) & 6.2 & 3.2 & 99.0 & 1.5 & 1.3 & 89.3 & 59.0 & 0.0 & 16.1 & 25.2 & 14.4 & 0.0 & 0.0 & 90 & 8.0 & 483.6 & 40.0 & 95.4 & 0.0 & 140.0 & 93.0 \\
\hline & & & 6.4 & 3.1 & 109.6 & 0.4 & 0.7 & 90.6 & 60.2 & 7.8 & 16.2 & 31.1 & 11.7 & 2.4 & 0.0 & 110 & 4.0 & 426.7 & 25.8 & 93.5 & 3.7 & 152.2 & 100.9 \\
\hline $39-1,2-4$ & Partly sap. basalt, f-mphyric & (T) & 5.3 & 6.4 & 154.5 & 0.0 & 0.2 & 91.3 & 107.5 & 0.0 & 13.9 & 21.4 & 0.0 & 4.4 & 0.0 & 100 & 0.0 & 299.7 & 14.6 & 86.0 & 0.0 & 107.0 & 116.9 \\
\hline $40-1,9-12$ & Partly sap. basalt, aphyric & (T) & 3.3 & 13.4 & 138.4 & 0.0 & 0.1 & 93.3 & 50.2 & 3.1 & 14.3 & 33.6 & 0.0 & 5.1 & 0.0 & 140 & 0.1 & 266.6 & 17.4 & 78.3 & 0.0 & 70.7 & 79.0 \\
\hline \multirow[t]{2}{*}{$40-1,79-82$} & Slightly sap. basalt (?olivine & (M) & 8.5 & 0.7 & 179.4 & 0.2 & 0.8 & 85.0 & 102.5 & 2.1 & 16.2 & 40.0 & 6.5 & 4.6 & 4.2 & 170 & 4.5 & 370.0 & 30.8 & 26.0 & 1.0 & 117.2 & 73.1 \\
\hline & f-cpx-mphyric & & 8.2 & 0.7 & 173.7 & 0.2 & 0.7 & 83.6 & 89.1 & 0.0 & 16.9 & 42.6 & 3.0 & 4.6 & 0.1 & 180 & 2.9 & 350.5 & 31.6 & 25.4 & 1.3 & 104.3 & 69.7 \\
\hline $40-4,80-83$ & $\begin{array}{l}\text { Partly sap. basalt, f-cpx- } \\
\text { mphyric }\end{array}$ & (B) & 7.2 & 4.7 & 132.4 & 0.0 & 0.2 & 81.1 & 109.1 & 1.8 & 14.4 & 40.5 & 0.0 & 6.7 & 0.0 & 180 & 0.0 & 239.5 & 20.0 & 21.0 & 0.0 & 81.9 & 81.2 \\
\hline $42-2,42-46$ & Partly sap. basalt, $f$-mphyric & (?) & 5.2 & 0.0 & 193.8 & 0.0 & 0.0 & 87.2 & 84.3 & 0.8 & 15.0 & 31.6 & 0.0 & 3.2 & 0.0 & 110 & 0.8 & 240.4 & 16.9 & 117.8 & 1.2 & 77.0 & 113.1 \\
\hline $43-1,20-23$ & Sap. basalt, $f$-mphyric & (T) & 4.1 & 15.8 & 125.8 & 0.0 & 0.5 & 86.4 & 61.4 & 17.0 & 13.2 & 30.8 & 0.0 & 6.9 & 0.0 & 140 & 5.4 & 282.7 & 16.7 & 39.3 & 0.0 & 85.6 & 76.6 \\
\hline $43-1,62-66$ & Basaltic tuff (?) & & 6.5 & 14.5 & 282.1 & 0.1 & 0.6 & 73.1 & 113.0 & 0.0 & 10.7 & 12.2 & 0.0 & 5.0 & 0.0 & 120 & 1.5 & 309.0 & 17.0 & 33.7 & 0.0 & 86.9 & 77.4 \\
\hline $43-2,111-114$ & Basalt, f-mphyric & (?) & 6.4 & 0.5 & 181.5 & 0.0 & 0.1 & 72.4 & 55.3 & 8.3 & 9.6 & 29.9 & 0.0 & 7.9 & 0.0 & 130 & 3.7 & 253.5 & 16.0 & 37.4 & 1.8 & 126.7 & 114.1 \\
\hline $44-2,48-51$ & Partly sap. basalt f-mphyric & (?) & 7.2 & 0.3 & 67.2 & 0.0 & 0.4 & 81.3 & 85.0 & 2.2 & 14.8 & 46.8 & 0.0 & 5.3 & 0.0 & 170 & 2.8 & 359.1 & 26.0 & 53.1 & 0.9 & 91.3 & 97.0 \\
\hline $45-1,44-47$ & Vitroclastic tuff & & 16.9 & 17.6 & 96.6 & 0.0 & 1.0 & 72.8 & 61.6 & 60.0 & 10.5 & 28.1 & 5.7 & 11.6 & 00 & 110 & 2.8 & 247.0 & 9.0 & 23.5 & & 62.6 & 71.8 \\
\hline & & & 16.6 & 16.6 & 95.4 & 0.2 & 0.8 & 73.4 & 65.7 & 59.9 & 11.0 & 33.2 & 8.3 & 11.0 & 0.0 & 170 & 4.5 & 260.0 & 14.8 & 23.0 & 7.3 & 51.9 & 62.1 \\
\hline $45-1,87-91$ & Vitroclastic tuff & & 18.1 & 12.2 & 289.2 & 0.0 & 0.5 & 78.2 & 73.3 & 53.3 & 9.9 & 59.4 & 2.9 & 7.0 & 0.0 & 130 & 1.6 & 164.6 & 9.3 & 20.3 & 1.4 & 72.4 & 86.9 \\
\hline $45-2,114-118$ & Sap. ?tuff & & 6.9 & 16.3 & 221.8 & 0.0 & 0.0 & 73.3 & 59.0 & 1.1 & 9.9 & 18.4 & 0.0 & 7.1 & 0.0 & 90 & 0.0 & 194.5 & 10.0 & 35.7 & 0.0 & 101.2 & 131.2 \\
\hline $45-3,79-83$ & Sap. ?tuff & (?M) & 5.0 & 3.6 & 184.0 & 0.0 & 0.1 & 88.4 & 92.8 & 0.5 & 13.2 & 25.8 & 0.0 & 5.4 & 0.0 & 90 & 0.5 & 295.6 & 16.6 & 59.0 & 0.0 & 83.1 & 120.9 \\
\hline $45-4,16-20$ & Basaltic breccia/tuff & & 3.5 & 0.0 & 164.8 & 0.0 & 0.4 & 68.0 & 60.5 & 22.3 & 10.5 & 21.4 & 0.0 & 9.6 & 0.0 & 70 & 1.9 & 163.1 & 13.3 & 37.5 & 2.9 & 130.8 & 158.1 \\
\hline $45-5,17-19$ & Basalt, (cpx-rich), f-mphyric & (?) & 4.9 & 0.1 & 124.8 & 0.0 & 0.5 & 78.6 & 84.8 & 10.1 & 14.7 & 24.2 & 0.0 & 5.6 & 4.8 & 80 & 2.3 & 318.8 & 26.8 & 49.8 & 3.8 & 106.9 & 121.0 \\
\hline & & & 5.2 & 0.2 & 112.4 & 0.6 & 0.9 & 82.4 & 62.2 & 6.0 & 14.4 & 23.8 & 6.7 & 5.3 & 2.0 & 90 & 5.9 & 379.1 & 30.1 & 51.4 & 0.9 & 526.6 & 119.8 \\
\hline $46-1,121-125$ & Basalt, (cpx-rich), f-mphyric & (?) & 5.0 & 0.0 & 284.6 & 0.0 & 0.4 & 79.8 & 91.5 & 9.7 & 14.1 & 28.5 & 0.0 & 6.2 & 4.3 & 100 & 1.3 & 316.4 & 30.2 & 49.0 & 4.1 & 127.9 & 128.7 \\
\hline & & (?) & 5.8 & 0.0 & 315.2 & 0.0 & 0.4 & 76.1 & 103.9 & 14.5 & 14.4 & 30.2 & 0.4 & 7.0 & 19.1 & 100 & 3.8 & 275.2 & 25.9 & 49.3 & 2.8 & 111.0 & 118.1 \\
\hline $46-3,56-59$ & Basalt, (cpx-rich), f-mphyric & (?) & 5.3 & 0.0 & 140.8 & 0.0 & 0.4 & 77.8 & 76.9 & 16.9 & 14.2 & 31.9 & 0.8 & 5.8 & 4.5 & 110 & 1.8 & 340.0 & 40.2 & 49.6 & 7.9 & 116.7 & 127.9 \\
\hline & & & 5.4 & 0.0 & 147.9 & 0.0 & 0.4 & 77.9 & 68.7 & 14.8 & 14.1 & 36.5 & 2.2 & 6.8 & 19.6 & 130 & 4.8 & 273.3 & 32.8 & 44.9 & 3.8 & 103.1 & 112.0 \\
\hline $46-4,53-56$ & Basalt, mcryst., aphyric & (B) & 5.8 & 0.8 & 164.1 & 0.2 & 0.7 & 79.7 & 78.3 & 2.5 & 13.7 & 23.5 & 7.0 & 6.2 & 8.1 & 90 & 4.3 & 255.7 & 26.2 & 46.4 & 1.4 & 94.8 & 104.9 \\
\hline & & & 6.2 & 0.7 & 163.5 & 0.6 & 0.8 & 81.8 & 82.3 & 2.1 & 14.7 & 25.3 & 11.8 & 4.0 & 0.0 & 80 & 3.7 & 369.2 & 36.7 & 52.2 & 1.9 & 94.6 & 107.4 \\
\hline $2-124$ & Sap. & (T) & 6.2 & 1.7 & 307.5 & 0.0 & 0.3 & 93.5 & 58.1 & 2.9 & 12.9 & 43.3 & 0.0 & 7.4 & 0.0 & 110 & 1.1 & 342.7 & 17.8 & 49.2 & 1.4 & 98.2 & 111.0 \\
\hline $46-6,33-36$ & It $f$-mphyric & (?M) & 4.0 & 0.5 & 78.3 & 0.0 & 0.2 & 80.1 & 75.4 & 2.3 & 14.0 & 49.6 & 0.0 & 5.3 & 0.0 & 160 & 1.9 & 295.4 & 17.4 & 46.8 & 0.2 & 92.8 & 98.4 \\
\hline $47-3,73-76$ & Basalt f-mphyric & (?M) & 5.8 & 0.2 & 85.6 & 0.6 & 0.9 & 86. & 89.9 & 0.0 & 15 & 37.3 & 12.2 & 3.6 & 3. & 13 & 7.9 & 423.1 & 36.8 & 46.4 & 1.4 & 105.2 & 86.7 \\
\hline & & & 5.2 & 0.3 & 86.7 & 0.1 & 0.7 & 83.9 & 65.8 & 13.8 & 15.2 & 40.5 & 6.8 & 6.4 & 6.6 & 130 & 4.8 & 367.9 & 31.0 & 48.5 & 5.9 & 129.4 & 111.3 \\
\hline $47-4,181-184$ & Sap. b & (B) & 5.8 & 2.0 & 232.7 & 0.1 & 0.4 & 90. & 90 & 0.0 & 15.5 & 30.7 & 7. & 4. & 0.4 & 130 & 7 & 385.8 & 21.8 & 48.6 & 0.0 & 89.8 & 97.2 \\
\hline $47-4,128-13$ & Sap. b & (T) & 4.3 & 10.3 & 97.4 & 0.0 & 0. & 71 & 54 & 5.2 & 9.3 & 9. & 0. & 5. & 0. & 90 & 3. & 298.7 & 13.5 & 60.9 & 0.0 & 106.4 & 120.1 \\
\hline $48-1,13-16$ & Partly sap. basalt $f$-mphyric & (M) & 3.8 & 0.0 & 94.0 & 0.0 & 0.3 & 84.9 & 84.6 & 0.0 & 14.0 & 34.9 & 0.0 & 5.3 & 0.0 & 110 & 0.2 & 305.4 & 15.4 & 93.9 & 0.0 & 106.7 & 143.4 \\
\hline $48-2,60-65$ & Basalt f-mphyric & (?M) & 6.0 & 0.0 & 242.8 & 0.0 & 0.5 & 83.3 & 105.8 & 11.7 & 16.7 & 48.3 & 4.7 & 6.2 & 10.5 & 180 & 2.9 & 292.4 & 21.4 & 84.7 & 4.4 & 108.6 & 121.3 \\
\hline & & & 6.1 & 0. & 211.2 & 0.0 & 0.5 & 80. & 81.3 & 11.3 & 14.9 & 33.0 & 2. & 6.2 & 5. & 13 & 2.8 & 346.2 & 28.4 & 86.9 & 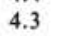 & 109.8 & 134.4 \\
\hline 48-3 & $\mathrm{Sl}$ & (? & 6.5 & $0.1-10$ & 151.3 & 0.2 & 0.5 & 79.4 & 72 & 6.9 & 13. & 46.5 & 10.2 & 4.6 & 0.0 & 170 & 6. & 390.5 & 37.7 & 112.0 & 3.2 & 100.2 & 124.2 \\
\hline $48-6,53-58$ & Slightly sap. basalt, $\mathrm{f}$-mphyric & (?M) & 5.0 & 0.0 & 178.7 & 0.6 & 0.2 & 82.0 & 72.1 & 1.3 & 14.7 & 38.5 & 0.0 & 4.9 & 0.0 & 130 & 0.9 & 299.9 & 18.7 & 102.1 & 0.0 & 86.6 & 112.0 \\
\hline
\end{tabular}




\begin{tabular}{|c|c|c|c|c|c|c|c|c|c|c|c|c|c|c|c|c|c|c|c|c|c|c|c|}
\hline $48-6,74-76$ & Partly sap. basalt, $f$-mphyric & (?M) & 5.4 & 0.4 & 534.7 & 0.0 & 0.4 & 92.2 & 98.5 & 0.0 & 15.4 & 26.8 & 0.0 & 4.5 & 0.0 & 100 & 1.7 & 377.0 & 21.3 & 110.0 & 0.0 & 89.4 & 126.6 \\
\hline $48-6,85-87$ & Basaltic tuff & (?M) & 4.9 & 5.3 & 130.0 & 0.0 & 0.0 & 94.4 & 160.2 & 0.0 & 15.0 & 44.9 & 0.0 & 3.0 & 0.0 & 110 & 0.0 & 250.6 & 3.4 & 83.0 & 0.0 & 91.0 & 145.0 \\
\hline $48-6,122-125$ & Slightly sap. basalt, f-mphyric & (?M) & 4.8 & 0.0 & 318.7 & 0.0 & 0.1 & 81.4 & 80.0 & 2.5 & 14.1 & 36.3 & 0.0 & 5.7 & 0.0 & 120 & 2.3 & 309.4 & 23.1 & 100.2 & 0.0 & 90.3 & 115.8 \\
\hline $50-1,67-71$ & Sap. basalt, aphyric & (T) & 3.0 & 0.1 & 21.8 & 0.5 & 0.1 & 80.1 & 65.6 & 0.0 & 12.1 & 34.7 & 0.0 & 6.5 & 0.5 & 80 & 6.9 & 252.3 & 18.6 & 104.5 & 0.0 & 85.5 & 125.4 \\
\hline $50-2,20-27$ & Partly sap. basalt, aphyric & (M) & 4.5 & 0.0 & 115.5 & 0.0 & 0.2 & 75.2 & 78.1 & 0.2 & 9.9 & 24.0 & 0.0 & 5.3 & 0.0 & 70 & 1.1 & 278.8 & 13.8 & 110.1 & 0.0 & 112.9 & 152.9 \\
\hline $50-3,32-35$ & Slightly sap. basalt, aphyric & (B) & 5.0 & 0.0 & 101.5 & 0.0 & 0.1 & 78.2 & 53.4 & 0.0 & 11.4 & 25.2 & 0.0 & 1.8 & 0.0 & 80 & 0.9 & 249.3 & 10.1 & 110.1 & 0.0 & 89.6 & 147.6 \\
\hline $50-3,71-75$ & Sap. basalt, aphyric & (T) & 3.6 & 24.5 & 497.0 & 0.0 & 0.2 & 77.2 & 59.6 & 14.2 & 8.4 & 6.4 & 0.0 & 7.3 & 0.0 & 80 & 0.0 & 212.5 & 9.5 & 63.4 & 1.4 & 96.9 & 128.2 \\
\hline \multirow[t]{3}{*}{$51-1,54-57$} & Basalt, f-phyric & (M) & 6.1 & 0.0 & 270.4 & 0.0 & 0.2 & 74.1 & 79.3 & 3.3 & 13.2 & 24.9 & 0.0 & 5.4 & 0.0 & 80 & 1.4 & 263.7 & 16.9 & 91.6 & 0.0 & 97.3 & 139.0 \\
\hline & Basalt, f-phyric & & 3.0 & 1.2 & 324.6 & 0.0 & 0.3 & 77.2 & 105.3 & 7.5 & 13.3 & 23.1 & 0.0 & 5.5 & 6.8 & 80 & 1.4 & 264.2 & 17.7 & 84.2 & 2.4 & 100.9 & 136.3 \\
\hline & & & 3.4 & 1.3 & 340.4 & 0.3 & 0.6 & 79.7 & 91.5 & 11.0 & 13.6 & 28.7 & 8.0 & 4.7 & 4.5 & 100 & 4.5 & 252.4 & 17.1 & 95.6 & 4.6 & 92.3 & 122.1 \\
\hline \multirow{2}{*}{$51-2,77-80$} & Basalt, f-phyric & (M) & 5.0 & 0.0 & 199.9 & 0.0 & 0.4 & 76.0 & 94.6 & 0.0 & 14.0 & 27.2 & 0.0 & 4.4 & 6.0 & 90 & 2.0 & 340.7 & 20.5 & 93.7 & 0.0 & 111.4 & 125.2 \\
\hline & & & 5.2 & 0.0 & 213.5 & 0.0 & 0.3 & 78.0 & 80.3 & 5.1 & 13.5 & 26.3 & 0.7 & 6.0 & 9.9 & 90 & 4.0 & 364.7 & 22.5 & 97.9 & 1.1 & 113.2 & 131.8 \\
\hline $52-3,8-11$ & Basaltic sap. ?flow-breccia & (T) & 2.2 & 5.8 & 138.0 & 0.0 & 0.4 & 82.6 & 62.4 & 8.2 & 9.5 & 19.7 & 0.0 & 7.2 & 0.0 & 100 & 0.0 & 189.0 & 9.9 & 54.1 & 1.8 & 124.0 & 154.6 \\
\hline $52-3,140-143$ & Sap. basalt, meryst. & (T) & 1.5 & 7.3 & 145.5 & 0.0 & 0.3 & 74.0 & 69.0 & 11.1 & 8.8 & 41.1 & 0.0 & 6.8 & 0.0 & 140 & 0.2 & 178.7 & 11.8 & 43.3 & 6.2 & 145.9 & 172.0 \\
\hline \multirow[t]{2}{*}{$53-1,77-80$} & Slightly sap. basalt, f-mphyric & (?M) & 5.6 & 0.0 & 236.8 & 0.0 & 0.6 & 88.0 & 74.6 & 2.8 & 15.7 & 30.8 & 8.2 & 0.1 & 0.0 & 120 & 0.5 & 380.6 & 23.6 & 90.1 & 1.4 & 124.2 & 123.0 \\
\hline & & & 5.0 & 0.0 & 224.5 & 0.0 & 0.3 & 83.5 & 79.4 & 2.1 & 13.4 & 33.1 & 0.9 & 3.0 & 0.0 & 110 & 0.7 & 272.8 & 12.5 & 81.6 & 2.2 & 111.4 & 120.0 \\
\hline $53-2,85-88$ & Sap. basalt, aphyric & (B) & 4.0 & 0.0 & 468.2 & 0.0 & 0.1 & 90.5 & 95.8 & 0.0 & 12.9 & 25.0 & 0.0 & 3.1 & 0.0 & 70 & 0.0 & 313.0 & 14.6 & 79.8 & 0.0 & 102.7 & 137.8 \\
\hline $53-4,31-34$ & salt, $f$-mphyric & (?) & 4.4 & 3.0 & 440.6 & 0.0 & 0.0 & 86.8 & 86.8 & 0.5 & 13.4 & 26.4 & 0.0 & 4.3 & 0.0 & 90 & 0.1 & 211.3 & 5.9 & 23.7 & 0.0 & 108.5 & 94.6 \\
\hline $53-4,76-79$ & Partly sap. basalt, f-mphyric & (T) & 6.6 & 0.3 & 200.2 & 0.0 & 0.4 & 86.2 & 73. & 2.0 & 15. & 40.6 & 4.3 & 3.5 & 0.0 & 170 & 2.8 & 381.2 & 23.8 & 47.5 & 1.5 & 102.5 & 93.9 \\
\hline $54-1,64-67$ & Sap. basalt, f-mphyric & (M) & 6.9 & 7.4 & 243.2 & 0.0 & 0.3 & 88.8 & 77.3 & 0.0 & 14.7 & 28.6 & 6.3 & 6.2 & 5.1 & 160 & 8.0 & 268.4 & 10.9 & 22.9 & 0.0 & 88.5 & 77.0 \\
\hline $54-1,89-92$ & Sap. basalt, f-mphyric & (M) & 4.2 & 0.0 & 270.1 & 0.0 & 0.2 & 85.4 & 132.3 & 0.0 & 16.3 & 26.5 & 3.1 & 3.5 & 0.3 & 110 & 0.1 & 322.4 & 13.5 & 39.3 & 0.0 & 109.9 & 98.3 \\
\hline \multirow{2}{*}{$54-5,81-85$} & Slightly sap. basalt, aphyric & (M) & 4.7 & 0.0 & 158.8 & 1.0 & 0.5 & 77.8 & 83.2 & 3.4 & 14.1 & 28.7 & 0.0 & 5.5 & 9.5 & 90 & 5.9 & 347.7 & 17.2 & 25.7 & 1.9 & 109.6 & 88.0 \\
\hline & & & 5.3 & 0.0 & 172.7 & 0.9 & 0.7 & 79.7 & 80.9 & 1.1 & 15.8 & 35.5 & 11.1 & 3.2 & 4.9 & 110 & 8.1 & 451.7 & 33.5 & 26.2 & 1.5 & 91.4 & 75.0 \\
\hline $54-5,83-86$ & alt, f-mphy & (M) & 7.5 & 0.2 & 140.4 & 0.2 & 0.4 & 86.2 & 83.7 & 0.0 & 16.6 & 29.8 & 8.8 & 5.2 & 0.0 & 100 & 7.3 & 342.1 & 19.0 & 28.1 & 0.0 & 82.5 & 85.7 \\
\hline $55-1,36-38$ & Sap. & (T) & 3.3 & 7.7 & 198.9 & 0.4 & 0.7 & 91.7 & 47. & 0.3 & 12.5 & 100.2 & 17.2 & 4.9 & 0. & 90 & 5.4 & 322.0 & 23 & 99.6 & 0.0 & 89.3 & 87.3 \\
\hline $55-3,71-74$ & Sap. basalt (?agglomeratic) & (B) & 3.4 & 4.7 & 162.7 & 0.4 & 0.9 & 70.7 & 75.9 & 0.0 & 10.3 & 13.7 & 9.4 & 6.5 & 0.0 & 70 & 4.9 & 252.5 & 19.3 & 67.4 & 0.0 & 74.2 & 92.8 \\
\hline $55-3,113-116$ & Partly sap. basalt, f-phyric & (T) & 4.8 & 0.0 & 83.2 & 0.0 & 0.1 & 90.4 & 95.8 & 3.0 & 15.2 & 28.5 & 0.0 & 7.3 & 4.4 & 120 & 1.4 & 266.6 & 15.7 & 62.7 & 0.0 & 106.0 & 142.1 \\
\hline \multirow[t]{2}{*}{$55-5,92-95$} & Partly sap. basalt, f-mphyric & (M) & 5.7 & 0.0 & 345.0 & 0.8 & 0.9 & 75.9 & 85. & 4.0 & 14.0 & 27.6 & 10.0 & 2. & 6. & 90 & 8. & 392.0 & 33 & 75.7 & 3.6 & 93.8 & 95.2 \\
\hline & & & 5.1 & 0.0 & 355.6 & 1.1 & 0.8 & 84.4 & 49.7 & 0.0 & 14.0 & 29.5 & 6.0 & 0.0 & 0.0 & 90 & 4.7 & 421.4 & 37.9 & 82.0 & 1.8 & 92.6 & 107.0 \\
\hline \multirow[t]{2}{*}{$55-6,44-47$} & Slightly sap. basalt, f-phyric & (?) & 5.6 & 0.3 & 127.4 & 0.0 & 0.4 & 85.1 & 93.7 & 5.6 & 16.3 & 31.0 & 2.6 & 5.3 & 9.0 & 140 & 1.9 & 276.5 & 13.5 & 75.5 & 0.0 & 105.6 & 134.0 \\
\hline & & & 5.7 & 0.2 & 113.3 & 0.1 & 0.8 & 5.8 & 68.5 & 4.2 & 14.6 & 32.1 & 14.3 & 2.8 & 3.6 & 120 & 4.1 & 404.0 & 25 & 83.6 & 1.3 & 100.1 & 118.3 \\
\hline \multirow[t]{2}{*}{$56-1,3-6$} & Slightly sap. basalt, f-phyric & (B) & 6.7 & 0.4 & 240.4 & 0.9 & 0.6 & 89.4 & 88.0 & 0.0 & 16.8 & 27.4 & 11.2 & 2.1 & 12.6 & 110 & 6.5 & 454.3 & 29 & 81 & 0.0 & 104.6 & 117.2 \\
\hline & & & 6.2 & 0.2 & 250.6 & 0.0 & 0.4 & 84.3 & 82.9 & 7.5 & 15.3 & 37.0 & 0.0 & 5.5 & 13.9 & 130 & 3.2 & 252.4 & 17 & 70.0 & 2.4 & 92.2 & 112.5 \\
\hline $56-2,81-84$ & $\begin{array}{l}\text { Slightly sap, basalt, sparsely } \\
\text { mphyric }\end{array}$ & (M) & 5.9 & 0.0 & 141.4 & 0.1 & 0.6 & 83.7 & 61.8 & 0.0 & 13.5 & 22.8 & 6.7 & 2.6 & 0.0 & 100 & 0.5 & 404.4 & 24.2 & 90.5 & 0.0 & 111.4 & 120.7 \\
\hline $56-3,91-94$ & $\begin{array}{l}\text { Slightly sap. basalt, sparsely } \\
\text { mphyric }\end{array}$ & (B) & 5.3 & 0.0 & 676.5 & 0.0 & 0.1 & 83.6 & 87.5 & 0.0 & 13.1 & 27.1 & 0.0 & 2.0 & 0.0 & 100 & 0.0 & 276.0 & 6.3 & 79.6 & 0.0 & 98.5 & 142.1 \\
\hline $57-2$ & basalt, $f, c p x$-phyr & (M) & 5.7 & 0. & 247.1 & . & 0. & 85 & 94 & 0. & 10 & 28 & . & 3.4 & 0. & 9 & 0.0 & 356 & 16 & 52.7 & 0.0 & 97.2 & 143.4 \\
\hline $58-1,89-91$ & Partly & (M) & 4.1 & 2. & 58.0 & 0. & 0.1 & 82 & 58 & 2.2 & 13 & 26. & 0. & 2. & 0 & 9 & 0. & 26 & 15 & 257.7 & 0.5 & 92.2 & 167.1 \\
\hline $58-2,117-120$ & cpx-phyric & (T) & 4.1 & 0.0 & 198.6 & 0.0 & 0.1 & 84. & 86 & 0.0 & 13 & 46 & 0. & 3. & 0. & 90 & 0.0 & 270.2 & 17.1 & 241.4 & 0.0 & 96.1 & 164.7 \\
\hline $59-1,36-38$ & $\begin{array}{l}\text { Partly sap. basalt, f., cpx- } \\
\text { phyric }\end{array}$ & (?B) & 4.5 & 0.0 & 419.5 & 0.0 & 0.4 & 82.5 & 72.2 & 0.0 & 13.1 & 19.1 & 0.0 & 3.8 & 0.0 & 70 & 0.9 & 305.7 & 17.6 & 245.1 & 0.0 & 98.0 & 166.0 \\
\hline \multirow[t]{2}{*}{$59-2,115-118$} & Partly sap. basalt, f-mphyric & (T) & 6. & 0. & 16 & 0. & 0. & 89 & 71 & 6. & 14 & 27 & 1 & 3. & 0 & 13 & 3. & & 16 & 23 & 4.3 & 78.1 & 136.5 \\
\hline & & & 6.9 & 0.1 & 157.3 & 0.1 & 0.4 & 88.1 & 69. & 10.5 & 14.5 & 32.2 & 3. & 2. & 2.7 & 130 & 2.2 & 226.3 & 11. & 246.6 & 6.7 & 70.7 & 132.0 \\
\hline $59-4,93-95$ & Partly sap. basalt, f-phyric & (?M) & 9.3 & 0.0 & 95.4 & 0.0 & 0.3 & 69.9 & 52.0 & 6.5 & 11.2 & 21.0 & 5.9 & 4.6 & 0.0 & 70 & 1.9 & 284.5 & 24.3 & 252.3 & 1.4 & 97.0 & 175.2 \\
\hline
\end{tabular}


Table 17. Trace elements of basalts from Hole 554A.

\begin{tabular}{|c|c|c|c|c|c|c|c|c|c|c|c|c|c|c|c|c|c|c|c|c|c|c|c|}
\hline \multirow{2}{*}{$\begin{array}{c}\text { Core-Section } \\
\text { (interval in cm) }\end{array}$} & \multirow[b]{2}{*}{ Brief lithology } & \multirow{2}{*}{$\begin{array}{l}\text { Flow } \\
\text { position }\end{array}$} & \multicolumn{2}{|c|}{ IA } & \multicolumn{2}{|c|}{ IB } & \multicolumn{2}{|c|}{ IIA } & \multirow{2}{*}{$\frac{\text { IIB }}{\mathrm{Zn}}$} & \multicolumn{2}{|c|}{ IIIA } & \multicolumn{2}{|c|}{ IIIB } & \multicolumn{2}{|c|}{ IIIA } & \multirow{2}{*}{$\frac{\mathrm{IVB}}{\mathrm{Zr}}$} & \multirow{2}{*}{$\frac{\mathrm{VA}}{\mathrm{Bi}}$} & \multicolumn{2}{|c|}{ VB } & \multicolumn{2}{|c|}{ VIB } & \multicolumn{2}{|c|}{ VIII } \\
\hline & & & $\mathbf{L i}$ & $\mathbf{R b}$ & $\mathrm{Cu}$ & $\mathrm{Ag}$ & $\mathrm{Be}$ & $\mathrm{Sr}$ & & B & $\mathrm{Ga}$ & $\mathbf{Y}$ & $\mathrm{La}$ & $\mathrm{Sn}$ & $\mathrm{Pb}$ & & & $\mathrm{v}$ & $\mathrm{Nb}$ & $\mathrm{Cr}$ & Mo & Co & $\mathrm{Ni}$ \\
\hline \multirow{2}{*}{$7-1,63-66$} & Basalt, f-, cpx-microphyric & (?T) & 6.9 & 8.6 & 136.9 & 0 & 0.1 & 90.2 & 81.5 & 31.6 & 14.2 & 32.6 & 0.0 & 6.0 & 10.1 & 120 & 0.9 & 249.2 & 18.1 & 35.1 & 1.7 & 189.1 & 107.1 \\
\hline & & & 8.6 & 11.1 & 139.2 & 1.1 & 0.7 & 97.5 & 60.0 & 21.0 & 16.8 & 23.6 & 12.5 & 1.4 & 0.0 & 100 & 3.7 & 372.6 & 47.9 & 42.4 & 0.0 & 151.2 & 86.7 \\
\hline \multirow[t]{2}{*}{$7-3,10-12$} & Basalt, f-, cpx-microphyric & (?M) & 9.9 & 3.0 & 139.8 & 0.2 & 0.3 & 92.0 & 88.6 & 2.8 & 15.3 & 28.0 & 0.0 & 5.1 & 13.0 & 110 & 3.1 & 274.9 & 28.9 & 35.8 & 0.0 & 103.9 & 84.0 \\
\hline & & & 9.8 & 2.8 & 147.1 & 0.0 & 0.5 & 92.3 & 79.6 & 4.1 & 15.0 & 26.5 & 0.0 & 4.7 & 8.3 & 100 & 1.2 & 368.0 & 36.0 & 42.7 & 0.0 & 120.3 & 95.2 \\
\hline \multirow[t]{2}{*}{$7-4,25-28$} & Basalt, glassy, carbonated, & (B) & 8.9 & 5.0 & 160.1 & 0.0 & 0.2 & 87.8 & 75.2 & 8.7 & 15.1 & 29.4 & 0.0 & 6.4 & 11.7 & 120 & 1.7 & 217.3 & 21.9 & 34.0 & 0.8 & 124.9 & 89.4 \\
\hline & 18 & & 9.2 & 5.0 & 163.3 & 0. & 0.2 & 91.6 & 69.4 & 14.8 & 15.1 & 31.9 & 0.0 & 5.3 & 10.4 & 120 & 3.3 & 269.0 & 35.7 & 36.4 & 3.7 & 112.8 & 80.5 \\
\hline $8-1,99-102$ & Basalt, ?olivine-, carbonated, & (T) & 6.9 & 1.5 & 113.8 & 0.0 & 0.1 & 93.7 & 109.9 & 30.1 & 14.4 & 25.8 & 0.0 & 8.8 & 12.7 & 110 & 0.8 & 329.4 & 32.3 & 168.1 & 0.0 & 127.5 & 142.1 \\
\hline $9-1,75-78$ & $\begin{array}{l}\text { Basalt, (ex-conglomerate) f- } \\
\text { microphyric }\end{array}$ & & 13.7 & 8.5 & 156.5 & 0.1 & 0.5 & 99.9 & 101.6 & 34.5 & 17.1 & 33.9 & 4.4 & 3.9 & 0.0 & 130 & 2.3 & 333.8 & 28.1 & 208.1 & 1.6 & 85.6 & 94.3 \\
\hline \multirow[t]{2}{*}{$14-1,36-39$} & $\begin{array}{l}\text { Basalt, (ex-conglomerate), f- } \\
\text { microphyric }\end{array}$ & (?) & 31.1 & 7.1 & 170.6 & 0.7 & 0.5 & 88.4 & 70.4 & 0.0 & 15.2 & 24.3 & 7.2 & 3.4 & 0.0 & 80 & 2.6 & 387.8 & 37.3 & 224.0 & 0.0 & 87.8 & 136.8 \\
\hline & & Mean & 11.7 & 5.8 & 147.5 & 0.2 & 0.3 & 92.6 & 81.8 & 16.4 & 15.3 & 28.4 & 2.7 & 5.0 & 7.4 & 110 & 2.2 & 311.3 & 31.8 & 91.8 & 0.9 & 122.5 & 101.8 \\
\hline
\end{tabular}




\begin{tabular}{|c|c|c|c|c|c|c|c|c|c|c|c|c|c|c|c|c|c|c|c|c|c|c|c|}
\hline \multirow{2}{*}{$\begin{array}{c}\text { Core-Section } \\
\text { (interval in cm) }\end{array}$} & \multirow[b]{2}{*}{ Brief lithology } & \multirow{2}{*}{$\begin{array}{l}\text { Flow } \\
\text { position }\end{array}$} & \multicolumn{2}{|c|}{ IA } & \multicolumn{2}{|c|}{ IB } & \multicolumn{2}{|c|}{ IIA } & \multirow{2}{*}{$\frac{\mathrm{IIB}}{\mathrm{Zn}}$} & \multicolumn{2}{|c|}{ IIIA } & \multicolumn{2}{|c|}{ IIIB } & \multicolumn{2}{|c|}{ IVA } & \multirow{2}{*}{$\frac{\text { IVB }}{\mathrm{Zr}_{\mathrm{r}}}$} & \multirow{2}{*}{$\frac{\mathrm{VA}}{\mathrm{Bi}}$} & \multicolumn{2}{|c|}{ vB } & \multicolumn{2}{|c|}{ VIB } & \multicolumn{2}{|c|}{ VIII } \\
\hline & & & $\mathrm{Li}$ & $\mathbf{R b}$ & $\mathrm{Cu}$ & $\mathrm{Ag}$ & $\mathrm{Be}$ & Sr & & B & $\mathrm{Ga}$ & $\mathbf{Y}$ & $\mathrm{La}$ & Sn & $\mathrm{Pb}$ & & & $\mathrm{v}$ & $\mathrm{Nb}$ & $\mathrm{Cr}$ & Mo & Co & $\mathrm{Ni}$ \\
\hline $68-2,85-88$ & $\begin{array}{l}\text { Partly sap. basalt; f-, cpx, } \\
\text { cumulophyric }\end{array}$ & (?) & 8.8 & 0.0 & 327.1 & 0.0 & 0.4 & 143.0 & 130.1 & 0.0 & 18.3 & 26.5 & 0.0 & 2.9 & 0.0 & 130 & 0.0 & 383.8 & 22.0 & 167.0 & 0.0 & 77.7 & 125 \\
\hline $68-3,122-126$ & $\begin{array}{l}\text { Partly sap. basalt; } f-, c p x, \\
\text { cumulophyric }\end{array}$ & (?) & 6.5 & 0.0 & 293.5 & 0.0 & 0.3 & 116.5 & 77.5 & 0.0 & 17.8 & 36.4 & 0.0 & 6.9 & 2.3 & 160 & 2.2 & 338.4 & 20.2 & 135.5 & 0.0 & 91.0 & 142 \\
\hline $69-2,37-40$ & $\begin{array}{l}\text { Partly sap. basalt; f-, cpx, } \\
\text { cumulophyric }\end{array}$ & (?) & 6.0 & 0.0 & 287.5 & 0.0 & 0.3 & 107.1 & 113.9 & 0.0 & 16.3 & 36.3 & 0.0 & 6.4 & 5.9 & 140 & 0.0 & 288.6 & 19.6 & 122.5 & 0.0 & 82.7 & 140 \\
\hline $69-3,113-116$ & $\begin{array}{l}\text { Partly sap. basalt; f-, cpx, } \\
\text { cumulophyric (?olivine-) }\end{array}$ & (?) & $\begin{array}{l}6.4 \\
6.5\end{array}$ & 0.5 & 94.7 & 0.0 & 0.3 & 123.4 & 120.8 & 3.6 & 17.9 & 35.5 & 0.0 & 4.7 & 8.1 & 130 & 2.7 & 351.4 & 18.8 & 141.8 & 0.9 & 83.2 & 12 \\
\hline $70-1,135-138$ & Sap. basalt; aphyric & (B) & $\begin{array}{l}6.5 \\
7.1\end{array}$ & $\begin{array}{l}0.4 \\
0.0\end{array}$ & $\begin{array}{l}297.0 \\
194.3\end{array}$ & $\begin{array}{l}0.2 \\
0.0\end{array}$ & $\begin{array}{l}0.7 \\
0.2\end{array}$ & $\begin{array}{l}123.4 \\
100.7\end{array}$ & $\begin{array}{l}91.9 \\
72.4\end{array}$ & $\begin{array}{l}0.0 \\
0.0\end{array}$ & & & $\begin{array}{l}0.9 \\
0.0\end{array}$ & 3 & & $\sigma$ & 0.0 & & $\begin{array}{l}3.2 \\
7.6\end{array}$ & & & & $\begin{array}{l}110.3 \\
138.7\end{array}$ \\
\hline $75-2,91-94$ & Sap. basalt; anal., aphyric & (?B) & 8.0 & 3.4 & 244.0 & 0.0 & 0.2 & 72.7 & 76.5 & 0.0 & 7.5 & 20.1 & 2.5 & 2 & 0.0 & 60 & 1.8 & 320.4 & 8.2 & 257.0 & .0 & $\begin{array}{l}92.9 \\
65.7\end{array}$ & 107.0 \\
\hline $76-1,10-13$ & Partly sap. basalt, anal.; oph & (?T) & 7.5 & 1.6 & 200.2 & 0.0 & 0.2 & 154.1 & 60. & 4.3 & 11.1 & 24.3 & 2.5 & 4. & 0.0 & 60 & 0.0 & 281.9 & 15.0 & 230.4 & 0.0 & 91.7 & 145.4 \\
\hline $76-2,67-70$ & Sap. basal & (B) & 12.1 & 5.0 & 194.7 & 0.0 & 0.0 & 55 & 71. & 0.0 & 10.1 & 18. & 0 & & 0 & 80 & 0 & 279.9 & 6.0 & 201.3 & & & 125.8 \\
\hline $76-2,117-120$ & Sap. basal & (?T) & 16.5 & 3.5 & 147.7 & 0.0 & 0.7 & 72.2 & 68 & 5.6 & 9.2 & 17.1 & 1.2 & -2 & 0 & 70 & 0.0 & 311.2 & 18.6 & 9 & 0.0 & & $\begin{array}{l}120.5 \\
171.7\end{array}$ \\
\hline $76-4,8-10$ & Sap. basalt; aphyric & (?) & 29.2 & 3.2 & 241.0 & 0.0 & 0.2 & 77.3 & 52.6 & 24.2 & 11.3 & 24.3 & 0.0 & 4.3 & 0.0 & 80 & 0.0 & 251.6 & 10.1 & 238.2 & 0.0 & 97.4 & 171.7 \\
\hline $76-5,22-24$ & $\begin{array}{l}\text { Partly sap. basalt; anal., } \\
\text { aphyric }\end{array}$ & (B) & 7.4 & 4.3 & 178.4 & 0.0 & 0.1 & 65.3 & 48.3 & 0.0 & 9.8 & 22.6 & 6.3 & 2.0 & 0. & 70 & 0.0 & 259.0 & 16.8 & 211.5 & 0.0 & 78.6 & 116.1 \\
\hline $77-4,7$ & $\begin{array}{l}\text { "Volcanic breccia" anal, } \\
\text { sap. }\end{array}$ & $\bar{z}$ & 8.5 & 3.6 & 62.3 & 0.0 & 0.2 & 85.1 & 86.0 & 25.9 & 13.9 & 33.7 & 4.9 & 3.5 & 0.0 & 110 & 0.0 & 264.0 & 0.0 & 44.4 & 0.7 & 64.2 & $\begin{array}{l}69.5 \\
62.9\end{array}$ \\
\hline $77-5,144-147$ & $\begin{array}{l}\text { sap. } \\
\text { "Volcanic b } \\
\text { sap. }\end{array}$ & $\bar{z}$ & $\begin{array}{r}9.4 \\
17.0\end{array}$ & 2.7 & $\begin{array}{l}168.4 \\
196.8\end{array}$ & $\begin{array}{l}0.0 \\
0.0\end{array}$ & $\begin{array}{l}0.0 \\
0.1\end{array}$ & $\begin{array}{l}81.8 \\
84.9\end{array}$ & $\begin{array}{l}51.9 \\
69.4\end{array}$ & $\begin{array}{l}23.8 \\
21.9\end{array}$ & $\begin{array}{l}13.8 \\
13.7\end{array}$ & $\begin{array}{l}32.0 \\
30.5\end{array}$ & 0.0 & 5.1 & 0.0 & 100 & 0.0 & $\begin{array}{l}295.0 \\
267.6\end{array}$ & 5.9 & $\begin{array}{l}46.6 \\
51.6\end{array}$ & $\begin{array}{l}0.0 \\
0.0\end{array}$ & $\begin{array}{l}59.6 \\
75.4\end{array}$ & 62 \\
\hline $78-2,97-100$ & $\begin{array}{l}\text { "Volcanic breccia" anal., } \\
\text { sap. }\end{array}$ & - & 16.0 & 2.2 & 163.2 & 0.0 & 0.0 & 79.0 & 41.8 & 34.7 & 11.8 & 27.2 & 0.0 & 2.7 & 0.0 & 100 & 0.0 & 221.1 & 3.4 & 45.2 & 0.0 & 67.8 & 75. \\
\hline $79-3,114-117$ & $\begin{array}{l}\text { "Volcanic breccia" anal., } \\
\text { sap. }\end{array}$ & - & 21.9 & 4.5 & 191.8 & 0.0 & 0.2 & 76.3 & 65.2 & 46.3 & 16.0 & 25.9 & 1.3 & 4.4 & 0.0 & 90 & 0.1 & 269.1 & 9.3 & 50.1 & 0.0 & 76.5 & 9 \\
\hline $80-2,80-83$ & $\begin{array}{l}\text { "Volcanic breccia" anal., } \\
\text { sap. }\end{array}$ & - & 23.4 & 2.7 & 207.1 & 0.0 & 0.7 & 66.0 & 73.0 & 40.8 & 12.9 & 41.6 & 0.0 & 4.5 & 0.0 & 110 & 3.5 & 385.5 & 12.8 & 53.3 & 0.0 & 95.8 & 103. \\
\hline $81-2,61-64$ & $\begin{array}{l}\text { "Volcanic breccia" anal., } \\
\text { sap. }\end{array}$ & - & 12.7 & 12.2 & 175.9 & 0.0 & 0.7 & 92.6 & 64.1 & 18.9 & 12.3 & 31.0 & 0.2 & 5.1 & 0.0 & 150 & 0.0 & 269.1 & 12.8 & 47.7 & 0.9 & 79.9 & 9 \\
\hline $81-2,133-136$ & $\begin{array}{l}\text { Partly sap. basalt, coarse, } \\
\text { aphyric }\end{array}$ & (T) & 6.7 & 20.7 & 292.2 & 0.6 & 0.3 & 97.8 & 71.3 & 0.0 & 17.1 & 30.0 & 5.0 & 3.3 & 0.0 & 90 & 1.2 & 327.7 & 28.6 & 113.6 & 0.0 & 90.7 & 14 \\
\hline $81-3,54-57$ & $\begin{array}{l}\text { Partly sap. basalt, dolerite, } \\
\text { oph. }\end{array}$ & (M) & 10.8 & 17.7 & 232.9 & 0.0 & 0.0 & 102.6 & 77.1 & 12.3 & 18.9 & 30.7 & 0.0 & 5.1 & 0.0 & 110 & 0.0 & 279.4 & 17.6 & 80.6 & 0.0 & 92.3 & 109. \\
\hline $81-3,1$ & $\begin{array}{l}\text { Partly sap. basalt, dolerite, } \\
\text { oph. }\end{array}$ & (M) & 9.5 & 17.1 & 24.9 & 0.4 & 0.3 & 114.2 & 62.0 & 0.0 & 18.3 & 28.2 & 4.8 & 4.6 & 0.0 & 90 & 2.1 & 331.8 & 30.6 & 80.0 & 0.0 & 72.8 & \\
\hline $82-1$ & a & & 4 & & & & & 3 & 112 & 7. & 14. & 34.3 & & & & & & .1 & & & & 98.8 & 11 \\
\hline & & & & 8 & & & & & & & & & & & & & & & & & & & \\
\hline & & & & 26. & & 0 & & 102 & & 0. & 11 & & & 4 & & & & 20 & & & & & 10 \\
\hline & & & & & & & & & & & 15 & & & & & 11 & & & & & & 102.6 & 147 \\
\hline & & & & & & 0 & 0 & & & 3. & 13 & & & & & & & & & & & 111.3 & 16 \\
\hline 90-1, & & (? & & & & 0. & & 12 & & 0. & & & & & & & & & & & & 83 & 91 \\
\hline 90-1, & basalt, coarse, & 0 & $\begin{array}{l}4.4 \\
4.5\end{array}$ & & & $\begin{array}{l}0 . \\
0 .\end{array}$ & $\begin{array}{l}0 . \\
0 .\end{array}$ & $\begin{array}{r}101 \\
99\end{array}$ & & $\begin{array}{l}8.5 \\
9.3\end{array}$ & $\begin{array}{l}15.2 \\
14.9\end{array}$ & & & & $\begin{array}{r}12 \\
0\end{array}$ & & & & & & 1 & $\begin{array}{r}111.8 \\
91.2\end{array}$ & \\
\hline 90-2, & basalt, coarse, & $c$ & 4.9 & 0. & 186.6 & 0. & 0.3 & 96.5 & 84.1 & 8.0 & 15.3 & 37. & 0.0 & 5.8 & 0 & 1 & .0 & 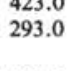 & 20.0 & 60.9 & $\begin{array}{l}0.1 \\
0.2\end{array}$ & $\begin{array}{r}91.2 \\
109.5\end{array}$ & $\begin{array}{r}80 \\
117\end{array}$ \\
\hline $91-1$, & $\begin{array}{l}\text { Partly sap. basalt, coarse, } \\
\text { aphyric }\end{array}$ & (T) & 6.2 & 1.1 & 196.5 & 0.0 & 0.7 & 108.9 & 51.2 & 0.6 & 17.7 & 35.3 & 3.7 & 6.0 & 0.0 & 100 & 17.0 & 361.2 & 21.1 & 60.4 & 0.0 & 98.9 & 108 \\
\hline & Sap. & (M) & 12 & & & & & & & & & & & & & & & & & & & & \\
\hline & Partly sap. basalt, aphyric & & & & & & & & & & & & & & & & & & & & & & \\
\hline & & & 5 & & & & & & & 13.3 & 14 & & & & 10 & & & & & & & ; & \\
\hline $96-2,95-98$ & Dolerite, olivine- & - & $5 . ?$ & & & & & & 107 & & & & & & & & & & & & & & \\
\hline & & & & & & & & & & & & & & & & & & & & & & & 13 \\
\hline , 36-39 & Microgabbro/coarse dolerite & - & & & & & & & & & & & & & & & & & & & & & \\
\hline & & & 0. & & & 0 & 0 & 9. & 8. & 0 & 18 & 35 & 12. & & 2 & & & 39 & & & & & \\
\hline $97-6,80-83$ & Dolerite, olivine- & - & 6 & & & & & & & & & & & & & & & & & & & & \\
\hline & & & & & & & & & & & & & & & & & & & & & & & \\
\hline & & & & & & & & & & & 15 & & & 7 & & 6 & & 8 & & & & 1 & 162 \\
\hline $96-5,108-111$ & Dolerite olivine & - & 5.4 & 0 & 218.8 & 0. & 0 & 91 & 122 & 16.8 & 14 & & & & & & & 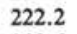 & & & & 6 & 137 \\
\hline & & & & & & & 0. & & & 15 & 14 & & & 4 & 10 & 11 & & & & & & 8 & \\
\hline $98-1,119-122$ & Dolerite, olivine & - & 5.5 & 0.7 & 209.6 & 5.0 & 0.0 & 86.1 & 60.9 & 3.6 & 13.7 & 36.9 & 0.0 & 4.6 & 1.8 & 90 & 0.5 & 230.7 & 22.8 & 305.5 & 0.0 & 119.9 & 148.2 \\
\hline
\end{tabular}


Table 19. Summary and comparison of whole-rock trace-element analyses of Holes 553A, 554A, and 555 (ppm).

\begin{tabular}{|c|c|c|c|c|c|c|c|c|c|c|c|c|c|}
\hline & & \multicolumn{2}{|c|}{ Hole 553A } & \multicolumn{2}{|c|}{ Hole $554 \mathrm{~A}$} & \multicolumn{2}{|c|}{ Hole 555} & \multicolumn{2}{|c|}{ Hole 555} & \multicolumn{2}{|c|}{$\begin{array}{c}\text { Hole } 553 \mathrm{~A} \\
\text { Hyaloclastites, } \\
\text { tuffs (4) }\end{array}$} & \multicolumn{2}{|c|}{$\begin{array}{c}\text { Hole } 555 \\
\text { Tuffs, } \\
\text { agglomerates (8) }\end{array}$} \\
\hline \multicolumn{2}{|c|}{ Group } & Mean & Range & Mean & Range & Mean & Range & Mean & Range & Mean & Range & Mean & Range \\
\hline \multirow{2}{*}{ IA } & $\mathrm{Li}$ & 5.32 & $1.5-9.3$ & 11.7 & $6.9-31.1$ & 8.4 & $4.4-29.2$ & 6.0 & $5.3-9.0$ & 10.1 & $3.9-18.1$ & 17.3 & $8.5-23.4$ \\
\hline & $\mathbf{R b}$ & 1.9 & $0.0-24.5$ & 5.8 & $1.5-11.1$ & 4.4 & $0.0-20.7$ & 1.2 & $0.2-2.9$ & 11.8 & $5.3-17.6$ & 7.3 & $2.2-26.6$ \\
\hline \multirow{3}{*}{ IB } & $\mathrm{Cu}$ & 206.0 & $10.15-676.5$ & 147.5 & $113.8-170.6$ & 232.6 & $147.7-327.1$ & 222.3 & $203.3-266.0$ & 182.8 & $51.9-101.2$ & 182.1 & $162.3-207.1$ \\
\hline & $\mathrm{Ag}$ & 0.2 & $0.0-1.5$ & 0.2 & $0-1.1$ & 0 & $0.0-0.6$ & 0 & $0.0-5.0$ & 0.04 & $0.0-0.2$ & 0 & 0 \\
\hline & $\mathrm{Be}$ & 0.4 & $0.0-1.3$ & 0.3 & $0.1-0.7$ & 3.2 & $0.0-0.7$ & 1.9 & $0.0-0.7$ & 0.5 & $0.0-1.0$ & 0.24 & $0-0.7$ \\
\hline \multirow[t]{2}{*}{ IIA } & $\mathrm{Sr}$ & 83.1 & $68.0-108.8$ & 92.6 & $87.8-99.9$ & 103.3 & $55.4-154.1$ & 88.8 & $83.9-94.1$ & 76.2 & $68.0-94.4$ & 83.5 & $66.0-92.6$ \\
\hline & $\mathrm{Ba}$ & 0.0 & 0.0 & 0.7 & $0.0-6.6$ & 21.0 & $0.0-161.6$ & 2.6 & $0.0-17.5$ & 1.3 & $0.0-5.6$ & 35.7 & $0-131.4$ \\
\hline \multirow{2}{*}{ IIB } & $\mathrm{Zn}$ & 79.7 & $49.7-139.2$ & 81.8 & $60.0-109.9$ & 81.5 & $7.5-130.1$ & 86.4 & $52.2-107.7$ & 84.8 & $59.0-160.2$ & 63.3 & $41.8-86.0$ \\
\hline & $\mathrm{Cd}$ & tr & $0.0-0.26$ & 0.0 & 0.0 & 0.0 & 0.0 & 0.0 & $0.0-5.0$ & 0.0 & 0.0 & 0.0 & 0 \\
\hline \multirow[t]{2}{*}{ IIIA } & B & 4.1 & $0.0-17.0$ & 16.4 & $0-34.5$ & 3.8 & $0-24.2$ & 8.8 & $0-17.8$ & 28.1 & $0.0-60.0$ & 26.5 & $18.9-46.3$ \\
\hline & $\mathrm{Ga}$ & 13.9 & $8.4-17.5$ & 15.3 & $14.2-17.1$ & 14.9 & $7.5-18.9$ & 15.1 & $13.7-18.7$ & 11.1 & $9.9-15.0$ & 13.2 & $11.8-16.0$ \\
\hline \multirow{2}{*}{ IIIB } & $\mathrm{Y}$ & 31.3 & $9.5-49.6$ & 28.4 & $23.6-33.9$ & 29.0 & $17.1-49.5$ & 32.1 & $22.2-36.9$ & 31.1 & $18.4-59.4$ & 31.6 & $25.9-41.6$ \\
\hline & $\mathrm{La}$ & 3.4 & $0.0-17.2$ & 2.7 & $0.0-12.5$ & 1.1 & $0.0-6.3$ & 1.1 & $0-12.9$ & 2.4 & $0.0-8.3$ & 1.6 & $0-4.9$ \\
\hline \multirow{2}{*}{ IVA } & Sn & 4.7 & $0.0-7.9$ & 5.0 & $1.4-8.8$ & 4.4 & $1.0-7.2$ & 5.2 & $1.2-8.4$ & 7.8 & $3.0-11.6$ & 4.0 & $2.7-8.1$ \\
\hline & $\mathrm{Pb}$ & 2.7 & $0.0-13.9$ & 7.4 & $0.0-13.0$ & 1.8 & $0.0-12.7$ & 11.6 & $1.8-30.2$ & 0.0 & $0.0-17.6$ & 0.0 & 0 \\
\hline IVB & $\mathrm{Zr}$ & 112 & $70 .-180$ & 110 & $80-130$ & 110 & $60-170$ & 110 & $60-110$ & 114 & $44.9-170$ & 100 & $90-150$ \\
\hline VA & $\mathrm{Bi}$ & 2.9 & $0.0-8.7$ & 2.2 & $0.8-3.7$ & 1.2 & $0.0-17.0$ & 1.3 & $0-4.2$ & 1.8 & $0.0-4.5$ & 0.6 & $0-3.5$ \\
\hline \multirow{2}{*}{ VB } & v & 316.0 & $178.7-483.6$ & 311.3 & $217.3-387.8$ & 306.5 & $224.3-443.8$ & 268.8 & $210.8-343.5$ & 227.0 & $163.1-260.0$ & 271.8 & $221.0-385.5$ \\
\hline & $\mathrm{Nb}$ & 21.2 & $5.9-40.2$ & 31.8 & $18.1-47.9$ & 17.6 & $7.0-33.2$ & 26.0 & $12.9-34.1$ & 11.0 & $3.4-14.8$ & 6.4 & $0-12.8$ \\
\hline \multirow{2}{*}{ VIB } & $\mathrm{Cr}$ & 83.9 & $21.0-257.7$ & 91.8 & $34.0-224.0$ & 130.1 & $60.4-257.0$ & 212.3 & $25.6-305.5$ & 36.7 & $20.3-83.0$ & 49.2 & $44.4-53.3$ \\
\hline & Mo & 1.4 & $0.0-7.9$ & 0.9 & $0.0-3.7$ & 0.4 & $0.0-6.1$ & 0.6 & $2.0-2.7$ & 2.6 & $0.0-6.8$ & 0.2 & $0-0.9$ \\
\hline \multirow[t]{2}{*}{ VIII } & Co & 117.1 & $10.15-526.6$ & 122.5 & $85.6-189.1$ & 89.4 & $65.7-111.8$ & 72.6 & $70.2-122.2$ & 85.3 & $51.9-91.0$ & 106.2 & $59.6-95.8$ \\
\hline & $\mathrm{Ni}$ & 117.5 & $69.7-175.2$ & 101.8 & $84.0-142.1$ & 122.7 & $73.9-171.7$ & 125.8 & $53.0-162.0$ & 104.8 & $62.1-158.0$ & 81.9 & $62.9-103.8$ \\
\hline
\end{tabular}

Note: Analysts: K. A. Holmes, P. Joseph, and V. A. Judge, Analytical Chemistry Unit, Institute of Geological Sciences.

Table 20. Rare-earth element abundances in selected samples from Holes 553A, 554A, and 555 .

\begin{tabular}{lccccccccc}
\hline \multirow{2}{*}{$\begin{array}{c}\text { Sample } \\
\text { (interval in cm) }\end{array}$} & \multicolumn{8}{c}{ Rare-earth elements (ppm) } \\
\cline { 2 - 10 } & $\mathrm{La}$ & $\mathrm{Ce}$ & $\mathrm{Nd}$ & $\mathrm{Sm}$ & $\mathrm{Eu}$ & $\mathrm{Tb}$ & $\mathrm{Ho}$ & $\mathrm{Yb}$ & $\mathrm{Lu}$ \\
\hline 553A-40-1, 79-82 & 1.8 & 13.6 & 8.5 & 3.92 & 1.67 & 0.89 & 1.7 & 3.96 & 0.71 \\
$553 \mathrm{~A}-55-6,44-47$ & 1.1 & 8.7 & 4.7 & 2.54 & 0.88 & 0.54 & 1.2 & 2.68 & 0.44 \\
$553 \mathrm{~A}-58-2,117-120$ & 0.95 & $<2.4$ & 4.0 & 2.47 & 0.86 & 0.72 & 1.32 & 3.58 & 0.59 \\
$554 \mathrm{~A}-7-3,10-12$ & $<1.1$ & $<3.1$ & 5.0 & 2.39 & 1.94 & 0.35 & 0.96 & 2.01 & 0.37 \\
$554 \mathrm{~A}-14-1,36-39$ & $<1.0$ & $<2.8$ & 6.5 & 2.45 & 1.40 & 0.43 & 0.95 & 2.17 & 0.37 \\
$555-81-2,133-136$ & 1.4 & $<2.5$ & 4.0 & 2.45 & 0.83 & 0.54 & 0.97 & 2.55 & 0.41 \\
555-96-5, 85-88 & $<1.0$ & $<2.6$ & 3.7 & 2.20 & 1.07 & 0.47 & 0.94 & 2.13 & 0.39 \\
Errors based on & $10-20 \%$ & $5 \%$ & $10 \%$ & $2 \%$ & $5 \%$ & $10 \%$ & $3 \%$ & $3 \%$ & $3 \%$ \\
$\quad$ counting statistics & & & & & & & & & \\
\hline
\end{tabular}

Note: Analyst: Dr. Susan Parry, University of London (Imperial College) Reactor Center.

basalts (except two coarse-grained samples) and the volcaniclastic rocks have zero or negligible $\mathrm{Pb}$. In the olivine-dolerites the $\mathrm{Pb}$ ranges up to $30.2 \mathrm{ppm}$ which is near the average for granites in which $\mathrm{Pb}$ is normally enriched (Rankama and Sahama, 1950).

\section{$\mathrm{Zr}$}

Zirconium, with the exception of seven variably saponitized basalts and two olivine-dolerites, varies relatively little throughout the succession, and there is no systematic relation to lithology or other factors. The mean is similar for basalts and volcaniclastics and to the other sites. $\mathrm{Zr}$ correlates positively with $\mathrm{Y}$ for basalts and dolerites (Fig. 13), and closely correlates with basalts from Hole 553A.

$\mathrm{Bi}$

Bi levels are nil or very low, but with sporadic values up to $17.0 \mathrm{ppm}$ for a basalt near a flow top.

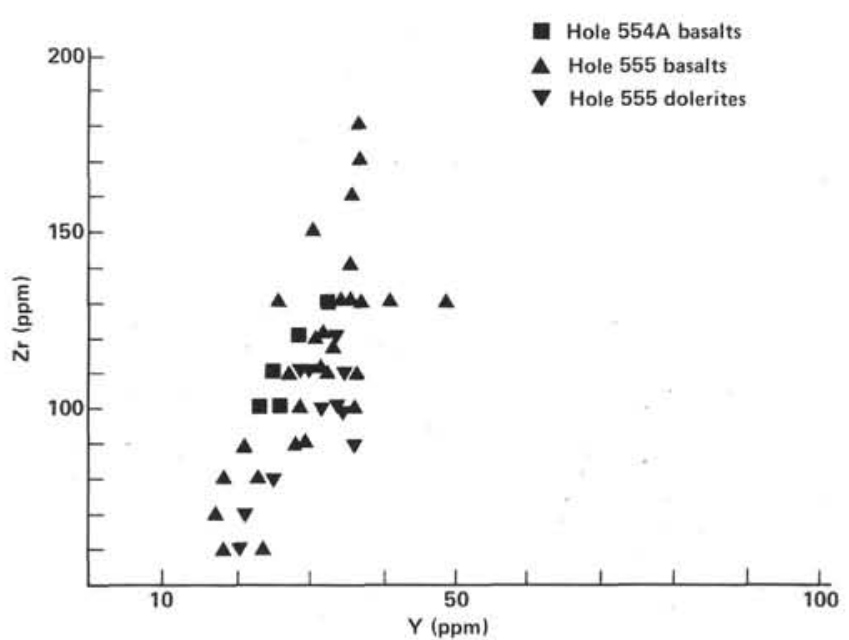

Figure 13. Zr:Y diagram for basalts from Sites 554 and 555 and for dolerites from Site 555 . 


\section{$\mathrm{V}, \mathrm{Nb}$}

The plot of $\mathrm{Nb} / \mathrm{V}$ (Fig. 14) shows a considerable scatter for the basalts, but the olivine-dolerites with two exceptions plot linearly; the line is displaced from that for the basalts but the two are approximately parallel. The analyzed basalts of Hole 554A scatter about the olivine-dolerite plot. Allowing for scatter, the correlation of the Hole 555 basalts approximately coincides in slope and position with that for the Hole 553A basalts (Fig. 12). The volcaniclastic rocks contain subaverage $\mathrm{Nb}$, some below the limit of detection.

\section{$\mathrm{Cr}, \mathrm{Mo}$}

Molybdenum values are very small or below detection. $\mathrm{Cr}$ is enriched in some basalts, as in Samples 555-75-2, 91-94 cm and 555-76-1, 10-13 cm, depleted especially in the volcaniclastic rocks, but enriched again in the olivine-dolerites except in a micrograbbro in Sample 555-97-3, 36-39 cm which is also depleted in Co and Ni. The microgabbro does not contain unaltered olivine, and there are only smectitic pseudomorphs after possible olivine. No spinels were observed. There is much unaltered clinopyroxene, and the reason for the depletion in $\mathrm{Co}$ and $\mathrm{Ni}$ is not apparent unless this represents a separate intrusion petrogenetically unrelated to the dol-

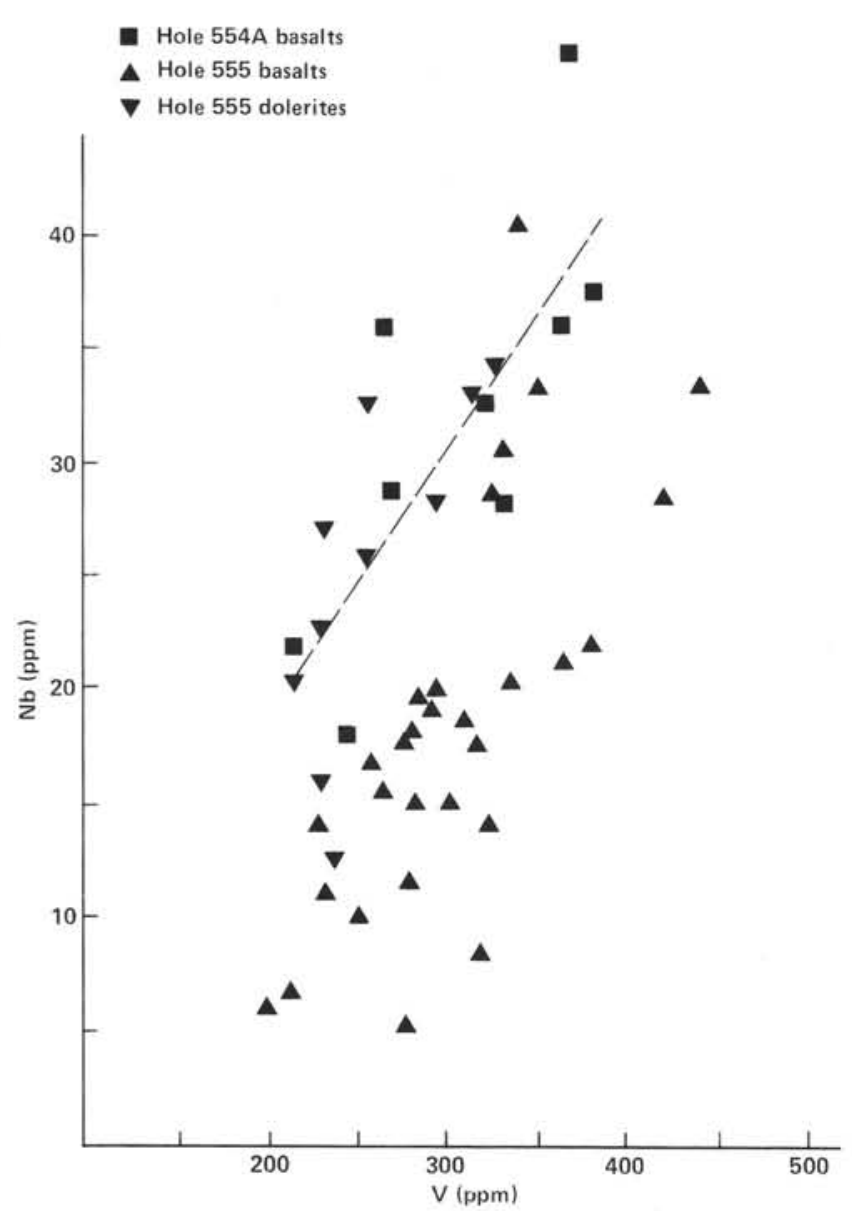

Figure 14. $\mathrm{Nb}: \mathrm{V}$ diagram for basalts from Holes $554 \mathrm{~A}$ and 555 and for dolerites from Hole 555 . erites. The mean $\mathrm{Cr}$ content of the other olivine-dolerites is considerably greater than those of the basalts and volcaniclastics of the three holes.

$\mathrm{Co}, \mathrm{Ni}$

Both elements show relative enrichment in the olivine-dolerites of Unit Vc, but are depleted in the microgabbro in Sample 555-97-3, 36-39 cm. There is comparable enrichment of $\mathrm{Ni}$ in some of the basalts and depletion in the volcaniclastic rocks. The mean Co value for the basalts is lower than that for Holes 553A and 554A, but the mean Ni contents are similar for all three sites. The mean Co value of the olivine-dolerites is similar to that of the Hole 553A basalts and also the volcaniclastic rocks.

\section{Rare-Earth Elements (REE)}

The results (Table 20) show that, with the main exception of $\mathrm{Ce}$, the rare earths are of similar orders for samples from Sites 553, 554, and 555-except for the highest, labradorite-clinopyroxene-microphyric basalt (Sample $553 \mathrm{~A}-40-1,79-82 \mathrm{~cm}$ ), which is, relative to the other labradorite-microphyric basalt, enriched especially in the light REE (LREE) Ce, Nd, and Sm. Even so, all samples are "normal" LREE depleted with $\mathrm{La}$ /Sm and $\mathrm{La} / \mathrm{Yb}$ ratios less than 1.0 , similar to the Type 1 basalts of Bryan et al. (1976). The two Site 554 labradorite-microphyric basalts show marked positive Eu anomalies and depletion in $\mathrm{Tb}$. The olivine-dolerite and aphyric basalt samples from Site 555 are closely similar except for a slight positive Eu anomaly in the former.

The analyses fall within the range of values for the heavier REE of the Mid-Atlantic Ridge (Frey et al., 1974; Emmermann and Puchett, 1979), but the Leg 81 values for the LREE ( $\mathrm{La}, \mathrm{Ce}, \mathrm{Nd}$ ) are mainly lower. With these notable exceptions, the Leg 81 REE patterns generally agree with those of the phyric basalts of Legs 45 and 46.

The chondrite-normalized plots (Fig. 9) generally show the convex-up characteristic of MORB and ocean-floor basalts (Frey et al., 1968), with depletion in the LREE typical of tholeiites (subalkaline basalts). This depletion has been attributed by those authors and others (e.g., Haskin, 1979), to partially melted mantle, itself a residuum from mantle depleted in incompatible elements from a previous melting event.

\section{Initial Strontium-Isotope Ratios}

Two basalts, one each from Holes 553A and 554A, and an olivine-dolerite from Hole 555 were analyzed, mainly to determine whether the magmas had been contaminated by continental crust. The results (Table 21) gave low initial $\mathrm{Sr}$ ratios indicating no such contamination. The ratio for the olivine-dolerite is significantly lower than for the basalts, indicating that the former represents a more primitive source.

\section{SECONDARY ALTERATION}

\section{Mineralogy and Petrography}

All of the rocks examined show some degree of alteration (Fig. 15), mainly to clay minerals, those of the smectite group and Fe-micas of the celadonite type be- 
Table 21. Initial strontium isotope ratio analyses ${ }^{\mathrm{a}}$ of basalts from Holes $553 \mathrm{~A}$ and $554 \mathrm{~A}$ and of dolerite from Hole 555 .

\begin{tabular}{|c|c|c|c|c|c|c|}
\hline $\begin{array}{c}\text { Sample } \\
\text { (interval in } \mathrm{cm} \text { ) }\end{array}$ & Lithology & $\begin{array}{c}\mathrm{Rb} \\
(\mathrm{ppm})\end{array}$ & $\underset{(\mathrm{ppm})}{\mathrm{Sr}}$ & ${ }^{87} \mathrm{Rb} /{ }^{86} \mathrm{Sr}$ & $\begin{array}{c}{ }^{87} \mathrm{Rb} /{ }^{86} \mathrm{Sr} \\
\text { (meas.) }\end{array}$ & $\begin{array}{c}{ }^{87} \mathrm{Sr} /{ }^{86} \mathrm{Sr} \\
\text { (initial }\end{array}$ \\
\hline $553 \mathrm{~A}-59-2,115-118$ & $\begin{array}{l}\text { Feldspar-microphyric } \\
\text { basalt }\end{array}$ & 0.2 & 88.7 & 0.0072 & $0.70367 \pm 7$ & $0.70366 \pm 7$ \\
\hline $554 \mathrm{~A}-7-3,10-12$ & $\begin{array}{l}\text { Feldspar-clinopyroxene- } \\
\text { microphyric basalt }\end{array}$ & 2.9 & 92.0 & 0.0911 & $0.70374 \pm 7$ & $0.70367 \pm 7$ \\
\hline $555-96-5,108-111$ & Olivine-dolerite & 0.8 & 91.5 & 0.0252 & $0.70382 \pm 7$ & $0.70308 \pm 7$ \\
\hline
\end{tabular}

a Analyst Jane Evans.

b Recalculated assuming an age of $55 \pm 10$ m.y. (1-sigma). Errors on ${ }^{87} \mathrm{Sr} /{ }^{86} \mathrm{Sr}$ are quoted for the fifth decimal place; all the results are within the same range of error.

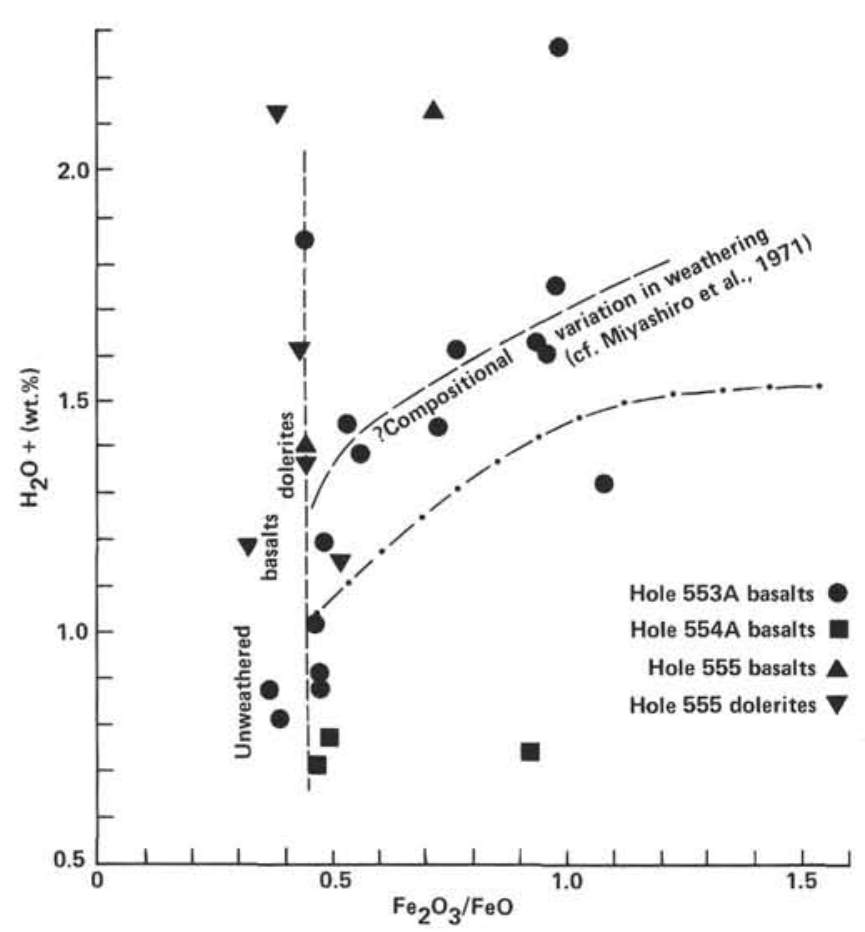

Figure 15. Variation of oxidation $\left(\mathrm{Fe}_{2} \mathrm{O}_{3} / \mathrm{FeO}\right)$ with hydration $\left(\mathrm{H}_{2} \mathrm{O}^{+}\right)$ in Leg 81 basalts and dolerites.

ing most commonly present (Tables 1 and 2 ). Smectites are represented mainly by saponite, but minor amounts of nontronite and Fe-montmorillonite are also present. On XRD traces, saponite is identified by air-dried basal spacings (001) of 12.5-15 $\AA$ which expand to $17.7 \AA$ with glycerol saturation; 060 spacings of $1.535-1.540 \AA$ characterize the trioctahedral nature of this mineral. Electron microprobe analyses show that saponite with sodium the dominant interlayer cation is common in rocks from Sites 553 and 555 (Table 22). Na-saponites give air-dried basal spacings of $12.5-13 \AA$, in contrast with 14-15 $\AA$ spacings shown by Ca-saponites also occurring in the samples. XRD analyses of celadonites show that they are dioctahedral, $1 \mathrm{M}$ mica polytypes with basal spacings of 9.8-10.0 $\AA$. Analysis by electron microprobe indicates that the octahedral trivalent cations total $\Sigma^{3+}$ is $\sim 1.0$ in structural formula (Table 23 ), which is compatible with celadonite rather than glauco nite, using the criteria of Buckley et al. (1978). The opti- cal distinction between saponite and celadonite is most obvious in some of the more heavily altered vesicular basalts from Holes 553A and 555. Here celadonite occurs as subradiating clusters of pleochroic blue-green needles up to $0.15 \mathrm{~mm}$ in length. Saponites mostly show brown colors, ranging from pleochroic, colorless to pale brown microscopic flakes (up to $0.15 \mathrm{~mm}$ ), to nonpleochroic, reddish brown (Fe stained?), submicroscopic intergrowths. Maximum birefringence shown by pale-brown saponite flakes is second-order blue or green $(0.012-$ 0.013 ); celadonite birefringence is lower, but masked by the strong blue-green color.

In basalts from Hole 553A, the greatest degree of alteration occurs in the vesicular or scoriaceous tops of flows. Saponite replaces interstitial glass, olivine, and clinopyroxene, while infilled vesicles typically show saponitic margins and celadonitic centers. Some plagioclases microphenocryts may be replaced by saponite, and in the most heavily altered basalts, groundmass plagioclase is also partially replaced by saponite. In the least altered basalts, generally the nonvesicular portions of flows, argillization is confined to interstitial glass which is completely replaced by brown or greenish-brown saponite. The latter commonly possesses a rim of palegreen celadonite, suggesting that celadonite is forming at the expense of saponite. Calcite occurs sparingly in the basalts and mostly as a late (post-argillization?) vesicle filling. Heavy alteration characterizes the pyroclastic horizons in Hole 553A, with $\mathrm{Na}$ - and $\mathrm{Ca}$-saponites the main secondary minerals. Sample 533A-45-1, 87-91 $\mathrm{cm}$ shows glass altering at clast margins and vesicle walls to a fringe of tiny ( $6 \mu \mathrm{m})$ flakes of aluminous smectite (Fe montmorillonite?), passing into coarser intergrowths of saponite and celadonite forming the matrix of the tuff. Internally, glass clasts show little evidence of argillization, but the orange-red glass is strongly hematitized, containing up to $25.80 \% \mathrm{FeO}$ by microprobe analyses. The petrographic evidence suggests that oxidation, probably accompanied by leaching of $\mathrm{Ca}$ and alkalies, was accomplished prior to argillization of the tuff.

Clay mineral alteration is generally less severe in samples from Hole 554A. The dominant secondary mineral is a yellow-green, random mixed-layer celadonite/saponite which replaces interstitial glass, olivine, and, less commonly, pyroxene. The same mineral occurs in vesicles as yellow-brown, structureless, or radially microfi- 
Table 22. Representative saponite analyses (Holes 552, 553A, 554A, and 555) normalized to eleven oxygens.

\begin{tabular}{lrrrrrr}
\hline & \multicolumn{7}{c}{ Sample (interval in cm) } \\
\cline { 2 - 7 } & $\begin{array}{c}553 \mathrm{~A}-43-1, \\
20-23\end{array}$ & $\begin{array}{c}553 \mathrm{~A}-46-1, \\
121-125\end{array}$ & $\begin{array}{c}553 \mathrm{~A}-55-3, \\
113-116\end{array}$ & $\begin{array}{c}555-69-3, \\
113-116\end{array}$ & $\begin{array}{r}555-81-3, \\
110-113\end{array}$ & $\begin{array}{r}555-95-1, \\
97-100\end{array}$ \\
\hline $\mathrm{SiO}_{2}$ & 45.97 & 45.92 & 46.61 & 47.14 & 43.10 & 46.59 \\
$\mathrm{Al}_{2} \mathrm{O}_{3}$ & 5.76 & 4.79 & 5.50 & 4.11 & 8.00 & 3.34 \\
$\mathrm{Fe}_{2} \mathrm{O}_{3}$ & 0.05 & 0.05 & 0.05 & 0.01 & 0.05 & 2.25 \\
$\mathrm{FeO}$ & 16.89 & 20.68 & 16.84 & 21.09 & 22.24 & 16.30 \\
$\mathrm{MnO}$ & 0.13 & 0.13 & 0.03 & 0.08 & 0.06 & 0.26 \\
$\mathrm{MgO}$ & 15.42 & 13.67 & 16.87 & 13.29 & 12.78 & 16.96 \\
$\mathrm{TiO}$ & 0.0 & 0.29 & 0.07 & 0.44 & 0.10 & 0.04 \\
$\mathrm{CaO}$ & 0.48 & 0.72 & 1.49 & 0.92 & 2.53 & 0.97 \\
$\mathrm{Na}{ }_{2} \mathrm{O}$ & 2.28 & 1.65 & 1.27 & 1.92 & 1.86 & 1.84 \\
$\mathrm{~K}_{2} \mathrm{O}$ & 0.85 & 0.27 & 0.02 & 0.14 & 0.13 & 0.08 \\
$\mathrm{Total}$ & 87.83 & 88.17 & 88.75 & 89.14 & 90.85 & 88.63 \\
& & & & & & \\
$\mathrm{Si}$ & 3.55 & 3.58 & 3.53 & 3.64 & 3.32 & 3.57 \\
$\mathrm{Al}$ & 0.45 & 0.42 & 0.47 & 0.36 & 0.68 & 0.30 \\
$\mathrm{Fe}$ & 0.00 & 0.00 & 0.00 & 0.00 & 0.00 & 0.13 \\
$\mathrm{Al}$ & 0.07 & 0.02 & 0.02 & 0.01 & 0.05 & 0.00 \\
$\mathrm{Fe}$ & 1.09 & 1.35 & 1.07 & 1.36 & 1.43 & 1.04 \\
$\mathrm{Mn}$ & 0.01 & 0.01 & 0.00 & 0.01 & 0.00 & 0.02 \\
$\mathrm{Mg}$ & 1.77 & 1.59 & 1.91 & 1.53 & 1.47 & 1.94 \\
$\mathrm{Ti}$ & 0.00 & 0.02 & 0.00 & 0.03 & 0.01 & 0.00 \\
$\mathrm{Ca}$ & 0.04 & 0.06 & 0.12 & 0.08 & 0.21 & 0.08 \\
$\mathrm{Na}$ & 0.34 & 0.25 & 0.19 & 0.29 & 0.28 & 0.27 \\
$\mathrm{~K}$ & 0.08 & 0.03 & 0.00 & 0.01 & 0.01 & 0.01 \\
$\mathrm{IA}$ & 0.46 & 0.34 & 0.31 & 0.38 & 0.50 & 0.36 \\
\hline
\end{tabular}

Table 23. Representative celadonite analyses (Hole 533A) and a chlorite analysis (Hole 555).

\begin{tabular}{|c|c|c|c|c|}
\hline & \multicolumn{4}{|c|}{ Sample (interval in $\mathrm{cm}$ ) } \\
\hline & $\begin{array}{c}\text { 553A-39-1, } \\
2-4 \\
\text { Celadonite }\end{array}$ & $\begin{array}{c}553 \mathrm{~A}-43-1, \\
20-23 \\
\text { Celadonite }\end{array}$ & $\begin{array}{c}553 \mathrm{~A}-52-3, \\
140-143 \\
\text { Celadonite }\end{array}$ & $\begin{array}{l}555-18-3, \\
110-113 \\
\text { Chlorite }\end{array}$ \\
\hline $\mathrm{SiO}_{2}$ & 54.63 & 55.45 & 51.60 & 30.18 \\
\hline $\mathrm{Al}_{2} \mathrm{O}_{3}$ & 3.56 & 3.25 & 4.06 & 12.30 \\
\hline $\mathrm{Fe}_{2} \mathrm{O}_{3}$ & 14.00 & 14.50 & 14.50 & 0.00 \\
\hline $\mathrm{FeO}$ & 4.83 & 5.44 & 4.30 & 34.77 \\
\hline $\mathrm{MnO}$ & 0.10 & 0.08 & 0.01 & 0.10 \\
\hline $\mathrm{MgO}$ & 6.72 & 6.29 & 8.68 & 10.14 \\
\hline $\mathrm{TiO}_{2}$ & 0.04 & 0.12 & 0.00 & 0.00 \\
\hline $\mathrm{CaO}$ & 0.18 & 0.09 & 0.70 & 0.68 \\
\hline $\mathrm{Na}_{2} \mathrm{O}$ & 0.60 & 0.53 & 0.92 & 0.50 \\
\hline $\mathrm{K}_{2} \mathrm{O}$ & 9.67 & 10.27 & 7.22 & 0.00 \\
\hline Total & 94.33 & 96.02 & 91.99 & 88.6 \\
\hline No. of Os & 11 & 11 & 11 & $28^{\mathrm{a}}$ \\
\hline $\mathrm{Si}$ & 3.94 & 3.95 & 3.80 & 6.62 \\
\hline $\mathrm{Al}_{\mathrm{v}}^{\mathrm{IV}}$ & 0.06 & 0.05 & 0.20 & 1.38 \\
\hline $\mathrm{Al}^{\mathrm{VI}}$ & 0.24 & 0.22 & 0.15 & 1.80 \\
\hline $\mathrm{Fe}^{3+}$ & 0.76 & 0.78 & 0.80 & 0.00 \\
\hline $\mathrm{Fe}^{2+}$ & 0.29 & 0.32 & 0.27 & 6.38 \\
\hline $\mathrm{Mn}$ & 0.01 & 0.01 & 0.00 & 0.02 \\
\hline $\mathrm{Mg}$ & 0.72 & 0.69 & 0.95 & 3.32 \\
\hline $\mathrm{Ti}$ & 0.00 & 0.01 & 0.00 & 0.00 \\
\hline $\mathrm{Ca}$ & 0.01 & 0.01 & 0.06 & 0.16 \\
\hline $\mathrm{Na}$ & 0.08 & 0.07 & 0.13 & 0.21 \\
\hline $\mathrm{K}$ & 0.89 & 0.93 & 0.68 & 0.00 \\
\hline$\Sigma\left(R_{2+}^{3+}\right)$ & 1.06 & 1.00 & 0.95 & 1.80 \\
\hline$\Sigma\left(\mathrm{R}^{3+}+\mathrm{R}^{2+}\right)$ & 2.02 & 2.03 & 2.17 & 11.89 \\
\hline$\Sigma \mathrm{A}$ & 0.988 & 1.01 & 0.87 & - \\
\hline
\end{tabular}

${ }^{a}$ Assume $16(\mathrm{OH})$ for chlorite.

brous clay (e.g., Sample 554A-9-1, 75-78 cm), although some vesicles are calcite filled, with traces of hematite, as in Samples 554A-7-4, 25-28 cm and 554A-8-1, 99-102 $\mathrm{cm}$. Discrete celadonite occurs as pale-green, poorly birefringent material replacing interstitial glass.
The greatest variety of secondary minerals is found at Site 555. In addition to saponite and celadonite, analcime, calcite, hematite, chalcedony, serpentine, chlorite, and amphibole are present, usually in minor amounts. Calcite appears to be an early alteration of olivine in some basalts, commonly forming the core of saponite or, rarely, serpentine pseudomorphs; it also replaces what appears to have been interstitial glass, but is invariably altered to saponite on the margins of such interstices. However, interstitial glass in the basalts is most commonly replaced by saponite-celadonite intergrowths; in more heavily altered basalt, saponite also replaces cores of plagioclase microphenocrysts. In many vesicles there is an overall zonation of minerals: smectite commonly forms rims, as dark brown or yellow-brown structureless to microfibrous aggregates. The centers may be filled with calcite (fibrous or sparry), radially fibrous celadonite, chalcedony (also radially fibrous nucleated from the $\mathrm{rim})$, and/or analcime. It is difficult to distinguish optically between microfibrous smectite and celadonite in some of the samples, but elsewhere celadonite tends to occupy the center of vesicles surrounded by smectite. In the same core (e.g., Sample 554A-76-1, 10-13 cm) smectite may rim radially fibrous calcite or celadonite or chalcedony. In Sample 554A-76-5, 22-24 cm, smectite rims radially fibrous chalcedony, which in turn projects into analcime.

Intergrowths of pale-green chlorite and saponite are found in Hole 555 samples, mostly as subradiating infilling of vesicles in the basalts and also in spherulitic basaltic glass (Sample 555-76-1, 117-120 cm). Occasionally the low birefringence of chlorite distinguishes it from saponite, but intergrowths are usually too fine to allow optical identification. Analcime and saponite are the main secondary minerals in the hyaloclastites cored at Site 555; glass fragments are pale green or yellow green due 
to replacement by saponite, but remain poorly birefringent (Table 2). Outgrowths of tiny saponite flakes (up to $4 \mu \mathrm{m}$ ), fringing the glass, are brightly birefringent (max. 0.018 ), in contrast to the poorly anisotropic, pale-brown analcime that forms the matrix of the tuff. Analcime appears to be a late alteration product of tuffs in Core $82-2,74-81 \mathrm{~cm}$, where it partially replaces saponitechlorite intergrowths filling vesicles. Alteration of the dolerites cored in Hole 555 is commonly centered on interstitial areas, possibly representing original microcrystalline material or a glassy mesostasis. These areas may show well-defined zones of secondary minerals; brown, sub-microcrystalline saponite and celadonite intergrowths form an outer zone, followed inward by subradiating flakes (up to $0.08 \mathrm{~mm}$ ) of pale bluish-green $\mathrm{Fe}$ chlorite (ripidolite; analysis, Table 23), which toward the center of such areas are increasingly intergrown with brownishgreen radiating flakes of saponite. Electron microprobe analyses show that saponites contain more $\mathrm{Mg}$ and $\mathrm{Na}$ in the centers of zoned alteration, but contain less $\mathrm{Fe}$, $\mathrm{Al}$, and $\mathrm{Ca}$. Extension of these areas has enclosed more coarsely crystalline primary mineral, which can be seen forming ghosts in the phyllosilicate intergrowths. Analcime forms a later replacement of the more extensive areas of alteration (up to $5 \mathrm{~mm}$ in length) in the dolerites. Outside of these areas, selective replacement of primary minerals may result in fresh pyroxene ophitically enclosing partially saponitized plagioclase. Amphibole is present in two dolerite samples from Hole 555 (Samples 555-95-1, 97-100 cm and 555-96-2, 95-98 cm). It occurs as sprays of colorless fibers most commonly intergrown with interstitial saponite and chlorite but here and there forms outgrowths on clinopyroxenes. Fibers are too slender $(<2 \mu \mathrm{m})$ for electron microprobe analysis; they are probably an actinolitic amphibole.

\section{Conditions of Alteration}

Significant variations occur in secondary mineral assemblages found in the basaltic rocks of the three sites studied. Much of the alteration in Hole 553A is typically open space mineralization of the vesicular upper and lower portions of flows, accompanied by replacement of interstitial glass. With the exception of olivine, primary minerals are little altered and least altered in the centers of flows. A typical secondary mineral assemblage in Hole 553A lavas is saponite-celadonite-minor calcite-trace hematite. Textural evidence suggests that saponite is the first mineral to form, initially by hydrolyzation of glass and subsequently by precipitation in vesicles. Vesicularity and degree of alteration are closely linked, and there is little doubt that inherent porosity was the main means of access of the water necessary for alteration. The Mg-rich nature of the saponite formed and the dominance of $\mathrm{Na}^{+}$over $\mathrm{Ca}^{2+}$ in interlayer sites in this mineral suggests that seawater was responsible for much of the alteration. Celadonite appears to form at the expense of early saponite and must reflect increasingly oxidative conditions of alteration, presumably as a result of continued passage of seawater. The occurrence of minor amounts of calcite as a late vesicle filling in Hole 553A basalts suggests that the saline fluids eventu- ally became $\mathrm{Ca}$ rich in the presence of a partial pressure of $\mathrm{CO}_{2}$. The latter may be linked with decreasing permeability of progressively argillized flows, both conditions apparently inhibiting the development of zeolites.

Andrews (1980) describes celadonite replacing saponite formed by the alteration of basalts from the FAMOUS area. He concludes that celadonite develops in response to falling temperatures caused by the passage of seawater through initial warm rock which he estimates was at temperatures of $<150^{\circ} \mathrm{C}$ when saponite formed. For hydrothermally altered Icelandic tholeiites, Kristmannsdottir (1978) reports increasing interlayering of smectites below $200^{\circ} \mathrm{C}$ and progressive transformation to chlorite at $200-240^{\circ} \mathrm{C}$. The absence of interlayered or discrete chlorite in Hole 553A basalts suggests alteration temperature below $200^{\circ} \mathrm{C}$ and possibly similar to those estimated by Andrews (1980), namely $<150^{\circ} \mathrm{C}$.

Secondary mineral assemblages in Hole 554A are broadly similar to those found in Hole 553A. The main difference appears to be the dominance of celadonite and the occurrence of a mixed-layer celadonite/saponite mineral instead of discrete saponite. Prolonged, lowtemperature seawater alteration is indicated by the celadonite-rich assemblages.

Three assemblages of secondary minerals occur in the basaltic rocks of Site 555. The topmost part of Subunit IVa contains essentially the same suite as that in Hole $553 \mathrm{~A}$, that is, saponite-celadonite-minor calcite. Hematite and late analcime appear in Sample 555-75-2, 91-94 cm and are common secondary minerals throughout the middle and low part of Subunit IVa, but are absent from Subunits IVb and IVc. Chlorite appears in the middle of Subunit IVa (Sample 555-76-2, 117-120 cm) and occurs sporadically throughout this subunit and Subunit IVb, but is persistent in the dolerites of Subunit IVc. Sparse amphibole is also present in Subunit IVc. Thus a typical secondary mineral assemblage in the middle and lower parts of Subunit IVa is: saponite-celadonite-analcime-hematite-chlorite, whereas in Subunits IVb and IVc saponite-celadonite-chlorite-sparse amphibole is typical. These changes in mineral assemblage indicate that alteration in the deepest rocks penetrated at Site 555 occurred under greenschist-facies condition, while those in the upper part of the site suffered zeolitefacies alteration, the transition zone lying within Subunit IVb. Chlorite-bearing assemblages appear in hydrothermally altered Icelandic tholeiites at temperatures in excess of $200^{\circ} \mathrm{C}$ and actinolite above $280^{\circ} \mathrm{C}$; in the same rocks epidote first occurs at temperatures of $260^{\circ} \mathrm{C}$ (Kristmannsdottir, 1978). The absence of epidote from amphibole-bearing assemblages at Site 555 is puzzling especially since calcite is also absent, but is clearly linked with a notable lack of plagioclase alteration. It may be that amphibole, which is invariably epitaxial on pyroxene, is a metastable phase, and that alteration temperatures at the bottom of Site 555 did not exceed $250^{\circ} \mathrm{C}$.

\section{DISCUSSION}

The basaltic rocks of Leg 81 vary little in petrography, mineralogy, and major and trace elements; they are subalkaline ("tholeiitic") and so fall in a major group of 
global basalts, whether of oceanic or continental occurrence. Texturally, the basalts of Sites 552, 553, 554, and 555 vary little, being mainly aphyric or weakly phyric but in places they become moderately phyric. The aphyric lithologies may represent their parent liquids and since in the phyric varieties microphenocrysts are mainly minor, these probably do not represent a significant departure from the parent liquid composition. The microphenocrysts are mainly labradorite to bytownite with sporadic clinopyroxene and sparse olivine, but they have not affected the major oxide chemistry, unlike phenocryst-rich basalts (or with dissolved major phenocrysts) which elsewhere would represent fractionated magma. The Leg 81 basalts are plagioclase-clinopyroxene-tholeiites formed by the early crystallization of plagioclase accompanied in some by clinopyroxene and sporadic traces of olivine, and second-phase cotectic crystallization of plagioclase and clinopyroxene with minor magnetite.

The only differences in chemistry are the slightly higher $\mathrm{CaO}$ contents of the olivine-dolerites of Site 555 and slightly higher $\mathrm{K}_{2} \mathrm{O}$ contents of the Site 554 basalts compared with the other basaltic rocks. But these differences are insignificant petrogenetically since the variation diagram (Alkali-SiO ${ }_{2}$, Fig. 2; FMA, Fig. 6) indicates that the basalts and dolerites of Leg 81 are consanguineous with a limited degree of evolution along the tholeiite trend.

Trace elements do not in general relate to specific basalt or dolerite textures, for example to the absence or presence of microphenocrysts, but we consider that some plagioclase "microphenocrysts" from Holes 552, 553A, and the uppermost basalts of Hole 555 represent xenocrysts fractionated from a more primitive source. Plots of $\mathrm{Cr} / \mathrm{Y}$ (Fig. 8) suggest that the lower $\mathrm{Cr}$ values from Holes 553A and 554A may be the result of crystal fractionation. Variation of $\mathrm{Cr}$ is essentially independent of Y, distinguishing the least-fractionated Hole 555 basaltic rocks from those of Holes 553A and 554A. Tuffs from Hole 555 are more fractionated than associated lavas, suggesting that the two did not have a common source.

Limited fractionation is also indicated by the immobile elements $\mathrm{Ti}, \mathrm{Zr}$, and Y (Fig. 10) and by the linear correlations noted in the SI plots (Fig. 3). The withinhole variations obscure any systematic fractionation with crystal development. The present basalt cores represent only the uppermost layers of what are probably very thick basaltic sequences. Evidence of magma mixing trend is obscured by the data spread, for example, in the plot of incompatible $\mathrm{Zr}$ against compatible $\mathrm{Ni}$, although a roughly negative linear correlation emerges. This suggests some normal fractionation in which $\mathrm{Zr}$ becomes concentrated in the residual liquid and $\mathrm{Ni}$ depleted, but the spread of our data indicates other factors such as mixing.

The nature of the parental liquid is accordingly very obscure, although the anorthite-rich $\left(\mathrm{An}_{86}\right)$ microphenocrysts in some feldspar-phyric basalts toward the base of Hole 553A indicate a more primitive source. The olivine-dolerite-basal unit of Hole 555 is chemically akin to, and passes up into, the overlying basalts in Sample 553A-95-1, 25-30 cm. The dolerite represents the massive, coarsely crystalline core of a flow unit of unknown total thickness and was comagmatic with the other basalts. The basalts of Hole 554A differ from the other sites only in higher $\mathrm{K}_{2} \mathrm{O}$, slightly higher $\mathrm{SiO}_{2}$ than Hole 555 basalts, and a pronounced positive Eu anomaly. These may indicate slightly greater degree of evolution than the other basalts, although the sampling and analytical factors involved may not support this. There is no enrichment in feldspar, for example, to explain the Eu anomaly.

The plot of $\mathrm{Ti} / 100: \mathrm{Zr}: \mathrm{Y} \times 3$ (Fig. 10) attempts to characterize the tectonic setting of the basaltic rocks of Leg 81. Taking due note of the reservations on the use of such discrimination diagrams (Wood et al., 1979; Prestvik, 1982), there is a small but perhaps significant intersite variation. Hole 553A and Hole 554A basalts group about the calc-alkali basalt boundary, whereas Hole 555 basic rocks concentrate in the ocean-floor basalt field. Tuffs plot in the same basaltic fields indicating the relative immobility of $\mathrm{Ti}$ in particular.

The basic rocks examined from Leg 81 match in petrography, mineralogy, and chemistry the general run of ocean-floor subalkaline ("tholeiitic") basalts. We can offer no firm evidence on the nature of the parental magma except that it probably underwent slight fractionation from more basic sources. Low-pressure crystal-melt equilibria existed during the lava flow eruptions and with cotectic precipitation of essentially plagioclasepyroxene but with xenocrystic incorporation from more basic material. So far as the present evidence shows, the first-formed ocean basalts of Hole 554 are in most respects consanguineous with those of the dipping reflectors.

Many of the basalts have suffered alteration through argillization of primary minerals. Since vesicles are commonly filled with smectite and celadonite (the two principal clay minerals) alteration probably accompanied or soon followed eruption of the flows, with devitrification and alteration of primary glass both in vesicles and in the mesostasis. Alteration was probably accelerated through autometasomatism or hydrothermal alteration with circulating fluids in the lava flows. Although primary mineralogy and the chemistry of the basalts do not provide direct evidence of the conditions of eruption, some indications of the postmagmatic environment are contained in secondary alteration products. The most positive evidence of subaerial eruption of part of the basalt sequence is found in Holes 553A and 555 where vitroclastic tuffs and agglomerates have been hematitized. While the latter may be caused by hydrothermal activity, hematite replacement of glass clasts in these tuffs preceded argillization of the glass margins and matrix, the latter containing typical seawater-alteration products. Some basalt flows in Hole 553A (as at Sections 43-1, 48-2 to 48-4, and 52-3) may also be altered subaerially, but it is difficult to distinguish such hematitization from that possibly associated with seawater alteration. Early subaerial alteration may also be inferred from the atypical phyllosilicate assemblage occur- 
ring at the top of Subunit Vc in Hole 553A, the presence of nontronite in particular demanding strongly oxidative alteration conditions.

Whatever the duration of subaerial weathering of the basalt at various site horizons, it seems certain that this was followed by prolonged and pervasive contact with seawater. However, the variety of secondary mineral assemblages found in basalts at different sites suggests widely different geothermal gradients in the two tectonically contrasted parts of Rockall Plateau drilled by Leg 81. At sites on the margin of the microcontinent (Holes $552,553 \mathrm{~A}$ and $554 \mathrm{~A}$ ), alteration has resulted from interaction between seawater and warm rock at temperatures below $200^{\circ} \mathrm{C}$ and most likely less than $150^{\circ} \mathrm{C}$; the sequence of minerals probably formed in response to falling temperatures. At Site 555, situated on Rockall Plateau, temperatures of $250-300^{\circ} \mathrm{C}$ may have been attained during greenschist-facies alteration of the deepest rocks penetrated. The depth to the greenschist zone from the original water/rock interface infers a maximum geothermal gradient of $\sim 1000^{\circ} \mathrm{C} / \mathrm{km}$, and a minimum one of $\sim 300^{\circ} \mathrm{C} / \mathrm{km}$, if it is assumed that the topmost flows were at a temperature of $\sim 100^{\circ} \mathrm{C}$. Geothermal gradients within this range are most unlikely to reflect regional gradients, and it seems likely therefore that at no great depth beneath Site 555 intrusive rocks underlie the lowest dolerites sampled.

\section{ACKNOWLEDGMENTS}

The authors wish to thank, in addition to the contributors named, Dr. M. T. Styles for invaluable assistance with electron microprobe analyses and computer programming, Mr. G. E. Strong for assistance with processing the trace-element data, and Mr. R. D. Fakes for the many thin sections. This contribution is published with permission of the Director, British Geological Survey (N.E.R.C.).

\section{REFERENCES}

Andrews, A. J., 1980. Saponite and celadonite in layer 2 basalts, DSDP Leg 37. Contrib. Mineral. Petrol., 73:323-340.

Bollingberg, H., Brooks, C. K., and Noe-Nygaard, A., 1975. Trace element variations in Faeroese basalts and their possible relationships to ocean floor spreading history. Bull. Geol. Soc. Den., 24: $55-60$.

Brooks, C. K., Nielson, T. F. D., and Petersen, T. S., 1976. The Blosseville Coast basalts of East Greenland: their occurrence, composition and temporal variations. Contrib. Mineral. Petrol., 58:279-292.

Bryan, W. B., Thompson, G., Frey, F. A., and Dickey, J. S., 1976. Inferred geologic settings and differentiation in basalts from the Deep Sea Drilling Project. J. Geophys. Res., 81:4285-4304.

Bryan, W. B., Thompson, G., and Ludden, J. N., 1981. Compositional variation in normal MORB from $22^{\circ}-25^{\circ} \mathrm{N}$ : Mid-Atlantic Ridge and Kane Fracture Zone. J. Geophys. Res., 86:11835-11836.

Buckley, H. A., Bevan, J. C., Brown, K. M., Johnson, L. R., and Farmer, V. C., 1978. Glauconite and celadonite: two separate mineral species. Mineral. Mag., 42:373-382.

Cann, J. R., 1971. Major element variations in ocean-floor basalts. Philos. Trans. R. Soc. London Ser. A, A268:495-505.

Emmermann, R., and Puchett, H. 1979. Abundances of rare earths and other trace elements in Leg 46 Basalts (DSDP). In Dmitriev, L., Heirtzler, J., et al., Init. Repts. DSDP, 46: Washington (U.S. Govt. Printing Office), 241-246.
Erlank, A. J., and Kable, E. J. D., 1976. The significance of incompatible elements in Mid-Atlantic Ridge basalts from $45^{\circ} \mathrm{N}$ with particular reference to $\mathrm{Zr} / \mathrm{Nb}$. Contrib. Mineral. Petrol., 54:281-291.

Frey, F. A., Bryan, W. B., and Thompson, G., 1974. Atlantic Ocean floor: geochemistry and petrology of basalts from Legs 2 and 3 of the Deep Sea Drilling Project. J. Geophys. Res., 79:5507-5527.

Frey, F. A., Haskin, M. A., Poetz, J. A., and Haskin, L. A., 1968. Rare-earth abundances in some basic rocks. J. Geophys. Res., 73: 6085-6097.

Haskin, L. A., 1979. On rare-earth element behaviour in igneous rocks. In Ahrens, L. H. (Ed.), Origin and Distribution of the Elements, Physics and Chemistry of the Earth (Vol. 11): London (Pergamon), pp. 175-189. (2nd Symposium on the Origin and Distribution of the Elements, Paris, 1977.)

Haskin, L. A., Haskin, M. A., Frey, F. A., and Wildeman, T. R., 1968. Relative and absolute terrestrial abundances of the rare earths. In L. H. Ahrens (Ed.), Origin and Distribution of the Elements: London (Pergamon), pp. 890-911. (Symposium on the Origin and Distribution of the Elements, Paris, 1967)

International Union of Geological Sciences, 1981. Subcommission on the Systematics of Igneous Rocks. Minutes of the Cambridge Working Meeting, 21-24 September, 1981 (unpublished).

Irvine, T. N., and Baragar, W. R. A., 1971. A guide to the chemical classification of the common volcanic rocks. Can. J. Earth Sci., 8: 523-548.

Kristmannsdottir, H., 1978. Alteration of basaltic rocks by hydrothermal activity at $100-300^{\circ} \mathrm{C}$. In Mortland, M. M., and Farmer, V. C. (Eds.), Developments in Sedimentology (Vol. 27): New York (Elsevier). (International Clay Conference, 1978).

Kuno, H., 1960. High-alumina basalt. J. Petrol., 121-145.

Laughton, A. S., Berggren, W. A., et al., 1972. Init. Repts. DSDP, 12: Washington (U.S. Govt. Printing Office).

Luyendyk, B. P., Cann, J. R., et al., 1979. Init. Repts. DSDP, 49: Washington (U.S. Govt. Printing Office).

Miyashiro, A., Shido, F., and Ewing, M. 1971. Metamorphism in the Mid-Atlantic Ridge near $24^{\circ}$ and $30^{\circ} \mathrm{N}$. Philos. Trans. R. Soc. London, Ser. A, 268:589-603.

Nicholls, G. D., and Islam, M. R., 1971. Geochemical investigations of basalts and associated rocks from the ocean floor and their implications. Philos. Trans. R. Soc. London, Ser. A., 268:469-486.

Nockolds, S. R., 1954. Average chemical compositions of some igneous rocks. Geol. Soc. Am. Bull., 65:1021.

Nockolds, S. R., Knox, R. W. O'B., and Chinner, G. A., 1978. Petrology for Students: London (Cambridge University Press).

Pearce, J. A., and Cann, J. R., 1973. Tectonic setting of basic volcanic rocks determined using trace element analysis. Earth Planet. Sci. Lett., 19:290-300.

Prestvik, T., 1982. Basic volcanic rocks and tectonic setting. A discussion of the $\mathrm{Zr}-\mathrm{Ti}-\mathrm{Y}$ discrimination diagram and its suitability for classification purposes. Lithos, 15:241-247.

Rankama, K., and Sahama, Th. G., 1950. Geochemistry: Chicago (The University of Chicago Press).

Roex, A. P. le, Erlank, A. J., and Needham, H. D., 1981. Geochemical and mineralogical evidence for the occurrence of at least three magma types in the 'Famous' Region. Contrib. Mineral. Petrol., 77:24-37.

White, W. M., and Schilling, J.-G., 1978. The nature and origin of geochemical variation in Mid-Atlantic Ridge basalts from the Central North Atlantic. Geochim. Cosmochim. Acta, 42:1501-1516.

Wood, D. A., Varet, J., Bougault, H., Corre, O., Joron, J. L., Treuil, M., Bizouard, H., Norry, M. J., Hawkesworth, C. J. and Roddick, J. C., 1979. The petrology, geochemistry and mineralogy of North Atlantic basalts, a discussion based on IPOD Leg 49. In Luyendyk, B. P., Cann, J. R., et al., Init. Repts. DSDP, 49: Washington (U.S. Govt. Printing Office), 597-655.

Date of Acceptance: April 21, 1983 


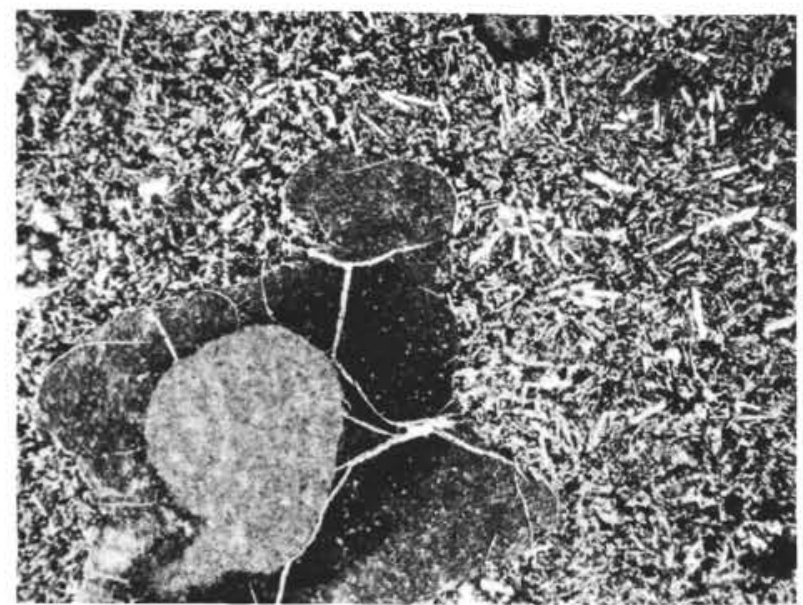

1

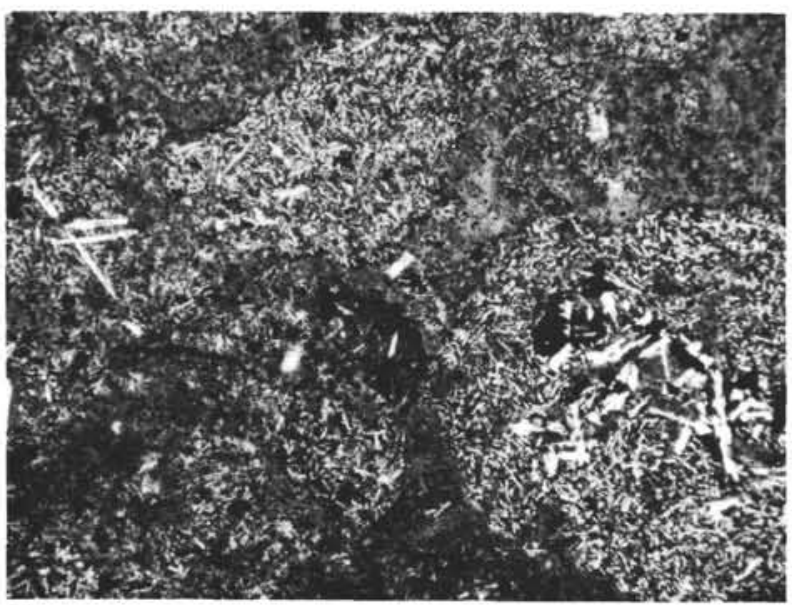

3

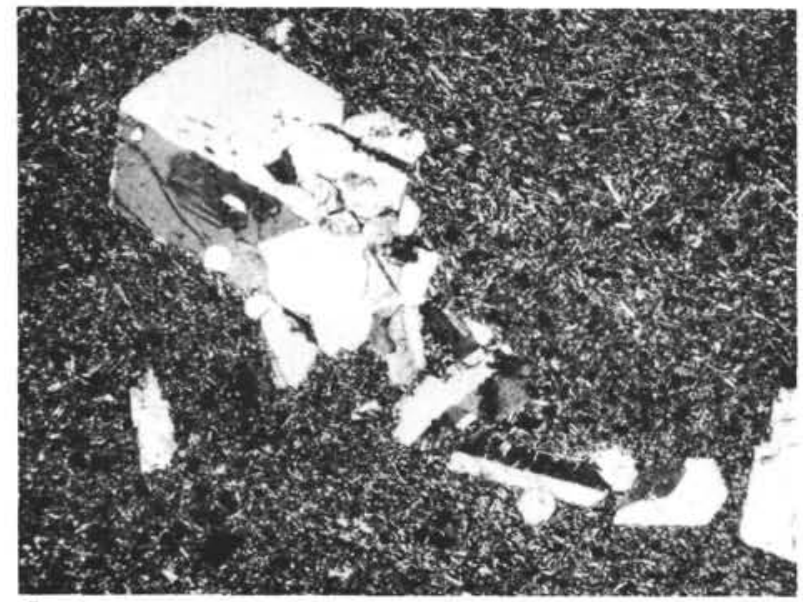

5
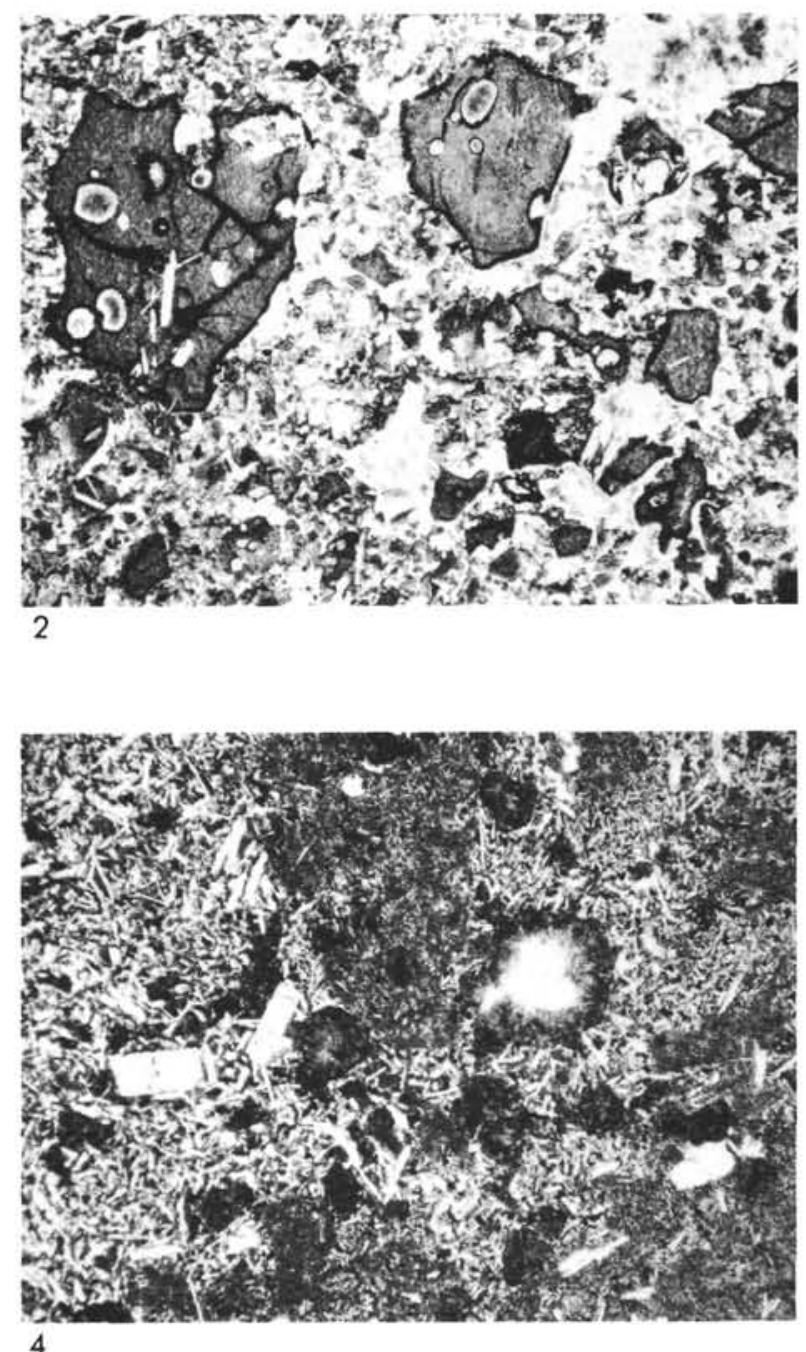

4

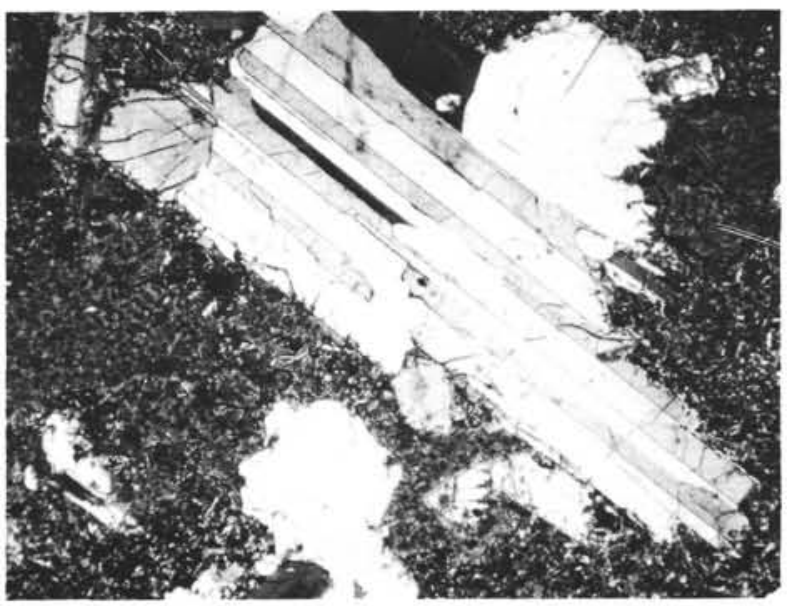

6

Plate 1. Photomicrographs of igneous rocks from the "dipping reflectors" from DSDP Leg 81, Hole 553A (Figs. 5 and 6 were taken under crossed polarizers). 1. Amygdaloidal basalt from near top of flow unit showing vesicles infilled with celadonite (center) and smectite (dark, outer), with an aphyric groundmass felt of plagioclase laths and altered interstices; Sample 553A-39-1, 2-4 cm (magnification 28 $\times$ ). 2. Hyaloclastite showing angular fresh pumiceous glass particles in a fine-grained vitroclastic matrix; Sample 553A-45-1, 87-91 cm (magnification $28 \times$ ). 3. Basaltic autobreccia (flow breccia?) showing patches of plagioclase-microphyric basalt in altered glassy or aphyric basalt; Sample 553A-45-4, 16-20 cm (magnification $28 \times$ ). 4. Plagioclase-microphyric basalt complexly associated with aphyric basalt from base of flow unit; Sample 553A-47-4, $81-84 \mathrm{~cm}$ (magnification $28 \times$ ). 5. Plagioclase-cumulophyric basalt from middle of flow unit with euhedral labradorite $\left(\mathrm{An}_{50}\right)$ up to $1.8 \mathrm{~mm}$ long set in a microcrystalline felted groundmass; Sample 553A-51-1, 54-57 cm (magnification $28 \times$ ). 6. Plagioclase-macrophyic basalt showing prominent labradorite $\left(\mathrm{An}_{60}\right)$ prisms in a microcrystalline felted groundmass; Sample 553A-59-4, 93-95 cm (magnification $28 \times$ ). 

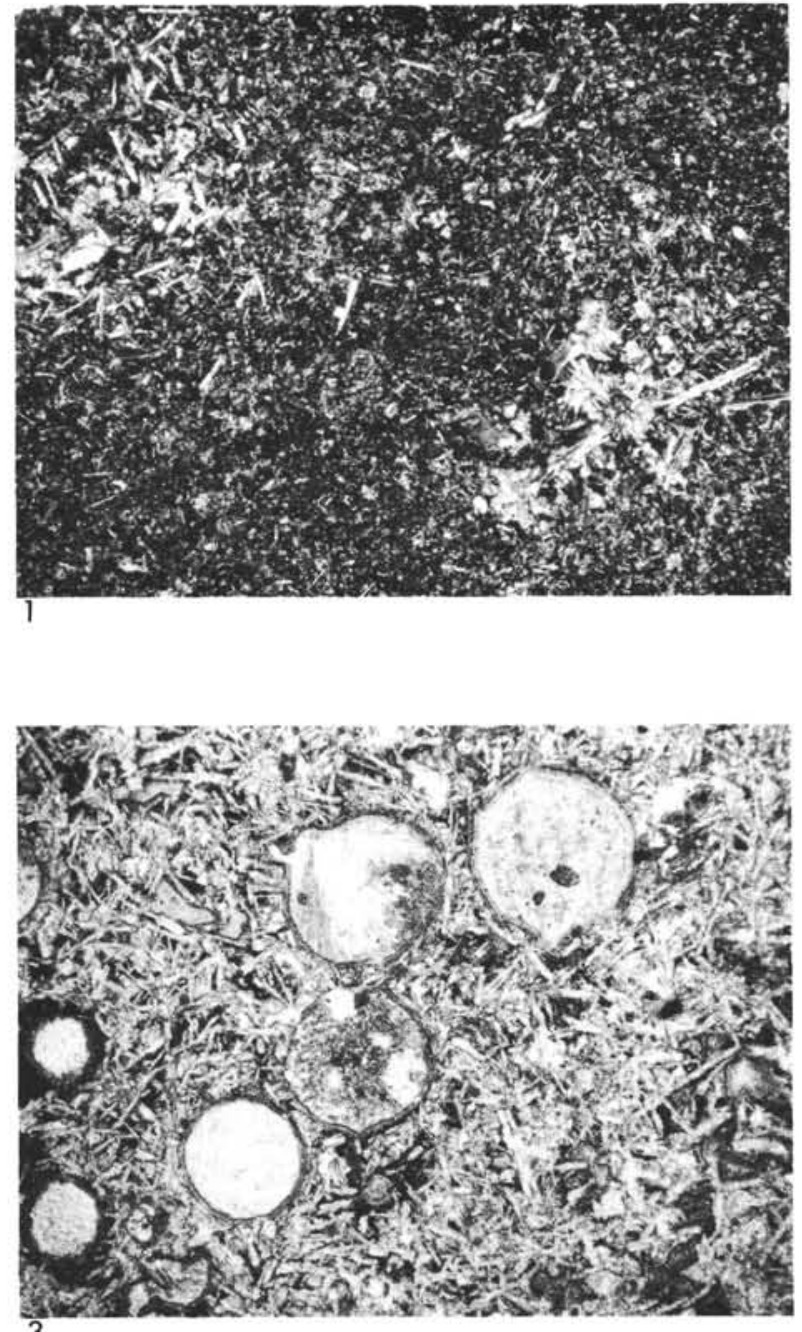

3

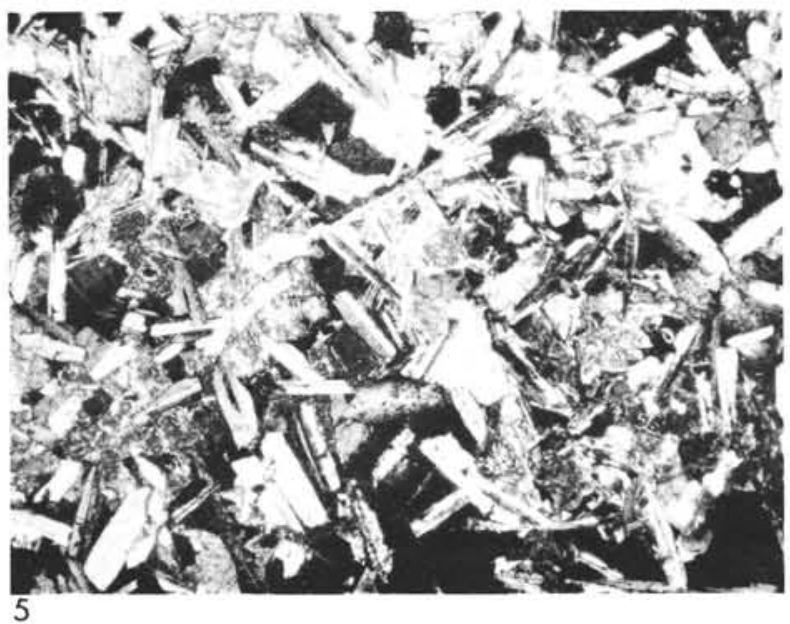

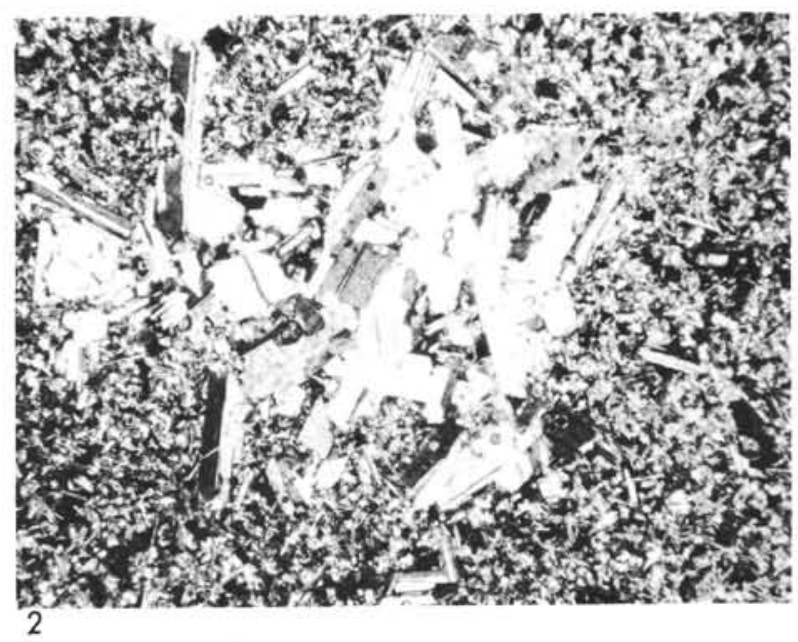
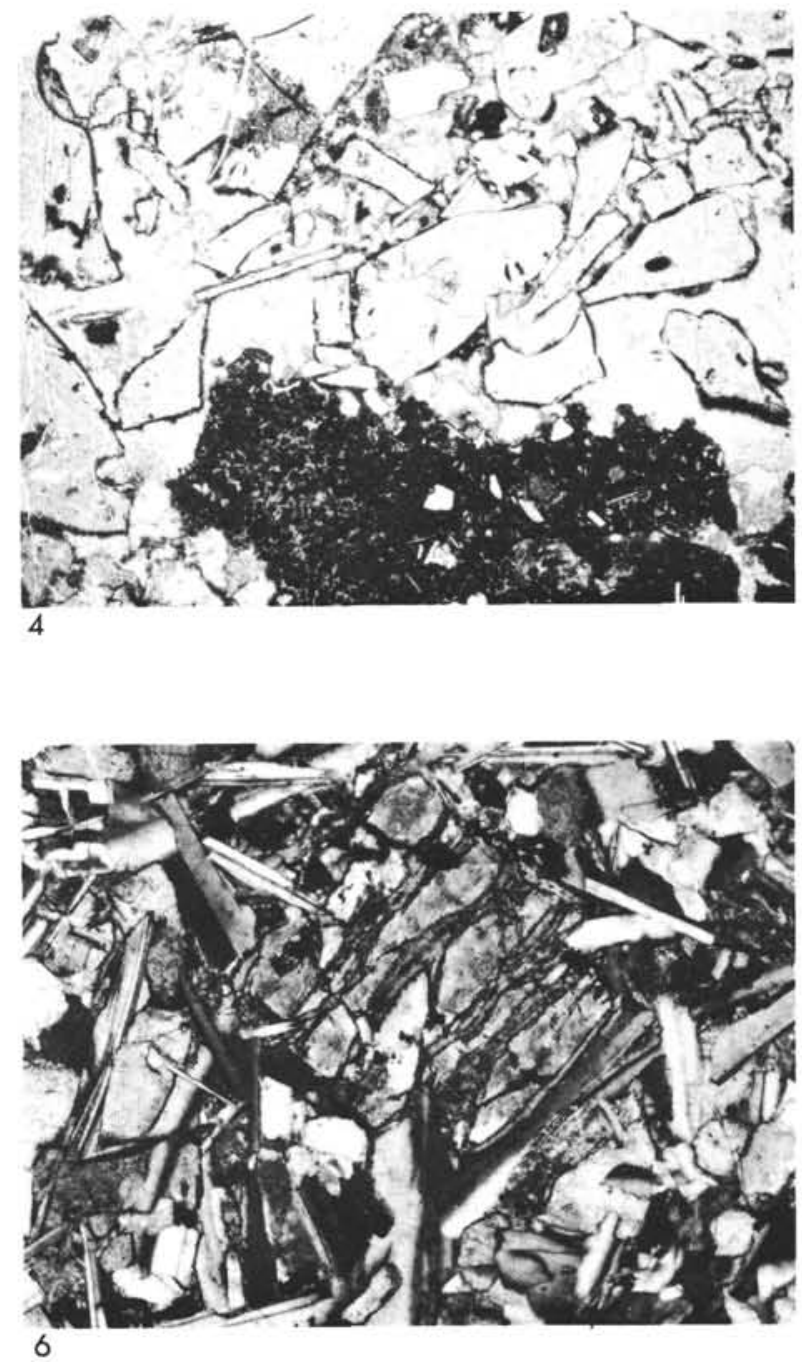

Plate 2. Photomicrographs of basement igneous rocks from the crest of the "outer high" (Site 544) and from midway between the Hatton and

Edoras banks (Site 555) DSDP Leg 81. (Figs. 1, 2, 5, and 6 were taken under crossed polarizers). 1. Plagioclase-microphyric basalt showing patches of coarser, very slender labradorite laths set in a microcrystalline ground of plagioclase and pyroxene granules. Sample 554A-14-1, 36-39 $\mathrm{cm}$ (magnification $28 \times$ ) 2 2. Plagioclase-cumulophyric basalt with a prominent cluster of labradorite laths $\left(\mathrm{An}_{56}\right)$ in a microcrystalline felted groundmass. Sample 555-69-3,113-116 cm (magnification $28 \times$ ). 3. Amygdaloidal basalt with abundant spheroidal amygdules containing quartz and smectite; the base is a fine felt of labradorite laths and intergranular pyroxene, partly altered to smectite; Sample 555-76-5, 22-24 cm (magnification $28 \times$ ). 4. Hyaloclastite composed of very angular glass fragments and microphyric basalt; Sample 555-77-4, 74-77 cm (magnification $28 \times$ ) 5. Ophitic olivine-dolerite with a framework of labradorite laths and partly intergrown clinopyroxene; Sample 555-83-1, 42-45 cm (magnification $28 \times$ ). 6. Olivine-dolerite showing fresh olivine (center) subophitically intergrown with labradorite laths $\left(\mathrm{An}_{54}\right)$; $\mathrm{Sample}$ $555-95-1,97-100 \mathrm{~cm}$ (magnification $100 \times$ ). 